\title{
5d and 4d SCFTs: canonical singularities, trinions and S-dualities
}

\author{
Cyril Closset, ${ }^{b, a}$ Simone Giacomelli, ${ }^{a}$ Sakura Schäfer-Nameki ${ }^{a}$ and Yi-Nan Wang ${ }^{a}$ \\ ${ }^{a}$ Mathematical Institute, University of Oxford, \\ Andrew-Wiles Building, Woodstock Road, Oxford OX2 6GG, U.K. \\ ${ }^{b}$ School of Mathematics, University of Birmingham, \\ Watson Building, Edgbaston, Birmingham B15 2TT, U.K. \\ E-mail: c.closset@bham.ac.uk
}

ABstract: Canonical threefold singularities in M-theory and Type IIB string theory give rise to superconformal field theories (SCFTs) in $5 \mathrm{~d}$ and $4 \mathrm{~d}$, respectively. In this paper, we study canonical hypersurface singularities whose resolutions contain residual terminal singularities and/or 3-cycles. We focus on a certain class of 'trinion' singularities which exhibit these properties. In Type IIB, they give rise to $4 \mathrm{~d} \mathcal{N}=2$ SCFTs that we call $D_{p}^{b}(G)$-trinions, which are marginal gaugings of three SCFTs with $G$ flavor symmetry. In order to understand the $5 \mathrm{~d}$ physics of these trinion singularities in M-theory, we reduce these $4 \mathrm{~d}$ and $5 \mathrm{~d}$ SCFTs to $3 \mathrm{~d} \mathcal{N}=4$ theories, thus determining the electric and magnetic quivers (or, more generally, quiverines). In M-theory, residual terminal singularities give rise to free sectors of massless hypermultiplets, which often are discretely gauged. These free sectors appear as 'ugly' components of the magnetic quiver of the 5d SCFT. The 3 -cycles in the crepant resolution also give rise to free hypermultiplets, but their physics is more subtle, and their presence renders the magnetic quiver 'bad'. We propose a way to redeem the badness of these quivers using a class $\mathcal{S}$ realization. We also discover new S-dualities between different $D_{p}^{b}(G)$-trinions. For instance, a certain $E_{8}$ gauging of the $E_{8}$ Minahan-Nemeschansky theory is S-dual to an $E_{8}$-shaped Lagrangian quiver SCFT.

Keywords: Field Theories in Higher Dimensions, String Duality, Supersymmetric Gauge Theory, Differential and Algebraic Geometry

ArXiv EPRINT: 2012.12827 


\section{Contents}

1 Introduction and overview 1

1.1 Terminal singularities, hypermultiplets and discrete gauging 3

1.2 Higgs branches for 5d SCFTs from quiver(ine)s 4

1.3 Trinion singularities and $D_{p}^{b}(\mathrm{SU}(N))$ SCFTs $\quad 6$

$\begin{array}{lll}1.4 & \text { Trinions,class } \mathcal{S} \text { theories and generalized toric polygons } & 7\end{array}$

1.5 Trinions of type $G=D, E$ and new S-dualities 8

$24 \mathrm{~d} \mathcal{N}=2$ SCFTs $D_{p}^{b}(G)$, trinion singularities, and 3d reduction 9

2.1 Canonical singularities and 4d SCFTs 9

$\begin{array}{ll}2.2 D_{p}^{b}(G) \text { : the executive summary } & 10\end{array}$

2.3 Reduction to Lagrangian theories for $G=\mathrm{SU}(N) \quad 12$

2.3.1 Dimensionally-reduced $D_{2}(\mathrm{SU}(N))$ theories $\quad 13$

$\begin{array}{lll}2.3 .2 & D_{p}^{b}(\mathrm{SU}(N)) \text { theories and 3d quivers } & 14\end{array}$

$\begin{array}{lll}2.3 .3 & \text { Examples } & 18\end{array}$

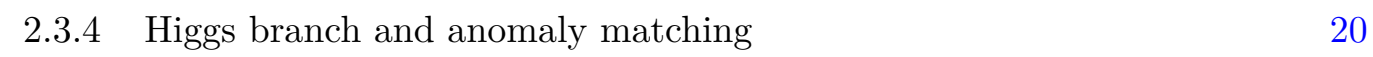

2.4 Quiverines from marginally-gauged $D_{p}^{b}(\mathrm{SU}(N))$-trinions 21

$\begin{array}{lll}2.4 .1 & \text { Trinion singularities } & 21\end{array}$

$\begin{array}{lll}2.4 .2 & \text { The } 3 \text { d reduction of } \mathscr{T}_{X}^{4 \mathrm{~d}} & 22\end{array}$

3 Terminal singularities and rank 0 5d SCFTs $\quad 23$

$3.1 \mathscr{T}_{X}^{4 \mathrm{~d}}$ and trinion-SCFTs for rank $0 E_{n}^{(k)}$ theories 23

$\begin{array}{ll}3.2 & \text { Magnetic quivers and higher form symmetries } \\ \end{array}$

$\begin{array}{lll}3.2 .1 & \text { The } E_{6} \text { model } & 26\end{array}$

$\begin{array}{ll}3.2 .2 & \text { The } E_{7} \text { models }\end{array}$

$\begin{array}{lll}3.2 .3 & \text { The } E_{8} \text { models } & 28\end{array}$

4 Generalized $\boldsymbol{E}_{\boldsymbol{n}}$ theories and discrete gauging $\quad 30$

4.1 The rank-one $E_{n}^{(k)}$ theories and their magnetic quivers 31

4.1.1 The $r=1 E_{6}^{(k)}$ theories $\quad 32$

4.1.2 The $r=1 E_{7}^{(k)}$ and $E_{8}^{(k)}$ theories $\quad 35$

4.2 The higher-rank $E_{n}^{(k)}$ theories 36

5 Class $\mathcal{S}$ description, brane-webs and partial higgsing $\quad 40$

5.1 Rank-zero trinions 41

5.2 Coulomb branch spectrum and 'badness' in the class $\mathcal{S}$ description $\quad 42$

5.3 Redeeming bad theories by box-lifting 44

5.3.1 Rank-one $E_{n}^{(k)}$ theories and GTPs 44

$\begin{array}{lll}\text { 5.3.2 Higher-rank theories } & 47\end{array}$ 
6 Classification of the $\mathrm{SU}(N)$ trinions $\quad 48$

6.1 Case $\left(b_{\alpha}\right)=(N, N, N) \quad 49$

$\begin{array}{lll}6.2 & \text { Case }\left(b_{\alpha}\right)=(N, N, N-1) & 50\end{array}$

6.3 Case $\left(b_{\alpha}\right)=(N, N-1, N-1)$

6.4 Case $\left(b_{\alpha}\right)=(N-1, N-1, N-1) \quad 55$

$7 \quad D_{p}^{b}(G)$-trinions SCFTs for $G=D, E \quad 56$

7.1 Trinion geometries for generic $G \quad 56$

$\begin{array}{ll}7.2 D_{p}^{b}(G) \text {-trinions with } b_{1}=h^{\vee} \text { and } p_{1}=2 & 57\end{array}$

$\begin{array}{lll}7.2 .1 & b_{2}=b_{3}=h^{\vee} & 57\end{array}$

$\begin{array}{ll}7.2 .2 & \text { Other sporadic models }\end{array}$

$7.3 D_{p}^{b}(G)$-trinions with $b_{1} \neq h^{\vee}$ and $p_{1}=1 \quad 58$

7.4 Matching the CB spectrum to the spectrum of the singularity 58

$8 \quad$ S-dualities between $4 \mathrm{~d} D_{p}^{b}(G)$-trinions $\quad 59$

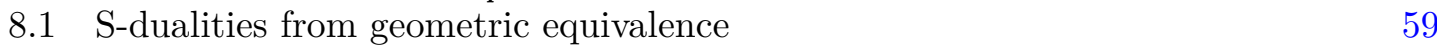

8.2 A list of new S-dualities 60

A Resolutions of hypersurface canonical singularities $\quad 63$

A.1 $E_{n}^{(k)}$ theories 63

A.2 13 sporadic trinion models $\quad 65$

$\begin{array}{lll}\text { A.3 Type } \mathrm{VIII}_{2}(N, N, 2,2) \text { series } & 69\end{array}$

$\begin{array}{ll}\text { B Proof of the completeness of the } \mathrm{SU}(N) \text { trinions } & 70\end{array}$

C Tables of trinions with $G=D, E \quad 72$

\section{Introduction and overview}

Five-dimensional superconformal field theories (5d SCFTs) are intrinsically strongly-coupled. Upon mass deformations, they are often related to $5 \mathrm{~d} \mathcal{N}=1$ gauge theories, that arise as IR-free low-energy effective descriptions. In this work, we consider $5 \mathrm{~d}$ SCFTs $\mathcal{T}_{\mathbf{X}}^{5 \mathrm{~d}}$ constructed as the low-energy description of M-theory on $\mathbb{R}^{1,4} \times \mathbf{X}$, where $\mathbf{X}$ is a canonical threefold singularity. This approach was initiated in [1-4] and further studied e.g. in [535], revealing a rich landscape of 5d SCFTs. One of the challenges, in this approach, is to identify the precise map between the canonical threefold singularities and the 5 d physics. This is best understood, when the geometry allows a complete Calabi-Yau resolution (i.e. a crepant resolution, which leaves the canonical class invariant). However, this vanilla situation is far from generic. In many instances, the canonical singularity after resolution still has remnant singularities, so-called terminal singularities, which cannot be crepantly resolved. It is the physics of such canonical singularities, and the study of their Higgs and extended Coulomb branches, that are the subject of this paper. 
The $5 \mathrm{~d} \mathcal{N}=1$ gauge-theory description, when it exists, corresponds to a (partial) crepant resolution of the singularity $[2,4]$

$$
\pi: \widetilde{\mathbf{X}} \rightarrow \mathbf{X} \text {. }
$$

By definition, a crepant resolution satisfies $K_{\widetilde{\mathbf{X}}}=\pi^{*} K_{\mathbf{X}}$. Following [36, 37], we choose $\mathbf{X}$ to be a quasi-homogeneous hypersurface singularity in $\mathbb{C}^{4}$

$$
\mathbf{X} \cong\left\{\left(x_{1}, x_{2}, x_{3}, x_{4}\right) \subset \mathbb{C}^{4} \mid F\left(x_{1}, x_{2}, x_{3}, x_{4}\right)=0\right\},
$$

with an isolated singularity at the origin. A crepant resolution (1.1) corresponds to probing the extended Coulomb branch (ECB) of $\mathcal{T}_{\mathbf{X}}^{5 \mathrm{~d}}$, while a generic deformation of the singularity, denoted by $\widehat{\mathbf{X}}$, corresponds to probing the quantum Higgs branch (HB).

The classical geometry of the crepant resolution (1.1) has a rich and interesting structure. It is partially characterized by three integers $r, f$ and $b_{3}$, which correspond to the number of compact cycles within the (partially) resolved threefold

$$
\operatorname{dim} H_{2}(\tilde{\mathbf{X}}, \mathbb{R})=r+f, \quad \operatorname{dim} H_{3}(\widetilde{\mathbf{X}}, \mathbb{R})=b_{3}, \quad \operatorname{dim} H_{4}(\widetilde{\mathbf{X}}, \mathbb{R})=r .
$$

In particular, $r$ is the number of exceptional divisors (i.e. compact 4-cycles), which encode the rank of the 5d SCFT $\mathcal{T}_{\mathbf{X}}^{5 \mathrm{~d}}$ - by definition, the real dimension of its Coulomb branch. In addition, $f$ is the number of curves (2-cycles calibrated by the Kähler form) dual to non-compact divisors, giving rise to a distinguished abelian symmetry $\mathrm{U}(1)^{f}$ of $\mathcal{T}_{\mathbf{X}}^{5 \mathrm{~d}}$ that survives on its CB. The dimension of the ECB is $r+f$, including $f$ real mass parameters. Furthermore, in the presence of non-trivial 3-cycles (that is, for $b_{3}>0$ ) in the resolution, we have additional free hypermultiplets (in their Higgsed phase) at the point of the CB corresponding to $\widetilde{\mathbf{X}}$. The integers $r, f$ and $b_{3}$ are the same for any crepant resolution of $\widetilde{\mathbf{X}}$ that results in a smooth geometry (see e.g. the general CB analysis in [6] and the mathematical analysis of [38]), and are therefore intrinsic to the canonical singularity.

The literature on the M-theory engineering of 5d SCFTs has mostly focussed on canonical singularities $\mathbf{X}$ for which the resolved singularity $\widetilde{\mathbf{X}}$ is completely smooth, with its exceptional locus $\pi^{-1}(0)$ consisting of a union of smooth surfaces, and with $b_{3}=0$. In this case, $f$ gives the rank of the flavor symmetry $G_{\mathrm{H}}^{5 \mathrm{~d}}$ of $\mathcal{T}_{\mathbf{X}}^{5 \mathrm{~d}}$. Interestingly, this is not the generic situation, since a maximal crepant resolution is generally not entirely smooth. ${ }^{1}$ Various interesting phenomena can occur, including the following two:

- The threefold $\tilde{\mathbf{X}}$ has $b_{3}>0$. This happens in the presence of exceptional divisors that are ruled surfaces over curves of genus greater than $1[36,38]$.

- The threefold $\widetilde{\mathbf{X}}$ is not fully resolvable, i.e. it contains residual isolated terminal singularities.

These two phenomena can co-exist and are logically independent. In this work, we explore them in a number of examples, which are under particularly good control, following the

\footnotetext{
${ }^{1}$ In $6 \mathrm{~d}$ SCFTs engineered from elliptic threefolds in F-theory, these effects have been studied in [39, 40], while in $5 \mathrm{~d}$ and $4 \mathrm{~d}$ SCFTs this was discussed in [36].
} 
general logic advocated in [36, 37]. This allows us to bypass the difficulties in interpreting terminal singularities directly in M-theory. We find that these terminal singularities are associated to free sectors in $\mathcal{T}_{\mathbf{X}}^{5 \mathrm{~d}}$, modulo some discrete gauging. Along the way, we also obtain various new results about certain $4 \mathrm{~d} \mathcal{N}=2$ SCFTs and their $3 \mathrm{~d}$ reductions, as we will explain momentarily.

\subsection{Terminal singularities, hypermultiplets and discrete gauging}

By definition, a terminal singularity is a canonical singularity that admits a resolution with exceptional divisors $S_{i}$ such that

$$
K_{\widetilde{\mathbf{x}}}=\pi^{*} K_{\mathbf{X}}+\sum a_{i} S_{i}, \quad \text { with } \quad a_{i}>0, \quad \text { for all } i,
$$

as opposed to $a_{i}=0$ for all $i$ in the case of a crepant resolution. It thus correspond to $r=0$, giving us a 'rank-zero' $5 \mathrm{~d}$ theory $\mathcal{T}_{\mathbf{X}}^{5 \mathrm{~d}}$. If, in addition, we have $f=0$, the singularity does not admit any small resolution. More generally, terminal singularities sometimes admit (partial) small resolutions, in which case the exceptional locus of $\widetilde{\mathbf{X}}$ is a union of $f$ curves. For $r>0$, the singularity allows for $r$ many crepant blowups, however there can be a remnant terminal singularity, i.e. (1.4) holds true for $a_{i}=0$ for $i=1, \cdots, r$, and the remaining $a_{i}>0$. In either case, we would like to understand these $5 \mathrm{~d}$ theories more systematically.

The simplest example of a terminal singularity with $f>0$ is the conifold, $\mathbf{X} \cong\left\{x_{1}^{2}+\right.$ $\left.x_{2}^{2}+x_{3}^{2}+x_{4}^{2}=0\right\}$, which has $f=1$. The corresponding $5 \mathrm{~d}$ theory is the free massless hypermultiplet. Since the resolved conifold is smooth, the usual tools are applicable. The theory is gapped, corresponding to a massive hypermultiplet, with the massive BPS particle realized by the M2-brane wrapped on the exceptional curve.

A simple example of a terminal singularity with $f=0$ is the so-called $A_{2}$ singularity $\mathbf{X} \cong\left\{x_{1}^{2}+x_{2}^{2}+x_{3}^{2}+x_{4}^{3}=0\right\}$. It was recently argued that this singularity engineers a single hypermultiplet as well [36]. In this case, however, we do not know how to see this directly in M-theory. Instead, the conclusion follows from a chain of string theory and field theory dualities, from which we deduce that the Higgs branch of $\mathcal{T}_{\mathbf{X}}^{5 \mathrm{~d}}$ is $\mathbb{H}$, the same as for a single hypermultiplet.

It is tempting to conjecture that terminal singularities, however complicated, always correspond to $5 \mathrm{~d}$ hypermultiplets, perhaps up to a decoupled topological sector, or discrete gauging. One of our aims, in this paper, is to substantiate that expectation. For the theories and geometries studied in this paper, we make the following

Observation 1: for $\mathbf{X}$ a terminal singularity, the Higgs branch of the rank-zero theory $\mathcal{T}_{\mathbf{X}}^{5 \mathrm{~d}}$ is always given by $\mathbb{H}^{d_{\mathrm{H}}}$ or a discrete gauging thereof, where $\mathbb{H} \cong \mathbb{C}^{2}$ denotes the quaternionic plane

$$
\mathrm{HB}\left[\mathcal{T}_{\mathrm{X}}^{5 \mathrm{~d}}\right] \cong \mathbb{H}^{d_{\mathrm{H}}} \quad \text { or } \quad \mathbb{H}^{d_{\mathrm{H}} / \mathfrak{f}}
$$

For hypersurface singularities, the number of hypermultiplets is given by

$$
d_{\mathrm{H}}=\frac{\mu+f}{2},
$$


where $\mu$ is the Milnor number of $\mathbf{X}$. The discrete gauge group $\mathfrak{f}$ is determined by the singularity itself [36]. Depending on a choice of global structure, we can either have the free theory with a distinguished 0 -form symmetry $\mathfrak{f}$, or we can gauge that symmetry to obtain the quotient $\mathbb{H}^{d_{\mathrm{H}}} / \mathfrak{f}$. We will determine this quotient in a number of examples.

We would naturally like to conjecture that the same is true for any terminal singularity, but we leave a more detailed analysis for future work.

More generally, we would like to understand the situation wherein we have residual terminal singularities upon resolving a larger canonical singularity. We will see that, in a number of examples, the terminal singularities simply contribute additional free sectors, up to some discrete gauge group that now acts on the interacting sector as well. This leads us to the second observation, which we expect to be true more generally:

Observation 2: for $\mathrm{X}$ a canonical singularity with remnant terminal singularities once it is resolved, the associated rank 0 sector of the theory is a free sector, modulo discrete gauging, i.e. the $5 \mathrm{~d}$ theory is of the form $\left(\mathcal{T} \otimes \mathbb{H}^{k}\right) / \Gamma$, with $\Gamma$ a discrete group (which depends on the singularity and on a choice of global structure).

Before discussing the specific class of examples that we will study, let us first explain our general strategy [36]. In particular, let us explain how we determine the quantum Higgs branch of $\mathcal{T}_{\mathbf{X}}^{5 \mathrm{~d}}$.

\subsection{Higgs branches for 5d SCFTs from quiver(ine)s}

As alluded to above, deforming the singularity $\mathbf{X}$ into a smooth threefold $\widehat{\mathbf{X}}$ corresponds to probing the quantum HB of the $5 \mathrm{~d}$ SCFT $\mathcal{T}_{\mathbf{X}}^{5 \mathrm{~d}}$. The precise algebraic structure of the 5 d Higgs branch, let alone its hyperkähler metric, is hard to determine in the M-theory engineering, however, due in part to the presence of M2-brane instantons wrapping the compact 3 -cycles of the smooth threefold $\widehat{\mathbf{X}}$ (the deformed singularity). As emphasized in $[41,42]$, a fruitful avenue to study this system is to compactify the $5 \mathrm{~d}$ theory on a torus, in which case the $5 \mathrm{~d}$ HB can be recovered as the $3 \mathrm{~d} \mathcal{N}=4$ Coulomb branch of a so-called magnetic quiver(ine), denoted by $\mathrm{MQ}^{(5)}$, so that

$$
\mathrm{HB}\left[\mathcal{T}_{\mathbf{X}}^{5 \mathrm{~d}}\right] \cong \mathrm{CB}\left[\mathrm{MQ}^{(5)}\right]
$$

These theories $\mathrm{MQ}^{(5)}$ can sometimes be described as quiver gauge theories in $3 \mathrm{~d}$, but they are generally non-Lagrangian in our geometric setup, in which case we call them 'quiverines' [36]. The $3 \mathrm{~d} \mathcal{N}=4$ theory $\mathrm{MQ}^{(5)}$ is closely related to the circle reduction of a $4 \mathrm{~d} \mathcal{N}=2$ SCFT, denoted by $\mathscr{T}_{\mathbf{X}}^{4 \mathrm{~d}}$, which arises in Type IIB string theory compactified on the same canonical singularity. The electric quiverine $\mathrm{EQ}^{(4)}$ is the $3 \mathrm{~d} \mathcal{N}=4$ SCFT defined as the infrared (IR) limit of $\mathscr{T}_{\mathbf{X}}^{4 \mathrm{~d}}$ compactified on a circle, and the magnetic quiverine of $\mathcal{T}_{\mathbf{X}}^{5 \mathrm{~d}}$ is obtained by gauging a distinguished abelian flavor symmetry of $\mathrm{EQ}^{(4)}[36]$

$$
\mathrm{EQ}^{(4)} \stackrel{3 \mathrm{~d}}{\cong} D_{S^{1}} \mathscr{T}_{\mathbf{X}}^{4 \mathrm{~d}}, \quad \quad \mathrm{MQ}^{(5)} \cong \mathrm{EQ}^{(4)} / \mathrm{U}(1)^{f}
$$

This $3 \mathrm{~d}$ gauging is an 'S-operation' in the sense of [43]. Essentially by construction, $\mathrm{MQ}^{(5)}$ is the $3 \mathrm{~d} \mathcal{N}=4$ mirror of the 'electric quiverine' $\mathrm{EQ}^{(5)}$ defined as the IR limit of $\mathcal{T}_{\mathbf{X}}^{5 \mathrm{~d}}$ 


$$
5 \mathrm{~d} \mathcal{N}=1
$$



Figure 1. Summary of the relations between the supersymmetric QFTs in $5 \mathrm{~d}, 4 \mathrm{~d}$ and $3 \mathrm{~d}$ associated to a conical threefold singularity $\mathbf{X}$ in M-theory and type II string theory. The downward arrows denote dimensional reduction on circles. The $3 \mathrm{~d} \mathcal{N}=4$ theories are the 'quiverines', which are all related by $3 \mathrm{~d}$ mirror symmetry and by S-type gauging of $\mathrm{U}(1)^{f}$ flavor symmetries.

compactified on $T^{2} \cong S^{1} \times S_{\beta}^{1}$, where the first circle is the 'M-theory circle' that controls the Type-IIA string coupling. The general setup is summarized in figure 1. The relations between the $3 \mathrm{~d} \mathcal{N}=4$ quiverines essentially follows from T-duality of Type II string theory [44].

We use the neologism quiverine for an abstract $3 \mathrm{~d} \mathcal{N}=4$ (superconformal) field theory obtained from some higher-dimensional field theory upon circle compactification to $3 \mathrm{~d}$ and after flowing to the IR. In general, the quiverines have no known Lagrangian description, which limits the usefulness of the above framework. In the following, we study a small but rich class of examples, where we understand very well both the 4d SCFT $\mathscr{T}_{\mathbf{X}}^{4 \mathrm{~d}}$ in IIB and its $3 \mathrm{~d}$ reduction, therefore allowing us to determine the Higgs branch of $\mathcal{T}_{\mathbf{X}}^{5 \mathrm{~d}}$. Moreover, the $3 \mathrm{~d}$ theories $\mathrm{EQ}^{(4)}$ and $\mathrm{MQ}^{(5)}$, in that case, can be described by $3 \mathrm{~d}$ quiver gauge theories with (special) unitary gauge groups. We can then fruitfully compare our results to complementary approaches that use magnetic quivers derived from brane webs [41, 42, 45-52] or from the dual generalized toric polygons [30,35]. Note that our setup implicitly assumes that we obtain a well-defined $4 \mathrm{~d}$ SCFT in the IR of $D_{S^{1}} \mathcal{T}_{\mathbf{X}}^{5 \mathrm{~d}}$, at the origin of the $4 \mathrm{~d} \mathrm{CB}$; in particular, we assume the $5 \mathrm{~d}$ Higgs branch consists of a single cone. ${ }^{2}$

The relation (1.7) is realized in this setup because the deformed singularity in IIB now probes the Coulomb branch of $\mathscr{T}_{\mathbf{X}}^{4 \mathrm{~d}}$. Conversely, the crepant resolution (1.1) probes

\footnotetext{
${ }^{2}$ This need not be the case for generic canonical singularities. If the $5 \mathrm{~d}$ Higgs branch consists of several cones, they can intersect the $4 \mathrm{~d} \mathrm{CB}$ at distinct points due to instanton corrections (see e.g. [53]), but we should still be able to work locally near each point from which a quantum HB emanates. We thank the JHEP referee for pointing it out.
} 
the Higgs branch of the $4 \mathrm{~d}$ SCFT. In that context, the additional features of the resolution mentioned above $-b_{3}>0$ and residual terminal singularities - are completely understood [36]. They correspond to additional low-energy degrees of freedom (free vector multiplets and residual SCFTs, respectively) on the quantum Higgs branch of $\mathscr{T}_{\mathbf{X}}^{4 \mathrm{~d}}$. By reducing to $3 \mathrm{~d}$, this gives us some additional evidence for their $5 \mathrm{~d}$ interpretation.

\subsection{Trinion singularities and $D_{p}^{b}(\mathrm{SU}(N))$ SCFTs}

We will study in detail the following families of canonical singularities, which we dub 'trinion singularities'

$$
\mathbf{X}: \quad F\left(x, z_{1}, z_{2}, z_{3}\right)=x^{N}+x^{N-b_{1}} z_{1}^{p_{1}}+x^{N-b_{2}} z_{2}^{p_{2}}+x^{N-b_{3}} z_{3}^{p_{3}}+\cdots=0,
$$

where $p_{\alpha}(\alpha=1,2,3)$ are positive integers and $b_{\alpha} \in\{N, N-1\}$, satisfying the condition

$$
\frac{b_{1}}{p_{1}}+\frac{b_{2}}{p_{2}}+\frac{b_{3}}{p_{3}}=N
$$

The ellipsis in (1.9) stands for additional terms that are necessary in order to fully specify the isolated singularity whenever at least two of the $b_{\alpha}$ 's are equal to $N-1[37,54,55]$. In the case when $b_{1}=b_{2}=b_{3}=N$, the singularity

$$
x^{N}+z_{1}^{p_{1}}+z_{2}^{p_{2}}+z_{3}^{p_{3}}=0
$$

was studied in [56] from the IIB engineering point of view. More generally, for any allowed choice of the $b_{\alpha}$ 's, the $4 \mathrm{~d}$ SCFT $\mathscr{T}_{\mathbf{X}}^{4 \mathrm{~d}}$ associated to (1.9) takes the form

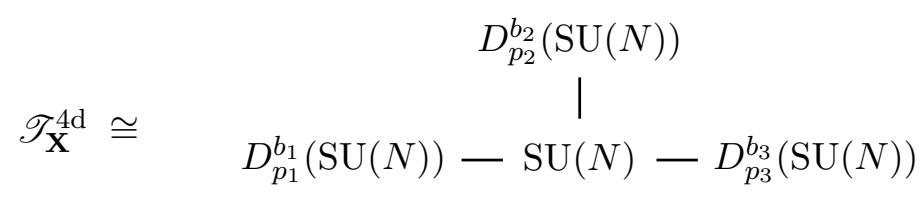

This theory consists of three $D_{p}^{b}(\mathrm{SU}(N))$ theories coupled together by gauging their common $\mathrm{SU}(N)$ global symmetry. The $4 \mathrm{~d}$ SCFTs $D_{p}^{b}(G)$, for $G$ an $A D E$ gauge group, were introduced in [57], generalizing the $D_{p}(G)$ theories studied in $[58,59]$. By construction, their flavor symmetry group is (at least) $G$. For $G=\mathrm{SU}(N)$, the $4 \mathrm{~d} \operatorname{SCFT} D_{p}^{b}(\mathrm{SU}(N))$ corresponds to a Seiberg-Witten (SW) curve $^{3}$

$$
x^{N}+x^{N-b} z^{p}=0 .
$$

One can then gauge the $\mathrm{SU}(N)$ flavor symmetries of three distinct $D_{p}^{b}(\mathrm{SU}(N))$ theories, with the condition (1.10) ensuring the vanishing of the beta function, thus preserving conformal invariance and introducing a marginal gauge coupling.

We will argue that the $3 \mathrm{~d}$ reduction of the $D_{p}^{b}(\mathrm{SU}(N))$ theory always admits a Lagrangian description as a linear quiver with unitary and special unitary gauge groups.

\footnotetext{
${ }^{3}$ More precisely, we may formulate the SW geometry in terms of a $2 \mathrm{~d} \mathcal{N}=(2,2)$ Landau-Ginzburg (LG) superpotential $W=x^{N}+x^{N-b} z^{p}$, with $(x, z) \in \mathbb{C} \times \mathbb{C}^{*}$.
} 
Upon gauging the central $\mathrm{SU}(N)$ as in (1.12), this gives us a useful description of the electric quiverine $\mathrm{EQ}^{(4)}$ as a star-shaped quiver with three legs, with $\mathrm{SU}(N)$ gauge groups at the central node. We can then obtain the magnetic quiver $\mathrm{MQ}^{(5)}$ by gauging a $\mathrm{U}(1)^{f}$ flavor symmetry. In general, one needs to carefully pick the global structure of the gauge group of the quiver $\mathrm{EQ}^{(4)}$ (and $\mathrm{MQ}^{(5)}$ ) to match the non-trivial one-form symmetries of $\mathscr{T}_{\mathbf{X}}^{4 \mathrm{~d}}[36,60]$. Correspondingly, in $5 \mathrm{~d}$, this is related to a choice of discrete 0 -form or 3 -form symmetry of $\mathcal{T}_{\mathbf{X}}^{5 \mathrm{~d}}$.

In the process of deriving the $3 \mathrm{~d}$ Lagrangians for $D_{p}^{b}(\mathrm{SU}(N))$ theories, we also introduce a new family of $4 \mathrm{~d} \mathcal{N}=2$ SCFTs with $\mathrm{SU}(N) \times \mathrm{SU}(K) \times \mathrm{U}(1)$ global symmetry which we call $\mathcal{D}_{s}^{b}(N, K)$ with $s=p / \operatorname{gcd}(p, b)$. These emerge at cusps on the conformal manifold of $D_{p}^{b}(\mathrm{SU}(N))$ theories and play a crucial role in deriving the linear quivers in $3 \mathrm{~d}$.

\subsection{Trinions, class $\mathcal{S}$ theories and generalized toric polygons}

The trinions we introduce to describe the dimensional reduction of $\mathscr{T}_{\mathbf{X}}^{4 \mathrm{~d}}$ result in Lagrangian $3 \mathrm{~d} \mathcal{N}=4$ theories which are not always 'good theories' in the Gaiotto-Witten sense [61]. The 3d quivers have $(S) \mathrm{U}(N)$ gauge nodes. In particular, for 'ugly' quivers or for 'bad' quivers (whose nodes are underbalanced), the resulting CB will not be conical, and thus will not correspond immediately to a 3d SCFT. For ugly quivers, where the underbalance is -1 , we can apply an infrared duality to rebalance the nodes, thus 'factoring out' a free sector [61]. If the quiver is bad, on the other hand, the theory needs some additional interpretation.

Given the $\mathrm{EQ}^{(4)}$ so obtained, we find the magnetic quiver $\mathrm{MQ}^{(5)}$ for the $5 \mathrm{~d}$ theory by gauging the $\mathrm{U}(1)^{f}$ symmetry. This essentially turns the $\mathrm{SU}(n)$ factors in the legs to $\mathrm{U}(n)$ factors, which can at most introduce additional ugly nodes.

For our class of trinion singularities, we can connect the properties of the magnetic quivers $\mathrm{MQ}^{(5)}$ to the features of the crepant resolution $\widetilde{\mathbf{X}}$. We make the following:

Observation 3: if $b_{3}>0$, then the resulting $\mathrm{MQ}^{(5)}$ is bad. If $b_{3}=0$ and the geometry is fully resolvable, then the quiver is good. Finally, in the case with $b_{3}=0$ and with residual terminal singularities, the quiver is ugly.

We will argue that, given a bad magnetic quivers (corresponding to a singularity with $b_{3}>0$ ), the $5 \mathrm{~d}$ physics can be extracted by considering the most singular locus on the Coulomb branch, using the CB moduli space analysis of [62]. We will also give a complementary perspective on these $3 \mathrm{~d}$ quivers by interpreting them as $3 \mathrm{~d}$ mirror duals of $4 \mathrm{~d}$ class $\mathcal{S}$ theories that are trinions (spheres with three regular punctures). The class $\mathcal{S}$ theories themselves are the $4 \mathrm{~d}$ reduction of $5 \mathrm{~d}$ SCFTs engineered from trinion-shaped $(p, q)$-brane webs, which can be efficiently described by generalized toric polygons (GTP). The specific GTPs are obtained by taking the toric diagram for $T_{N}$ and including a suitable number of white dots $[26,63]$. These class-S/GTP trinions are fully characterized by three partition of $N$ : in the GTP, the partitions encode the separation between black dots along the edges of the polygon, which equivalently encodes the distribution of 5-/7-branes in the brane-web realization. The partitions also determine the class $\mathcal{S}$ punctures and the ranks of the $\mathrm{U}\left(n_{i}\right)$ gauge nodes in the $3 \mathrm{~d}$ quiver $[64,65]$. 
The bad quivers in $3 \mathrm{~d}$ can be 'redeemed' in the following way: they have an alternative interpretation as the higgsing of a theory that is a good, by a process discussed in [66]. The 'unhiggsing' of the bad quivers will be described both in the $4 \mathrm{~d}$ class $\mathcal{S}$ setting and in the 5d GTP formalism. Starting from the unhiggsed theory, there are alternative ways to higgs back to the theory of interest, and this produces several good daughter (higgsed) theories. The badness of the original quiver reflects this fact, and in this sense a bad theory can be thought of as a collection of good theories. We give a prescription to identify the good theory in the family with the correct $3 \mathrm{~d}$ magnetic quiver whose $\mathrm{CB}$ corresponds to the HB of the $5 \mathrm{~d}$ theory $\mathcal{T}_{\mathbf{X}}^{5 \mathrm{~d}}$.

\subsection{Trinions of type $G=D, E$ and new S-dualities}

In section 7 and 8 , we discuss trinions in $4 \mathrm{~d}$ built by gauging together three $D_{p}^{b}(G)$ theories as in as in (1.12), but with the central node $G$ a special orthogonal or exceptional gauge group. We consider SCFTs for which the $G$ beta-function vanishes. In general, these theories can only be described by a Landau-Ginzburg (LG) model with five fields, which does not correspond to a threefold singularity [56,67]. If however the superpotential is such that at least one of the five fields is massive, we can integrate it out and obtain a threefold hypersurface singularity in $\mathbb{C}^{4}$, in which case the corresponding $4 \mathrm{~d}$ SCFT can be engineered by compactifying Type IIB on that singularity.

In this work, we also observe that the Type IIB geometric engineering of $4 \mathrm{~d}$ SCFTs can be used to discover new S-dualities. This can be understood as follows. From our database, we find that, in several cases, two trinions with different $G$, which a priori are distinct SCFTs, are actually described by the same hypersurface singularity (or LG model). In such a situation, the two theories are exactly equivalent - they correspond to two different-looking descriptions of the same SCFT. Since the gauge groups are different in the two descriptions, we interpret these as nontrivial examples of S-dualities, with the gauge groups in each description becoming weakly coupled at different corners of the conformal manifold as in [64, 68].

We should stress that the dualities we find are extremely nontrivial from a fieldtheoretic perspective. The most popular tool we have to understand S-dualities is the pair-of-pants decomposition of a Riemann surface in the class $\mathcal{S}$ description [64]. This however cannot be used to study the examples we will discuss in this paper since the relevant trinion SCFTs, which include special unitary quivers with exceptional shape, are not class $\mathcal{S}$ theories in general. The only other known method to probe S-dualities is the study of the degeneration limits of the Seiberg-Witten (SW) curve as in [68] (see also [6971]). This route is not viable here since the curve for quivers with exceptional shape is extremely involved [72]. Incidentally, we will also find dualities for orthosymplectic quivers with trivalent nodes, for which the curve is not known at all. Nevertheless, the Type IIB (or, equivalently LG) framework renders the S-dualities obvious. We leave a more detailed study of these $D_{p}^{b}(G)$ trinions and of their dualities for future work.

The plan of this paper is as follows: in section 2 we discuss the $4 \mathrm{~d}$ SCFTs of type $D_{p}^{b}(G)$ and their description in terms of trinion singularities as well. We discuss in detail their $3 \mathrm{~d}$ reduction. This general analysis is then applied to a large class of examples: in section 3 , we 
first discuss a class of rank 0 theories. In section 4, we study their generalization to higherrank theories which are close relatives to the higher-rank E-strings. We discuss in detail the interpretation of the terminal singularities in this class of models. In section 5 , we provide an alternative point of view on the ugly/bad quivers that arise from these trinions in $3 \mathrm{~d}$, and propose a way to redeem these theories. A full classification of $D_{p}^{b}(\mathrm{SU}(N))$ trinions is provided in section 6 . For other groups $G=D, E$, we discuss numerous examples of trinions in section 7. Finally, in section 8, we discuss non-trivial S-dualities for these $D_{p}^{b}(G)$ trinions. The appendices contain details on the geometric resolutions of canonical singularities, a proof of the $D_{p}^{b}(\mathrm{SU}(N))$-classification, as well as a list of $D_{p}^{b}(G)$-trinions for $G=D, E$.

\section{$24 \mathrm{~d} \mathcal{N}=2$ SCFTs $D_{p}^{b}(G)$, trinion singularities, and $3 \mathrm{~d}$ reduction}

The main protagonists of this section are the $4 \mathrm{~d} \mathcal{N}=2$ SCFTs $D_{p}^{b}(G)$ [57], their gauging with $\mathcal{N}=2$ vector multiplets for the $\operatorname{ADE}$ group $G$, the associated canonical singularities in IIB, and the reduction of these theories to $3 \mathrm{~d}$. It is this class of theories that will play a key role in the following sections, where we will determine the 5d SCFTs obtained from M-theory on the same singularities.

We will use the following abbreviation in writing the quivers, in $4 \mathrm{~d}$ and $3 \mathrm{~d}$, where the round nodes are gauge nodes while the square node corresponds to flavor nodes:

$\begin{array}{cccc}\bullet & \mathrm{U}(n) & \bigcirc & \mathrm{SU}(n) / \mathbb{Z}_{k} \\ \bigcirc & \mathrm{O} / \mathbb{Z}_{k} & \\ { }_{n} & \mathrm{SU}(n) & \square & \text { Flavor } \mathrm{SU}(n)\end{array}$

\subsection{Canonical singularities and 4d SCFTs}

Consider an isolated canonical singularity $\mathbf{X}$ defined by a quasi-homogeneous polynomial equation

$$
F\left(x, z_{1}, z_{2}, z_{3}\right)=0 .
$$

The family of smooth local Calabi-Yau threefolds $\{\widehat{F}=0\}$ obtained by deforming the singularity gives us the Seiberg-Witten geometry of the $4 \mathrm{~d} \mathcal{N}=2$ SCFT $\mathscr{T}_{\mathbf{X}}^{4 \mathrm{~d}}$, as engineered in Type IIB string theory (see e.g. [56, 67, 73, 74]). We have

$$
\widehat{F}=F+\sum_{l=1}^{\mu} t_{l} x^{\mathfrak{m}_{l}}=0, \quad \Omega_{3}=\frac{d x \wedge d z_{1} \wedge d z_{2} \wedge d z_{3}}{d \widehat{F}},
$$

where the deformation monomials $x^{\mathfrak{m}_{l}}$ are generators of the Milnor ring of the singularity, i.e. $\mathcal{M}=\mathbb{C}\left[x, z_{1}, z_{2}, z_{3}\right] /\langle d \hat{F}\rangle$, and $\Omega_{3}$ is the holomorphic 3-form. The condition that $\Omega_{3}$ have scaling dimension 1 fixes the scaling dimensions of the variables $x, z_{i}$, and therefore of the parameters $t_{l}$ in (2.3). In particular, the deformation parameters with scaling dimensions $\Delta\left[t_{l}\right]>1$ correspond to the Coulomb branch parameters of the $4 \mathrm{~d} \mathcal{N}=2 \mathrm{SCFT}$, while the ones with $\Delta\left[t_{l}\right]=1$ correspond to mass terms (coupling to the moment map operators in conserved-current multiplets). We denote by $\widehat{r}$ the rank of $\mathscr{T}_{\mathbf{X}}^{4 \mathrm{~d}}$ and by $f$ the 
rank of its flavor symmetry group. Note that $\mu=2 \widehat{r}+f$. Much of what is summarized here was described in more detail in [36] and will receive an in depth treatment in [37].

For the most parts of this paper, we are chiefly interested in singularities (2.2) with scaling dimensions

$$
\Delta[x]=1, \quad \Delta\left[z_{\alpha}\right]=\frac{b_{\alpha}}{p_{\alpha}}, \quad \alpha=1,2,3, \quad \Delta[F]=N,
$$

with $b_{\alpha} \in\{N, N-1\}, \forall \alpha$, and for some positive integers $p_{\alpha}$ and $N$. This spectrum is realized by the singularities (1.9), namely

$$
x^{N}+x^{N-b_{1}} z_{1}^{p_{1}}+x^{N-b_{2}} z_{2}^{p_{2}}+x^{N-b_{3}} z_{3}^{p_{3}}+\cdots=0,
$$

with the condition (1.10) being equivalent to $\Delta\left[\Omega_{3}\right]=1$. Note that the "..." terms contain necessary marginal deformations to make the singularity isolated. The Milnor ring of this singularity always includes the terms $x^{N-2}, x^{N-3}, \cdots, 1$, and the CB spectrum $\{\Delta\}$ of $\mathscr{T}_{\mathbf{X}}^{4 \mathrm{~d}}$ thus contains the dimensions

$$
\{2,3, \cdots, N\} \subset\{\Delta\} .
$$

We then have at least one marginal coupling, and the spectrum is consistent with $\mathscr{T}_{\mathbf{X}}^{4 \mathrm{~d}}$ having a weakly-coupled $\mathrm{SU}(N)$ on its conformal manifold. Indeed, we can give such a description as a marginal gauging of three $D_{p}^{b}(\mathrm{SU}(N))$ theories by this $\mathrm{SU}(N)$ [57-59]. In the following, we review these $D_{p}^{b}(\mathrm{SU}(N))$ theories and we study their circle reduction to $3 \mathrm{~d}$.

\section{2 $D_{p}^{b}(G)$ : the executive summary}

One can engineer an infinite family of $4 \mathrm{~d} \mathcal{N}=2$ SCFTs with an ADE global symmetry by compactifying Type IIB string theory on threefold hypersurface singularities in $\mathbb{C}^{3} \times \mathbb{C}^{*}$

$$
F\left(x_{1}, x_{2}, x_{3}, z\right)=0,
$$

with $\left(x_{1}, x_{2}, x_{3}\right) \in \mathbb{C}^{3}$ and $z \in \mathbb{C}^{*}$. The relevant singularities, describing an ADE singularity fibered over $\mathbb{C}^{*}$, are shown in table 1 . We will be mainly interested in the case $A_{N-1}=$ $\mathrm{SU}(N)$ in the following, but we shall also use the other cases in section 7 .

These models are indexed by the $A D E$ group $G$ (which is, in general, a subgroup of the full flavor symmetry group), a positive integer $p$, and the parameter $b$, which must take one of the values shown in table 1.

Upon deforming the singularity, similarly to (2.3), we have a smooth threefold with the holomorphic 3 -form (note that this is different from 2.3)

$$
\Omega_{3}=\frac{d x_{1} \wedge d x_{2} \wedge d x_{3} \wedge d z}{z d \hat{F}} .
$$

By construction, the deformation ring of the singularity $F=0$ contains a $G=\mathrm{ADE}$ spectrum, which is now interpreted as mass parameters for the global symmetry $G$ (in particular, they are not paired to deformation parameters of dimension $2-\Delta$, unlike the actual CB parameters). The other deformation parameters with $\Delta>1$ give us the Coulomb 


\begin{tabular}{|c|c|c|}
\hline$G$ & $F$ & $b$ \\
\hline $\mathrm{SU}(N)$ & $x_{1}^{2}+x_{2}^{2}+x_{3}^{N}+z^{p}$ & $N$ \\
\hline & $x_{1}^{2}+x_{2}^{2}+x_{3}^{N}+x_{3} z^{p}$ & $N-1$ \\
\hline $\mathrm{SO}(2 N)$ & $x_{1}^{2}+x_{2}^{N-1}+x_{2} x_{3}^{2}+z^{p}$ & $2 N-2$ \\
\hline & $x_{1}^{2}+x_{2}^{N-1}+x_{2} x_{3}^{2}+x_{3} z^{p}$ & $N$ \\
\hline
\end{tabular}

\begin{tabular}{|c|c|c|}
\hline$G$ & $F$ & $b$ \\
\hline$E_{6}$ & $x_{1}^{2}+x_{2}^{3}+x_{3}^{4}+z^{p}$ & 12 \\
\hline & $x_{1}^{2}+x_{2}^{3}+x_{3}^{4}+x_{3} z^{p}$ & 9 \\
\hline & $x_{1}^{2}+x_{2}^{3}+x_{3}^{4}+x_{2} z^{p}$ & 8 \\
\hline$E_{7}$ & $x_{1}^{2}+x_{2}^{3}+x_{2} x_{3}^{3}+z^{p}$ & 18 \\
\hline & $x_{1}^{2}+x_{2}^{3}+x_{2} x_{3}^{3}+x_{3} z^{p}$ & 14 \\
\hline$E_{8}$ & $x_{1}^{2}+x_{2}^{3}+x_{3}^{5}+z^{p}$ & 30 \\
\hline & $x_{1}^{2}+x_{2}^{3}+x_{3}^{5}+x_{3} z^{p}$ & 24 \\
\hline & $x_{1}^{2}+x_{2}^{3}+x_{3}^{5}+x_{2} z^{p}$ & 20 \\
\hline
\end{tabular}

Table 1. Hypersurface singularities $(F=0)$ realizing the $D_{p}^{b}(G)$ theories in Type IIB string theory, with all the allowed values of $b$ for each $G$.

branch spectrum of the $D_{p}^{b}(G)$ theory, and the corresponding coupling constants arise from deformations of dimensions $\Delta^{\prime}=2-\Delta<1$. In particular, a coupling constant of dimension $\Delta^{\prime}=0$ is a marginal coupling. Let us also denote by $f_{0}$ the number of parameters with $\Delta=1$, corresponding to additional conserved currents (in addition to $G$ ). Therefore, the rank of the full flavor symmetry, $G_{F}$, is given by

$$
\operatorname{rk}\left(G_{F}\right)=\operatorname{rk}(G)+f_{0} .
$$

These SCFTs are denoted by $D_{p}^{b}(G)$ [57]. The special case with $b=h^{\vee}(G)$ (the dual Coxeter number of $G)$ is often denoted by $D_{p}(G)[58,59]$. The rank $\widehat{r}$ of $D_{p}^{b}(G)$ can be deduced from

$$
\mu=2 \widehat{r}+\operatorname{rk}\left(G_{F}\right)=p \frac{\operatorname{rk}(G) h^{\vee}(G)}{b},
$$

where $\mu$ is the dimension of the ring $\mathbb{C}\left[x_{1}, x_{2}, x_{3}, z\right] /\left\langle\partial_{x_{1}} F, \partial_{x_{2}} F, \partial_{x_{3}} F, z \partial_{z} F\right\rangle$. From the spectrum of $\mathrm{CB}$ operators, we may also compute the central charges $a$ and $c$, using the relations [75]

$$
2 a-c=\frac{1}{4} \sum_{i}\left(2 \Delta_{i}-1\right),
$$

and $[57]$

$$
c=\frac{\operatorname{rk}(G)}{12 b}\left(p h^{\vee}(G)-b\right)\left(h^{\vee}(G)+1\right)-\frac{f_{0}}{12} .
$$

Another important quantity is the flavor central charge for $G$, which reads:

$$
k_{G}=2\left(h^{\vee}(G)-\frac{b}{p}\right) .
$$

Upon gauging the flavor symmetry $G$, this coefficient enters the gauge-coupling beta function. ${ }^{4}$

\footnotetext{
${ }^{4}$ Recall that, upon gauging a common $G$ symmetry of several SCFTs $\mathcal{T}_{k}$, conformal invariance requires $2 h^{\vee}(G)-\frac{1}{2} \sum_{k} k_{G}^{\mathcal{T}_{k}}=0$.
} 


\subsection{Reduction to Lagrangian theories for $G=\mathrm{SU}(N)$}

Let us now consider $D_{p}^{b}(\mathrm{SU}(N))$ on a finite-size circle, and flow to the infrared, where we expect to find a $3 \mathrm{~d} \mathcal{N}=4$ fixed point. We will argue that this $3 \mathrm{~d}$ theory can be described as the IR fixed point of a $3 \mathrm{~d} \mathcal{N}=4$ quiver gauge theory with (special) unitary gauge groups. In this sense, the $3 \mathrm{~d}$ reduction of $D_{p}^{b}(\mathrm{SU}(N))$ is a 'Lagrangian theory. ${ }^{5}$ These $3 \mathrm{~d}$ theories are also studied in [78].

Let us start by stating the main result. The linear quiver describing the $3 \mathrm{~d}$ reduction of $D_{p}^{b}(\mathrm{SU}(N))$ takes the form

$$
D_{S^{1}} D_{p}^{b}(\mathrm{SU}(N))= \begin{cases}N-\left(n_{1}\right)-\left(n_{2}\right)-\cdots-\left(n_{\ell}\right) & \text { if } b=N \text { and } \frac{p}{N} \notin \mathbb{Z}, \\ N-\left(n_{1}\right)-\left(n_{2}\right)-\cdots-\left(n_{\ell}\right)-1 & \text { otherwise. }\end{cases}
$$

The horizontal lines denote hypermultiplets in bifundamental representations, the boxes denote flavor nodes, and the gauge groups $\left(n_{k}\right)$ are either unitary or special unitary, with

$$
n_{k}=\left\lfloor N-\frac{k b}{p}\right\rfloor, \quad\left(n_{k}\right)= \begin{cases}\mathrm{U}\left(n_{k}\right) & \text { if } \frac{k b}{p} \notin \mathbb{Z}, \\ \mathrm{SU}\left(n_{k}\right) & \text { if } \frac{k b}{p} \in \mathbb{Z},\end{cases}
$$

where \lfloor\rfloor is the floor function. The length of the quiver is given by

$$
\ell= \begin{cases}p-1-\frac{p}{N} & \text { if } b=N \text { and } \frac{p}{N} \in \mathbb{Z}, \\ \left\lfloor p-\frac{p}{N}\right\rfloor & \text { if } b=N \text { and } \frac{p}{N} \notin \mathbb{Z}, \\ p-1 & \text { if } b=N-1 .\end{cases}
$$

The $\mathrm{SU}(N)$ symmetry of $D_{p}^{b}(\mathrm{SU}(N))$ is obviously realized as the flavor group on the lefthand tail of (2.14). When the quiver only contains $\mathrm{U}(n)$ factors (without the extra fundamental on the right-hand tail in (2.14), which can be viewed as an $\mathrm{SU}(1)$ factor), we have $f_{0}=0$ in $(2.9)$. The $f_{0}$ additional conserved currents in $D_{p}^{b}(\mathrm{SU}(N))$ correspond to the $\mathrm{SU}(n)$ factors in the $3 \mathrm{~d}$ quiver, including the $\mathrm{SU}(1)$ tail.

To derive this result, we will embed the $4 \mathrm{~d}$ SCFT in an asymptotically-free Lagrangian model, viewing $D_{p}^{b}(\mathrm{SU}(N))$ as an Argyres-Douglas (AD) point - i.e. as the effective lowenergy theory at a special point on the Coulomb branch of the Lagrangian theory. We then dimensionally reduce the Lagrangian theory while taking the scaling limit which brings us to the $\mathrm{AD}$ point. We first carry out this procedure for the simpler case of the $D_{2}(\mathrm{SU}(N))$ theories, arguing that the dimensional reduction gives us $3 \mathrm{~d} \mathcal{N}=4 \mathrm{SQCD}-$ that is, we have:

$$
D_{S^{1}} D_{2}(\mathrm{SU}(N))= \begin{cases}2 n-\mathrm{SU}(n) & \text { if } N=2 n, \\ 2 n-1-\mathrm{U}(n-1) & \text { if } N=2 n-1,\end{cases}
$$

where $D_{S^{1}}$ denotes the circle-reduction, which is obviously a special case of (2.14). The case $N=2 n, \mathrm{SU}(n)$ with $2 n$ flavors, is already a Lagrangian superconformal theory in $4 \mathrm{~d}$, while the case $N=2 n-1$ is a non-trivial result. We then generalize the construction

\footnotetext{
${ }^{5}$ In some instances, the $3 \mathrm{~d}$ Lagrangian can be also obtained from a $4 \mathrm{~d} \mathcal{N}=1$ Lagrangian [76, 77].
} 
to all other $D_{p}^{b}(\mathrm{SU}(N))$ models. Note that one can also check our result against other constructions, in a number of special cases. For instance, the result for $D_{2}(\mathrm{SU}(2 n-1))$ also follows from the known $3 \mathrm{~d}$ mirror dual [79]. In the case $D_{p}(\mathrm{SU}(2))$, which is the ordinary $D_{p}$ AD theory, the $3 \mathrm{~d}$ reduction similarly follow from the known $3 \mathrm{~d}$ mirror when $p$ is even [80]. We will discuss the $D_{p}(\mathrm{SU}(2))$ example in more detail momentarily.

\subsubsection{Dimensionally-reduced $D_{2}(\mathrm{SU}(N))$ theories}

The superconformal theories $D_{p}^{b}(\mathrm{SU}(N))$ can be realized as low-energy effective theories at singular points on the Coulomb branch of $4 \mathrm{~d}$ Lagrangian theories. The relevant models are linear quivers of special unitary gauge groups. The simplest non-trivial case is that of the $D_{2}(\mathrm{SU}(2 n-1))$ theories, which can be embedded in SU(n) SQCD with $N_{f}=2 n-1$ flavors. The SW curve of the Lagrangian theory can be written as

$$
y^{2}=P(x)^{2}-4 \Lambda \prod_{i=1}^{N_{f}}\left(x-m_{i}+\frac{\Lambda}{n}\right),
$$

where $P(x)=x^{n}+\sum_{i=2}^{n} u_{i} x^{n-i}$. If we set all the masses $m_{i}$ to be equal to $\frac{\Lambda}{n}-m$, and tune $P(x)$ to $P(x)=(x-(n-1) m)(x+m)^{n-1}$, the curve degenerates and the low-energy theory is $\mathrm{U}(n-1)$ with $2 n-1$ flavors. This is true for all values of $m$ except for $m=0$, where the curve degenerates further and the low-energy theory is $D_{2}(\mathrm{SU}(2 n-1))$.

What we have just described is a singular locus of the parameter space of SQCD on which the effective low energy theory is $\mathrm{U}(n-1)$ with $N_{f}=2 n-1$. Furthermore, there exists a special point on that locus, where more degrees of freedom become massless, reconstructing the spectrum of $D_{2}(\mathrm{SU}(2 n-1))$. From the perspective of the UV theory, this special point corresponds to the origin of the Coulomb branch (meaning $P(x)=x^{n}$ ) and requires setting

$$
m_{i}=\frac{\Lambda}{n}, \quad \forall i
$$

Such tuning of the flavor masses is familiar in AD theories; moreover, in general, a nontrivial quantum Higgs branch arises at the AD point [81].

Next, let us compactify the theory on a circle of radius $\widetilde{\beta}$ and reduce the theory to 3d. The discussion of the previous paragraph shows that in $4 \mathrm{~d}$ the Lagrangian theory is well approximated by $D_{2}(\mathrm{SU}(2 n-1))$ at the origin of the Coulomb branch and at energies $E \ll \Lambda$, provided that (2.19) is satisfied. We therefore want to take the 3 d limit in such a way that the $4 \mathrm{~d} R G$ scale remains finite. Since the $3 \mathrm{~d}$ and $4 \mathrm{~d}$ gauge coupling constants are related by $g_{4}^{2}=2 \pi \widetilde{\beta} g_{3}^{2}$, we should keep the following combination finite

$$
\Lambda=e^{-\frac{4 \pi}{\beta g_{3}^{2}}}
$$

in the $3 \mathrm{~d}$ limit, that is, the gauge coupling in $3 \mathrm{~d}$ should be sent to infinity as $g_{3}^{2} \sim 1 / \widetilde{\beta}$ in the limit $\widetilde{\beta} \rightarrow 0$. In particular, we should study the behaviour of the $3 \mathrm{~d} \operatorname{SU}(n)$ theory with $N_{f}=2 n-1$ in the infrared, at the origin of the Coulomb branch, but with non-zero flavor mass terms. 

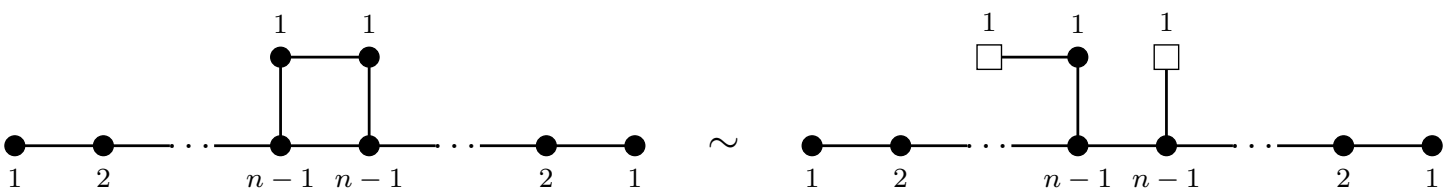

Figure 2. The $3 \mathrm{~d}$ mirror of $\mathrm{SU}(n)$ with $N_{f}=2 n-1$. All nodes are unitary and therefore we should ungauge an overall $\mathrm{U}(1)$. We choose to ungauge one of the abelian nodes above. This mirror quiver was obtained in [47] and can be derived e.g. by starting from the $3 \mathrm{~d}$ mirror of $\mathrm{SU}(n)$ with $2 n$ flavors and turning on a $\mathrm{SU}(2 n-1)$-preserving Fayet-Iliopoulos (FI) deformation.



Figure 3. The FI deformation on the red node leads to the quiver on the right-hand-side, which is the $3 \mathrm{~d}$ mirror of $\mathrm{U}(n-1)$ with $N_{f}=2 n-1$.

While the $4 \mathrm{~d}$ Coulomb branch geometry is not scale-invariant due to asymptotic freedom, the $3 \mathrm{~d}$ gauge theory flows to a $3 \mathrm{~d} \mathcal{N}=4$ superconformal fixed point, whose Coulomb branch is a hyper-Kähler cone. For the massless gauge theory, the $3 \mathrm{~d}$ Coulomb branch can be equivalently described as the Higgs branch of mirror quiver, shown in figure 2. Since we are interested in the massive theory, however, we should turn on a $\mathrm{SU}(2 n-1)$-preserving FI deformation in the mirror quiver, which gives us the quiver shown in figure 3 . This is precisely the $3 \mathrm{~d}$ mirror of $\mathrm{U}(n-1) \mathrm{SQCD}$ with $N_{f}=2 n-1$. We therefore conclude that, in contrast to the $4 \mathrm{~d}$ case, where we had a special (nonzero) value of the mass for which additional massless degrees of freedom appeared at the origin of the Coulomb branch, the low-energy theory in $3 \mathrm{~d}$ is always $\mathrm{U}(n-1) \mathrm{SQCD}$ with $N_{f}=2 n-1$, for any non-zero value of the mass. We then conclude that the 3 d reduction of the $D_{2}(\mathrm{SU}(2 n-1))$ SCFT can be described as the IR fixed point of $3 \mathrm{~d} \mathcal{N}=4 \mathrm{U}(n-1)$ SQCD with $N_{f}=2 n-1$ flavors.

\subsection{2 $D_{p}^{b}(\mathrm{SU}(N))$ theories and $3 \mathrm{~d}$ quivers}

In order to discuss the general case, let us first discuss the structure of the deformation ring of the Type IIB geometry that engineers the $4 \mathrm{~d}$ SCFT. We have ${ }^{6}$

$$
D_{p}^{b}(\mathrm{SU}(N)): \quad \begin{cases}x^{N}+z^{p}+u_{k l} z^{k} x^{l}=0 & \text { if } b=N, \\ x^{N}+x z^{p}+u_{k l} z^{k} x^{l}=0 & \text { if } b=N-1 .\end{cases}
$$

The deformation coefficients have scaling dimensions

$$
\Delta\left[u_{k l}\right]=N-\frac{k b}{p}-l .
$$

\footnotetext{
${ }^{6}$ For the case of $\mathrm{SU}(N)$, we ignore the terms $x_{1}$ and $x_{2}$ in $F\left(x_{1}, x_{2}, x_{3}, z\right)$ since they do not appear in deformation terms, and we take $x \equiv x_{3}$.
} 
We can check that $k$ runs from 1 to $\ell$ or $\ell+1$, corresponding to the two cases in (2.14), respectively, with $\ell$ defined in (2.16). In the second case, the monomial $z^{\ell+1}$ has dimension $\ell$, and corresponds to the mass term associated to the fundamental flavor on the right-hand tail of the $3 \mathrm{~d}$ quiver (2.14).

Let us collect all the terms with the same power of $z$. As we already mentioned, the $k=0$ deformations terms are associated with the $\mathrm{SU}(N)$ flavor current. On the other hand, for each $k>1$, the monomials

$$
z^{k} x^{l}, \quad l=0, \cdots, n_{k}-1
$$

have dimension $\Delta \geq 1$, for $n_{k}$ as defined in (2.15). If, furthermore, $\frac{k b}{p} \in \mathbb{Z}$, the coefficients $u_{k l}$ have the integer-valued dimensions $\Delta\left[u_{k l}\right]=n_{k}, n_{k}-1, \cdots, 2,1$. The last coefficient is then again a mass term, while the other ones are the CB parameters for a conformallygauged $\mathrm{SU}\left(n_{k}\right)$ factor of the $4 \mathrm{~d} \mathrm{SCFT}$. On the other hand, if $\frac{k b}{p} \notin \mathbb{Z}$, we have the non-integer dimensions $\Delta=N-\frac{k b}{p}-l$. We claim that these $n_{k}$ CB operators will correspond to a $\mathrm{U}\left(n_{k}\right)$ factor in the $3 \mathrm{~d}$ quiver description. We have already shown this in the case $p=2$. In the following, we argue for our $3 \mathrm{~d}$ quiver description in the general case.

In order to do this, it is convenient to 'break down' the $D_{p}^{b}(\mathrm{SU}(N))$ theory into smaller building blocks. Indeed, whenever $p$ and $b$ are not mutually prime, there exists a 'partially Lagrangian' description of $D_{p}^{b}(N)$ that includes the $\mathrm{SU}\left(n_{k}\right)$ gauge groups for $k$ such that $\frac{k b}{p} \in \mathbb{Z}$. Indeed, consider

$$
m \equiv \operatorname{gcd}(p, b)>1, \quad s \equiv \frac{p}{m} .
$$

We then have the first $\mathrm{SU}\left(n_{k}\right) \equiv \mathrm{SU}(K)$ node at $k=s$, and the SCFT can be broken down as

$$
D_{p}^{b}(\mathrm{SU}(N)) \cong \mathcal{D}_{s}^{b}(N, K)-\mathrm{SU}(K)-D_{\widehat{p}}^{b}(\mathrm{SU}(K))
$$

with

$$
K=N-\frac{b}{m}, \quad \widehat{p}=p-s .
$$

The theory $\mathcal{D}_{s}(N, K)$ is an 'isolated' SCFT without a conformal manifold, and with an $\mathrm{SU}(N) \times \mathrm{SU}(K) \times \mathrm{U}(1)$ flavor symmetry. Its $\mathrm{CB}$ spectrum is entirely non-integer. It is formally given as a 'quotient' of two $D_{p}^{b}(\mathrm{SU}(N))$ theories

$$
\mathcal{D}_{s}^{b}(N, K) \cong \frac{D_{p}^{b}(\mathrm{SU}(N))}{D_{\widehat{p}}^{b}(\mathrm{SU}(K))},
$$

where, in (2.25), we indicated that, to recover $D_{p}^{b}(\mathrm{SU}(N))$, we must glue $\mathcal{D}_{s}^{b}(N, K)$ to $D_{\hat{p}}^{b}(\mathrm{SU}(K))$ by gauging the common $\mathrm{SU}(K)$ flavor symmetry. To derive our $3 \mathrm{~d}$ quiver description, we then need to derive the $3 \mathrm{~d}$ quiver for $\mathcal{D}_{s}^{b}(N, K)$, which takes the form

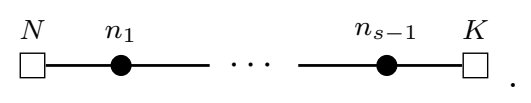

Finally, we will just need to consider the case $D_{p}^{b}(N)$ with $\operatorname{gcd}(p, b)=1$. 
Let us now assume $b=N$, for simplicity. (The case $b=N-1$ is similar.) Formally, we can view the theory $\mathcal{D}_{s}(N, K)$ as engineered from the non-isolated singularity

$$
x^{N}+x^{K} z^{s} .
$$

We then have $\mathcal{D}_{s}(N, 0)=D_{s}^{N}(\mathrm{SU}(N))$ and $\mathcal{D}_{s}(N, 1)=D_{s}^{N-1}(\mathrm{SU}(N))$ as special cases. We would like to realize the SCFTs $\mathcal{D}_{s}(N, K)$ at special points on the $\mathrm{CB}$ of a Lagrangian theory.

Let us start with the case $K=N-1$, which is simpler to analyze. This model occurs as in $(2.25)$ for $D_{p}(\mathrm{SU}(N))$ with $p=s N$, for $s$ integer. Then, the 4 d Lagrangian theory of interest is the linear quiver

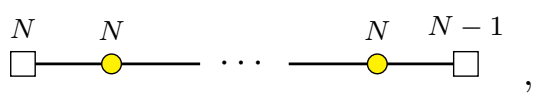

where the number of $\mathrm{SU}(N)$ gauge groups is $s-1$. Notice that all the $\mathrm{SU}(N)$ gauge groups have a vanishing beta function except for the rightmost one, which has $N_{f}=2 N_{c}-1$ and is asymptotically free. We can therefore apply the results of section 2.3 .1 and argue that, when we go down to $3 \mathrm{~d}$, the gauge group is effectively replaced by $\mathrm{U}(N-1)$. At this stage, the second rightmost node is no longer conformal, and we can reiterate the procedure. We therefore end up in $3 \mathrm{~d}$ with the linear quiver

$$
D_{S^{1}} \mathcal{D}_{p}(N, N-1)=\square \stackrel{N N-1}{\square} \cdots \stackrel{N-1 N-1}{\bullet} \square .
$$

This theory is good in the Gaiotto-Witten (GW) sense, and it therefore has a conventional IR fixed point at the origin of its Coulomb branch, with a Higgs-branch global symmetry $\mathrm{SU}(N) \times \mathrm{SU}(N-1) \times \mathrm{U}(1)$, as expected. Note the enhancement to $\mathrm{SU}(2 N-1)$ for $p=2$; in that case, the theory is actually equivalent to $D_{2}(\mathrm{SU}(2 N-1))$, and we recover the result (2.17). Let us also note that, in this special case with $K=N-1$, we can provide an alternative definition of the theory using class- $\mathcal{S}$ technology, which confirms our result (2.31) for the 3 d quiver. ${ }^{7}$

For $K<N-1$, we can find a similar Lagrangian UV completion from which we can argue for the $3 \mathrm{~d}$ quiver description. The $4 \mathrm{~d}$ quiver is of the form

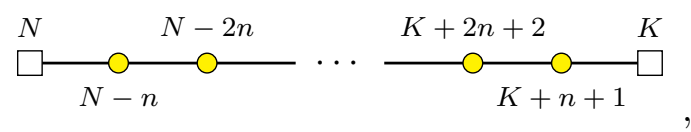

where $n=\left\lfloor\frac{N-K}{s}\right\rfloor$. On the one hand, starting from the left with $N$ fundamentals of $\mathrm{SU}(N-n)$, we decrease the number of colors by $n$ at each step. On the other hand, starting from the right-hand tail of the quiver with the $K$ fundamentals of $\mathrm{SU}(K+n+1)$,

\footnotetext{
${ }^{7}$ Consider the $\mathcal{N}=(2,0)$ theory of type $A_{N-1}$ on a sphere with a regular full puncture and an irregular puncture of type III labelled by $s+1$ partitions of $N$. In the notation of [80], the partitions are $Y_{s+1}=$ $\cdots=Y_{2}=(N-1,1)$ and $Y_{1}=\left(1^{N}\right)$. It is easy to check that the CB spectra of this class- $\mathcal{S}$ theory and of the $\mathcal{D}_{s}(N, N-1)$ model are identical. We then find that the $3 \mathrm{~d}$ mirror constructed following the algorithm of [80] is precisely the mirror dual of the quiver (2.31).
} 
we increase the number of colors by $n+1$ as we move to the left. These two tails meet at the center at the $(N-K-n s)$-th gauge group from the right, and all the gauge groups are conformal except for the 'central' one. One can check that the SW curve for this Lagrangian theory includes all possible deformation terms for the $\mathcal{D}_{s}(N, K)$ theory. Locally, the nonconformal 'central' node is $\mathrm{SU}\left(N_{c}\right)$ with $N_{f}=2 N_{c}-1$ flavors, and we can therefore replace that gauge group with an $\mathrm{U}\left(N_{c}-1\right)$ node when we dimensionally reduce to $3 \mathrm{~d}$. We can then again sequentially replace all $S U$ groups with unitary groups of the same rank.

Once all the gauge groups in the quiver (2.32) have been replaced by unitary groups, some nodes might become ugly in the sense of Gaiotto-Witten [61], ${ }^{8}$ in which case we can apply the local duality

$$
\mathrm{U}\left(N_{c}\right), N_{f}=2 N_{c}-1 \quad \longleftrightarrow \quad \mathrm{U}\left(N_{c}-1\right), N_{f}=2 N_{c}-1 \oplus \mathbb{H},
$$

where $\mathbb{H}$ indicates one twisted hypermultiplet. This procedure generates a collection of twisted hypermultiplets besides the interacting sector. The interacting sector turns out to be described by the unitary quiver

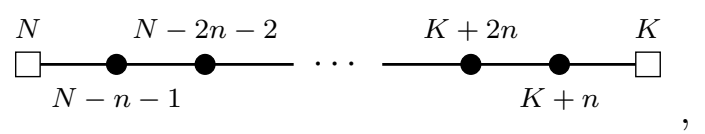

where again $n=\left\lfloor\frac{N-K}{s}\right\rfloor$ and all nodes are clearly good. We find that (2.34) is equivalent to the interacting sector of the following quiver

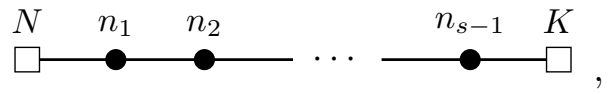

where $n_{1}=N-\left\lceil\frac{N-K}{s}\right\rceil, n_{2}=N-\left\lceil 2 \frac{N-K}{s}\right\rceil$, and so on. Notice that, although the interacting sectors in the IR are the same, the number of twisted hypermultiplets we find starting from (2.32) and reducing to $3 \mathrm{~d}$ does not necessarily match the number of twisted hypermultiplets we get from (2.35) by applying the duality (2.33). The reason is that, in general, the rank of the Lagrangian theory (2.32) is larger than that of the corresponding $\mathcal{D}_{s}(N, K)$ model. Therefore, from (2.32), we in general obtain additional twisted hypermultiplets. This is due to the fact that, besides the $\mathcal{D}_{s}(N, K)$ sector, the lowenergy theory on the $4 \mathrm{~d} \mathrm{CB}$ of (2.32) includes some abelian vector multiplets, which become twisted hypermultiplets upon dimensional reduction. We therefore claim that (2.35) is the correct dimensional reduction of $\mathcal{D}_{s}(N, K)$, since the ranks are the same. The number of colors in $(2.35), n_{k}=N-\left\lceil\frac{(N-K) k}{s}\right\rceil$, are precisely the ones specified by $(2.15)$ in our general algorithm.

Next, let us discuss the dimensional reduction of $D_{p}(\mathrm{SU}(N))$ with $N$ and $p$ coprime, which does not have any conformal manifold. For $p<N$, the analog of (2.32) is a quiver with $p-1$ gauge groups of the form:

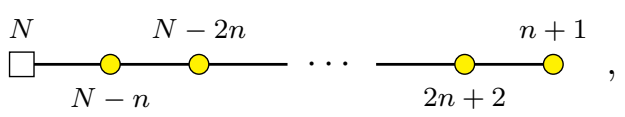

\footnotetext{
${ }^{8}$ Recall that by defining $e \equiv N_{f}-2 N_{c}$ the balance of a node, then quivers with all nodes with $e=0$ are good, if there is $e=-1$ they are ugly and $e<-1$ bad.
} 
where $n=\left\lfloor\frac{N}{p}\right\rfloor$. This can be viewed as the $K=0$ limit of (2.32). There is again only one asymptotically free node, and by the same argument as above we obtain the linear quiver with unitary nodes:

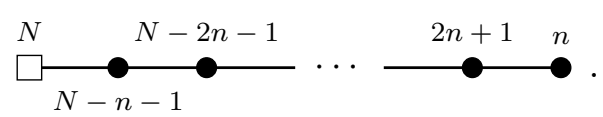

For $p>N$, we take the following $4 \mathrm{~d}$ linear quiver:

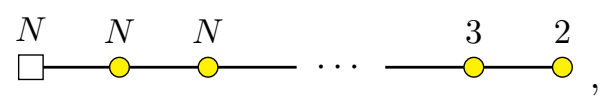

containing $\ell=\left\lfloor p-\frac{p}{N}\right\rfloor$ gauge groups. Here, the only new ingredient is the fact that, while turning $\mathrm{SU}(n)$ groups into $\mathrm{U}(n-1)$ (starting from the last $\mathrm{SU}(N)$ factor), we have to deal with an $\mathrm{SU}(2)$ gauge group with 2 flavors, at the right-hand tail of the quiver, which is not of the form $\mathrm{SU}\left(N_{c}\right)$ with $2 N_{c}-1$ flavors. In this case, to flow to the $D_{p}(\mathrm{SU}(N))$ theory, we should move onto the Coulomb branch of $\mathrm{SU}(2)$ with 2 flavors to its $\mathrm{AD}$ point, where the effective theory is $\left(A_{1}, A_{3}\right)$. Upon dimensional reduction to $3 \mathrm{~d}$, the $\mathrm{SU}(2)$ group is then replaced by SQED with 2 flavors; in conclusion, the naive substitution $\mathrm{SU}\left(n_{k}\right)$ to $\mathrm{U}\left(n_{k}-1\right)$ throughout the quiver, from $4 \mathrm{~d}$ to $3 \mathrm{~d}$, is the correct one. The limiting case is $N=2$, the $D_{p}(\mathrm{SU}(2))$ theory, which we discuss separately below, as an example.

By successive application of the decomposition (2.25), one can derive the $3 \mathrm{~d}$ quiver description for arbitrary $N$ and $p$. This then completes our derivation of the $3 \mathrm{~d}$ 'electric quiver' (2.14) for the $4 \mathrm{~d} \operatorname{SCFT} D_{p}(\mathrm{SU}(N))$. The derivation of the 3 d quiver $D_{p}^{b}(\mathrm{SU}(N))$ for $b=N-1$ is similar; in particular, for $m=\operatorname{gcd}(p, N-1)=1$, the simply have the model $\mathcal{D}_{p}(N, K)$ discussed above with $K=1$.

\subsubsection{Examples}

Let us illustrate the general discussion from the last section by providing examples for reductions that lead to $3 \mathrm{~d}$ linear quivers.

Example 1: $\boldsymbol{N}=\mathbf{2}, \boldsymbol{D}_{p}$ AD theory. As our first example, consider the case $D_{p}(\mathrm{SU}(2))$, which is the ordinary $\left(A_{1}, D_{p}\right)$ Argyres-Douglas theory. For $p=2 n$, we have $D_{2 n}(\mathrm{SU}(2))=$ $\mathcal{D}_{2 n}(2,1)$, and the $3 \mathrm{~d}$ quiver is given by $(2.31)$ with $N=2$, namely

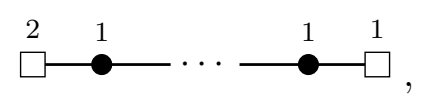

with $n-1 \mathrm{U}(1)$ factors. This agrees with the known result; see e.g. [80, 82, 83] and also [84] for a recent discussion.

For $p=2 n+1$, instead, we embed the AD theory within an 4 d linear quiver with $\mathrm{SU}(2)^{n}$ gauge group, which is the $N=2$ specialization of (2.38). The 3 d quiver then takes the form

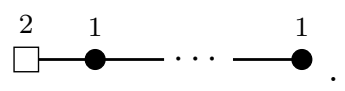

In this case, the rightmost gauge node is 'ugly'. Upon repeated use of the GW duality (2.33), we are left with SQED U(1), $N_{f}=2$ plus $n-1$ free twisted hypermultiplets. This agrees with the $3 \mathrm{~d}$ reduction of $D_{2 n+1}$ first derived in [84]. 
Example 2: the $\boldsymbol{D}_{\mathbf{7}}(\mathrm{SU}(5))$ model. For $N=5, p=7$, the $4 \mathrm{~d}$ Lagrangian theory is given by (2.38), with $\ell=5$. The 3 d reduction then gives

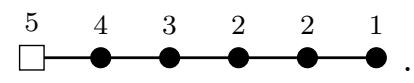

This quiver is ugly; upon application of the GW duality, we obtain the good quiver

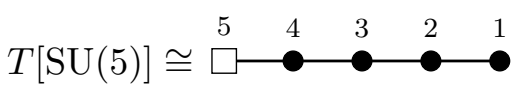

plus two twisted hypermultiplets.

Example 3: the $\boldsymbol{D}_{\mathbf{6}}(\mathbf{S U}(\mathbf{9}))$ model. In this case, our general algorithm (2.14) gives the $3 \mathrm{~d}$ quiver



Note that the $4 \mathrm{~d}$ theory is decomposed as:

$$
\mathcal{D}_{2}(9,6)-\mathrm{SU}(6)-\mathcal{D}_{2}(6,3)-\mathrm{SU}(3)-D_{2}(\mathrm{SU}(3)),
$$

in terms of the 'prime factors' $\mathcal{D}_{p}(N, K)$.

Example 4: the $D_{14}(\mathrm{SU}(8))$ model. This example is a bit more elaborate. We have $N=8, p=14$, so that $m=\operatorname{gcd}(14,8)=2$ and $s=\frac{p}{m}=7$; we then have a one-dimensional conformal manifold. According to (2.14), the $3 \mathrm{~d}$ quiver reads



This quiver is ugly, and it is equivalent to the good quiver

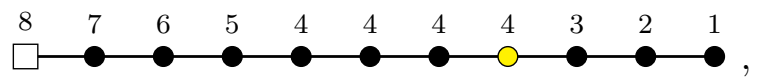

plus 6 twisted hypermultiplets. The 4d theory decomposes as

$$
D_{14}(\mathrm{SU}(8)) \cong \mathcal{D}_{7}(8,4)-\mathrm{SU}(4)-D_{7}(\mathrm{SU}(4))
$$

The $D_{7}(\mathrm{SU}(4))$ tail has the equivalent $3 \mathrm{~d}$ description as

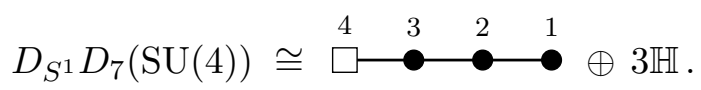

The $\mathcal{D}_{7}(8,4)$ sector has the $3 \mathrm{~d}$ description

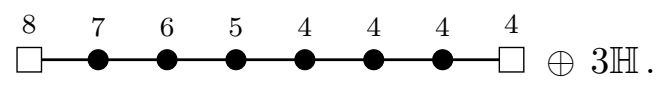

This gives us a non-trivial example of the discussion below (2.35). According to (2.32), the $\mathcal{D}_{7}(8,4)$ SCFT can be embedded into the 4 d Lagrangian theory

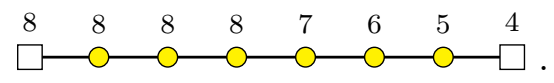


Upon going to $3 \mathrm{~d}$, we then find the quiver:

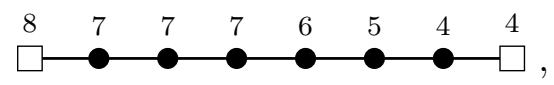

whose rank is equal to the rank of $\mathcal{D}_{7}(8,4)$ plus 3 . But the quiver $(2.51)$ is ugly and equivalent to the good quiver shown in (2.49) plus six twisted hypermultiplets. Therefore, the $3 \mathrm{~d}$ infrared description that we obtain starting from $(2.50)$ is equivalent to the theory (2.49) plus three twisted hypermultiplets. This additional free sector accounts for the rank mismatch between the $4 \mathrm{~d}$ Lagrangian theory $(2.50)$ and the $\mathcal{D}_{7}(8,4)$ model.

\subsubsection{Higgs branch and anomaly matching}

The Higgs branch of $D_{p}^{b}(\mathrm{SU}(N))$ can be directly constructed from the 3d quiver (2.14), which means that the quantum Higgs branch of the 4d SCFT can be obtained as a hyperkähler quotient. The Higgs branch quaternionic dimension is then given by

$$
\widehat{d}_{\mathrm{H}}\left(D_{p}^{b}(\mathrm{SU}(N))\right)=\sum_{k=1}^{\ell+1}\left(n_{k} n_{k-1}-n_{k}^{2}\right)+f_{0}
$$

where we have $n_{0} \equiv N$ and $n_{k+1}=0$ if $b=N$ and $\frac{p}{N} \in \mathbb{Z}$ and $n_{k+1}=1$ otherwise. This integer should be compared to the quantity

$$
24(c-a)=\frac{(N-1)(2 N+5)(N p-b)}{2 b}-\frac{5}{2} f_{0}-6 \sum_{i=1}^{\widehat{r}} \Delta_{i},
$$

which is not always an integer. Whenever the low-energy theory on the 4d Higgs branch consists of only $\widehat{d}_{\mathrm{H}}$ hypermultiplets, we must have

$$
24(c-a)=\widehat{d}_{\mathrm{H}}\left(D_{p}^{b}(\mathrm{SU}(N))\right),
$$

by anomaly matching on the HB. If this equality fails, this means that the Higgs branch theory is non-trivial: it must contain free vector multiplets and/or a 4d SCFT without a Higgs branch.

The equality (2.54) is highly non-trivial, but it can be checked in many example. Moreover, we find that $24(c-a) \neq \widehat{d}_{\mathrm{H}}$ whenever the $3 \mathrm{~d}$ quiver is ugly in the GW sense that is, the IR fixed point contains a free sector of twisted hypermultiplets. This is perhaps expected from the point of view of the $4 \mathrm{~d}$ Higgs-branch theory, since free vector multiplets will reduce to a free sector and, less trivially, any $4 \mathrm{~d} \mathcal{N}=2$ SCFT with an empty Higgs branch is expected to flows to twisted hypermultiplets as well. ${ }^{9}$

On the other hand, the $3 \mathrm{~d}$ quiver is never bad (in the Gaiotto-Witten sense). This can be seen as follows. The $j$-th gauge group of the quiver is $\mathrm{U}\left(n_{j}\right)$ where $n_{j}=\left\lfloor N-j \frac{N}{p}\right\rfloor$.

\footnotetext{
${ }^{9}$ This is assuming that non-trivial $3 \mathrm{~d} \mathcal{N}=4$ fixed points with either an empty HB or an empty CB do not exist. (Here, a 'trivial theory' is a theory of free (twisted) hypermultiplets or a discrete gauging thereof.)
} 
The number of flavors is given by the rank of the $(j-1)$-th and $(j+1)$-th gauge groups, which are respectively $\left\lfloor N-(j-1) \frac{N}{p}\right\rfloor$ and $\left\lfloor N-(j+1) \frac{N}{p}\right\rfloor$. Using the inequalities

$$
\left\lfloor N-(j-1) \frac{N}{p}\right\rfloor \geq n_{j}+\left\lfloor\frac{N}{p}\right\rfloor, \quad\left\lfloor N-(j+1) \frac{N}{p}\right\rfloor \geq n_{j}-1-\left\lfloor\frac{N}{p}\right\rfloor,
$$

it is easy to see that every node in the quiver is either good or ugly.

\subsection{Quiverines from marginally-gauged $D_{p}^{b}(\mathrm{SU}(N))$-trinions}

\subsubsection{Trinion singularities}

The 'trinion singularity' (1.9) engineers the $\mathrm{SU}(N)$ gauging of three $D_{p}^{b}(\mathrm{SU}(N))$ theories, as displayed in (1.12). The condition for the vanishing of the $\mathrm{SU}(N)$ beta-function is given by $(1.10)$

$$
\begin{aligned}
\beta_{\mathrm{SU}(N)} & =2 N-\frac{1}{2} \sum_{\alpha=1}^{3} k_{\mathrm{SU}(N)}\left(D_{p_{\alpha}}^{b_{\alpha}}(\mathrm{SU}(N))\right) \\
& =-N+\sum_{\alpha=1}^{3} \frac{b_{\alpha}}{p_{\alpha}}=0 .
\end{aligned}
$$

The trinion singularity takes the following form, depending on whether we have zero, one, two or three distinct $D_{p}^{b}(\mathrm{SU}(N))$ factors with $b=N-1$, respectively:

$$
\begin{array}{ll}
\mathrm{I}\left(p_{1}, p_{2}, p_{3}, N\right) & : F=x^{N}+z_{1}^{p_{1}}+z_{2}^{p_{2}}+z_{3}^{p_{3}} \\
\mathrm{II}\left(p_{1}, p_{2}, N, p_{3}\right) & : F=x^{N}+z_{1}^{p_{1}}+z_{2}^{p_{2}}+x z_{3}^{p_{3}} \\
\operatorname{VIII}\left(p_{1}, N, p_{2}, p_{3}\right) & : F=x^{N}+z_{1}^{p_{1}}+x z_{2}^{p_{2}}+x z_{3}^{p_{3}}+\cdots \\
\operatorname{XIV}\left(N, p_{1}, p_{2}, p_{3}\right) & : F=x^{N}+x z_{1}^{p_{1}}+x z_{2}^{p_{2}}+x z_{3}^{p_{3}}+\cdots .
\end{array}
$$

The notation in terms of 'types' I, II, VIII and XIV follows the Yau-Yu classification [54, 85]; see [37] for a detailed discussion.

For the type I singularities, the only solutions to the condition:

$$
\frac{1}{p_{1}}+\frac{1}{p_{2}}+\frac{1}{p_{3}}=1
$$

are well-known to be $\left(p_{\alpha}\right)=(3,3,3),(2,4,4)$ and $(2,3,6)$. This gives three infinite families of trinions, indexed by $N$. These models will be denoted by

$$
\begin{array}{ll}
E_{6}^{(k)}: & x_{1}^{3}+x_{2}^{3}+x_{3}^{3}+x_{4}^{3+k}, \\
E_{7}^{(k)}: & x_{1}^{2}+x_{2}^{4}+x_{3}^{4}+x_{4}^{4+k}, \\
E_{8}^{(k)}: & x_{1}^{2}+x_{2}^{3}+x_{3}^{6}+x_{4}^{6+k},
\end{array}
$$

with $N=3+k, 4+k$ and $6+k$, respectively, and for any $N>1$. The corresponding $4 \mathrm{~d}$ SCFTs $\mathcal{T}_{\mathbf{X}}^{5 \mathrm{~d}}$ were called $\left(E_{n}^{(1,1)}, \mathrm{SU}(N)\right)$ in [56]. The solutions to (2.56) for the trinions of type II, VIII and XIV will be classified in section 6 below.

The singularities (2.57), with holomorphic 3-form given by (2.3), are such that $\Delta[x]=1$ and $\Delta\left[z_{\alpha}\right]=\frac{b_{\alpha}}{p_{\alpha}}$. Each $D_{p}^{b}(\mathrm{SU}(N))$ matter sector is described by the terms $x^{N}+x^{N-b} z^{p}$, 
and the CB spectrum of $\mathcal{T}_{\mathbf{X}}^{5 \mathrm{~d}}$ is precisely as expected - it is the direct sum of the $\mathrm{CB}$ spectrum of the three $D_{p}^{b}(\mathrm{SU}(N))$ factors, plus the spectrum $\{2, \cdots, N\}$ from the $\mathrm{SU}(N)$ vector multiplet. Note that we could describe marginally-gauged theories involving other $D_{p}^{b}(G)$ factors similarly, as we will discuss in section 7 .

\subsubsection{The $3 \mathrm{~d}$ reduction of $\mathscr{T}_{X}^{4 \mathrm{~d}}$}

The trinion singularities (2.57) engineers the SCFTs $\mathcal{T}_{\mathbf{X}}^{5 \mathrm{~d}}$ in IIB with an $\mathrm{SU}(N)$ gaugetheory description:

$$
\begin{gathered}
D_{p_{2}}^{b_{2}}(\mathrm{SU}(N)) \\
\mathscr{T}_{\mathbf{X}}^{4 \mathrm{~d}} \cong D_{p_{1}}^{b_{1}}(\mathrm{SU}(N))-\mathrm{SU}(N)-D_{p_{3}}^{b_{3}}(\mathrm{SU}(N))
\end{gathered}
$$

Since we have just given an explicit description of the $3 \mathrm{~d}$ reduction of $D_{p}^{b}(\mathrm{SU}(N))$ as a $3 \mathrm{~d} \mathcal{N}=4$ linear (special) unitary quiver, it is tempting to simply gauge together three such linear quivers along their common $\mathrm{SU}(N)$ flavor node, thus obtaining a star-shaped $3 \mathrm{~d}$ quiver, which should give us an explicit description of the electric quiverine $\mathrm{EQ}^{(4)}$ for $\mathscr{T}_{\mathbf{X}}^{4 \mathrm{~d}}$ on a circle. This is indeed the prescription we will follow in this work. There are a number of important caveats, however, which we will address in the following.

If the $4 \mathrm{~d}$ quiver is already a Lagrangian SCFT, then there is no issue with reducing the Lagrangian to $3 \mathrm{~d}$, and we obtain a star-shaped quiver with $\mathrm{SU}(n)$ gauge groups. The only fully Lagrangian $D_{p}^{b}(\mathrm{SU}(N))$ theories are [59]

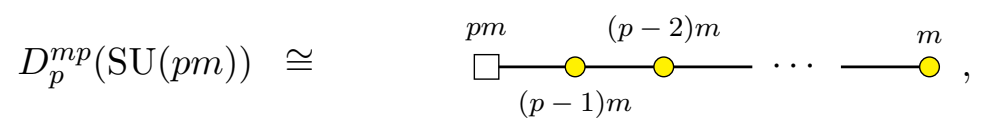

and $[57]$

$$
D_{p}^{m p}(\mathrm{SU}(p m+1)) \cong \quad \underset{(p-1) m+1}{\square m+1} \cdots \stackrel{m+1}{\square} \stackrel{1}{\square} .
$$

A well-known class of examples consists of the 4d SCFT associated with the singularities $I(3,3,3,3 m), I(2,4,4,4 m)$ and $I(2,3,6,6 m)$ in $(2.57)$, which are $\widehat{E}$-shaped 4 d superconformal quivers with $\prod_{i} \mathrm{SU}\left(d_{i} m\right)$ gauge group, with $d_{i}$ the Dynkin labels [86]:

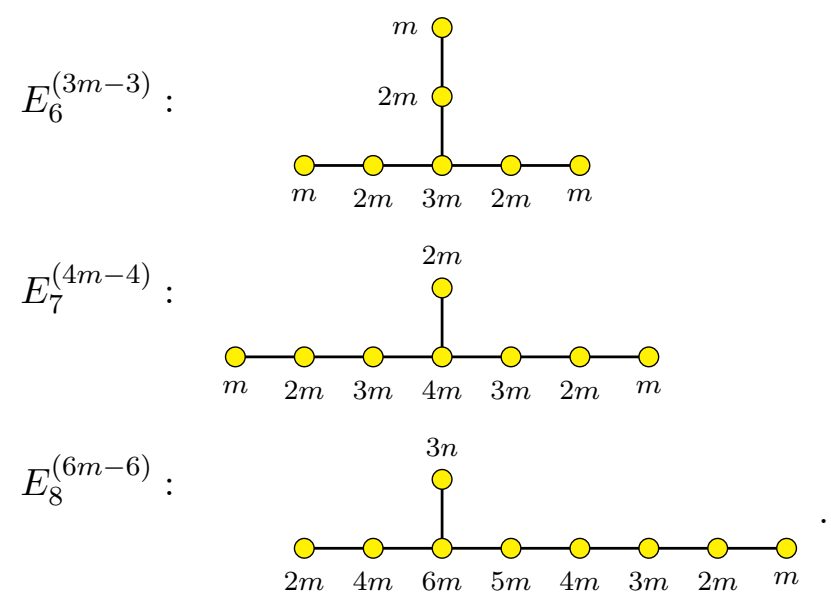


All the legs are of the form (2.61). E.g. the $\widehat{E}_{6}$-shaped quiver has three legs corresponding to $D_{3}(\mathrm{SU}(3 n))$.

In general, however, the $D_{p}^{b}(\mathrm{SU}(N))$ legs in (2.60) are not Lagrangian SCFTs, and we are not guaranteed that the $3 \mathrm{~d}$ star-shaped quiver with unitary groups gives the most useful description of the electric quiverine $\mathrm{EQ}^{(4)}$. As we will see in examples, sometimes the $3 \mathrm{~d}$ $\mathcal{N}=4$ quiver so-obtained is 'bad' in the GW sense, and therefore in need of some additional interpretation. This is expected to occur when the Higgs branch of the $4 \mathrm{~d} \mathcal{N}=2$ SCFT $\mathscr{T}_{\mathbf{X}}^{4 \mathrm{~d}}$ contains massless degrees of freedom besides the hypermultiplets. Geometrically, this corresponds to having a crepant resolution $\widetilde{\mathbf{X}}$ with residual terminal singularities and/or with 3-cycles, as we discussed in the introduction. In those instances, we will show in the following sections that the interpretation of the magnetic quiver $\mathrm{MQ}^{(5)}$ requires further refinement - e.g. we will need to 'redeem' the bad quivers, by 'unhiggsing' them first and then applying an alternative higgsing as in [66]. Let us also note that, in all the examples below, the 'badness' of the magnetic quiver for $\mathcal{T}_{\mathbf{X}}^{5 \mathrm{~d}}$ is related to the 3 -cycles rather than to the terminal singularities.

\section{Terminal singularities and rank 0 5d SCFTs}

We now turn to 5d SCFTs obtained from M-theory on canonical singularities. The first class of models we study correspond to $5 \mathrm{~d}$ rank-zero SCFTs $\mathcal{T}_{\mathbf{X}}^{5 \mathrm{~d}}$ engineered by terminal singularities in M-theory. We will discuss the singularities shown in table 2, following the logic reviewed in the introduction (see figure 1). These terminal singularities, denoted by $E_{n}^{(k)}$ with $k<0$, will appear in later sections as residual singularities on the crepant resolution of other singularities, including the $E_{n}^{(k)}$ models with $k>0$. For specific values of $k$ these were discussed in (2.63).

Using the results of the previous section, we directly find that the $4 \mathrm{~d}$ theories $\mathscr{T}_{\mathbf{X}}^{4 \mathrm{~d}}$ in Type IIB can be described as a conformal gauging of three $D_{p}(\mathrm{SU}(n)) 4 \mathrm{~d}$ SCFTs. We then give the electric quiver $\mathrm{EQ}^{(4)}$ and the magnetic quiver $\mathrm{MQ}^{(5)}$ as star-shaped quivers. By analysing the Coulomb branch of $\mathrm{MQ}^{(5)}$, we find that the Higgs branch of $\mathcal{T}_{\mathbf{X}}^{5 \mathrm{~d}}$ takes the form

$$
\mathrm{HB}\left[\mathcal{T}_{\mathbf{X}}^{5 \mathrm{~d}}\right] \cong \mathbb{H}^{d_{\mathrm{H}}} / \mathbb{Z}_{k}
$$

The integer $k$ (which could be $k=1$ ) and the action of $\mathbb{Z}_{k}$ is determined by the spectrum of line operators in $\mathscr{T}_{\mathbf{X}}^{4 \mathrm{~d}}$, as we will see. Therefore, the electric quiverine of $\mathcal{T}_{\mathbf{X}}^{5 \mathrm{~d}}, \mathrm{EQ}^{(5)}$, consists of (in general) discretely gauged $3 \mathrm{~d} \mathcal{N}=4$ hypermultiplets. We then conclude that the $5 \mathrm{~d}$ theory $\mathcal{T}_{\mathbf{X}}^{5 \mathrm{~d}}$ also consists of a discrete gauge theory of hypermultiplets, modulo any 'hidden' 5d sector without any moduli space, which, if it exists, cannot be captured by our analysis.

\section{$3.1 \quad \mathscr{T}_{X}^{4 \mathrm{~d}}$ and trinion-SCFTs for rank $0 E_{n}^{(k)}$ theories}

The $4 \mathrm{~d}$ theories $\mathscr{T}_{\mathbf{X}}^{4 \mathrm{~d}}$ we obtain from these singularities in IIB are all generalized ArgyresDouglas theories of type $\mathrm{AD}\left[G, G^{\prime}\right]$ (also known as $\mathrm{CNV}$ theories [67]), as indicated in table 2. We can also identify them as $D_{p}(\mathrm{SU}(N))$ trinions, following the discussion in the last section. 


\begin{tabular}{|c|c|c|c|c|c|c|c|c|c|c|c|c|}
\hline$E_{n, 0}^{(k)}$ & $4 \mathrm{~d} \mathrm{AD}$ & $F$ & $r$ & $f$ & $d_{\mathrm{H}}$ & $\widehat{r}$ & $\widehat{d}_{\mathrm{H}}$ & $a$ & $c$ & $\Delta \mathcal{A}_{r}$ & $b_{3}$ & $\mathfrak{f}$ \\
\hline$E_{6}^{(-1)}$ & {$\left[A_{2}, D_{4}\right]$} & $x_{1}^{3}+x_{2}^{3}+x_{3}^{3}+x_{4}^{2}$ & 0 & 0 & 4 & 4 & 0 & 2 & 2 & 0 & 0 & $\mathbb{Z}_{2}$ \\
\hline$E_{7}^{(-2)}$ & {$\left[A_{3}, A_{3}\right]$} & $x_{1}^{2}+x_{2}^{4}+x_{3}^{4}+x_{4}^{2}$ & 0 & 3 & 6 & 3 & 3 & $\frac{15}{8}$ & 2 & 0 & 0 & 0 \\
$E_{7}^{(-1)}$ & {$\left[A_{3}, E_{6}\right]$} & $x_{1}^{2}+x_{2}^{4}+x_{3}^{4}+x_{4}^{3}$ & 0 & 0 & 9 & 9 & 0 & 6 & 6 & 0 & 0 & $\mathbb{Z}_{3}$ \\
\hline$E_{8}^{(-4)}$ & {$\left[A_{2}, A_{5}\right]$} & $x_{1}^{2}+x_{2}^{3}+x_{3}^{6}+x_{4}^{2}$ & 0 & 2 & 6 & 4 & 2 & $\frac{9}{4}$ & $\frac{7}{3}$ & 0 & 0 & 0 \\
$E_{8}^{(-3)}$ & {$\left[A_{5}, D_{4}\right]$} & $x_{1}^{2}+x_{2}^{3}+x_{3}^{6}+x_{4}^{3}$ & 0 & 4 & 12 & 8 & 4 & $\frac{37}{6}$ & $\frac{19}{3}$ & 0 & 0 & 0 \\
$E_{8}^{(-2)}$ & {$\left[A_{5}, E_{6}\right]$} & $x_{1}^{2}+x_{2}^{3}+x_{3}^{6}+x_{4}^{4}$ & 0 & 2 & 16 & 14 & 2 & $\frac{49}{4}$ & $\frac{37}{3}$ & 0 & 0 & $\mathbb{Z}_{2}$ \\
$E_{8}^{(-1)}$ & {$\left[A_{5}, E_{8}\right]$} & $x_{1}^{2}+x_{2}^{3}+x_{3}^{6}+x_{4}^{5}$ & 0 & 0 & 20 & 20 & 0 & 20 & 20 & 0 & 0 & $\mathbb{Z}_{5}$ \\
\hline
\end{tabular}

Table 2. Terminal singularities ( $r=0$ models) of type $E_{n}^{(k)}$, with $k<0$, corresponding to $\left[G, G^{\prime}\right]$ $\mathrm{AD}$ theories as indicated. Note that $\Delta \mathcal{A}_{r} \equiv 24(c-a)-\widehat{d}_{\mathrm{H}}$.

The $4 \mathrm{~d}$ SCFT $\left[A_{2}, D_{4}\right]$ obtained from the singularity $E_{6}^{(-1)}$ is equivalent to a marginal gauging of three $D_{3}(\mathrm{SU}(2)) \cong\left[A_{1}, A_{3}\right] \mathrm{AD}$ theories with an $\mathrm{SU}(2)$ vector multiplet [36, 70]

$$
\mathscr{T}_{\mathbf{X}_{6}^{(-1)}}^{4 \mathrm{~d}}=D_{3} \underbrace{D_{3}}_{2} D_{3} .
$$

By the same token, the $E_{7}^{(k<0)}$ theories have the $4 \mathrm{~d}$ trinion description

$$
\mathscr{T}_{\mathbf{X}_{7}^{(-2)}}^{4 \mathrm{~d}}=D_{4} \underbrace{D_{2}}_{2} D_{4}, \quad \mathscr{T}_{E_{7}^{(-1)}}^{4 \mathrm{~d}}=D_{4} \underbrace{L_{2}}_{3} D_{4} .
$$

The $E_{8}^{(k<0)}$ models are similarly given by

$$
\begin{aligned}
& \mathscr{T}_{E_{8}^{(-4)}}^{4 \mathrm{~d}}= \\
& \left.D_{3}\right|_{2} ^{D_{2}} D_{6}, \\
& \mathscr{T}_{\mathbf{X}_{8}^{(-3)}}^{\mathrm{d}}=\quad D_{3} \underbrace{D_{2}}_{3} D_{6}, \\
& D_{2} \\
& \mathscr{T}_{\mathbf{X}_{8}^{(-2)}}^{4 \mathrm{~d}}=D_{3} \underbrace{D_{6}}_{4} D_{6}, \\
& \mathscr{T}_{E_{8}^{(-1)}}^{4 \mathrm{~d}}=\quad D_{3} \underbrace{D_{2}}_{5} D_{6} .
\end{aligned}
$$

Some of these models have a non-trivial one-form symmetry [36, 60], indicated by $\mathfrak{f}$ in table 2, which will play an important role below. Given the finite group $\mathfrak{f} \oplus \mathfrak{f}$, which can be computed from the geometry, one must choose a polarization in order to have a well-defined theory, which corresponds to a choice of allowed line operators [87, 88]. For simplicity, we will only consider purely 'electric' or 'magnetic' polarizations. For the 'electric' one-form symmetry, we can essentially think of the one-form symmetry of the trinions as (a subgroup 
of) the center symmetry of the central $\mathrm{SU}(N)$ node, in which case the charged objects are Wilson lines. Conversely, having a purely 'magnetic' one-form symmetry corresponds to choosing the central gauge group to be $\operatorname{PSU}(N)=\mathrm{SU}(N) / \mathbb{Z}_{N}$ (assuming $\mathfrak{f}=\mathbb{Z}_{N}$, for simplicity), in which case the charged objects are 't Hooft lines [89] that carry non-trivial $\mathbb{Z}_{N}$-valued magnetic charge, i.e. the 't Hooft lines specified by $\operatorname{PSU}(N)$ bundles on $S^{2}$ with a non-trivial Stiefel-Whitney class. In the following, we will denote $\mathrm{SU}(n) / \mathbb{Z}_{k}$ gauge groups by yellow nodes with the label $n / \mathbb{Z}_{k}$, as in (2.1). See also [50] a for a related recent discussion.

\subsection{Magnetic quivers and higher form symmetries}

We are particularly interested in the magnetic quiver $\mathrm{MQ}^{(5)}=\mathrm{EQ}^{(4)} / \mathrm{U}(1)^{f}$ for the $5 \mathrm{~d}$ SCFT $\mathcal{T}_{\mathbf{X}}^{5 \mathrm{~d}}$. Let us note that, if we start from a $4 \mathrm{~d}$ theory $\mathscr{T}_{\mathbf{X}}^{4 \mathrm{~d}}$ with an electric one-form symmetry $\mathbb{Z}_{N}$, we obtain a $3 \mathrm{~d}$ theory with a one-form symmetry, realized as a center symmetry of the $3 \mathrm{~d}$ quiver with central gauge group $\mathrm{SU}(N)$. On the other hand, if we start from $\mathscr{T}_{\mathbf{X}}^{4 \mathrm{~d}}$ with a magnetic one-form symmetry $\mathbb{Z}_{N}$, the $3 \mathrm{~d}$ quiver will have a $\mathbb{Z}_{N}$ zero-form magnetic symmetry. The charged operators are $3 \mathrm{~d}$ monopole operators with non-trivial $\mathbb{Z}_{N}$ magnetic charge, which descend from the 't Hooft lines of $\mathscr{T}_{\mathbf{X}}^{4 \mathrm{~d}}$ that wrap the $S^{1}$. By T-duality, the $3 \mathrm{~d}$ 'SU $(N)$ quiver' with an electric symmetry corresponds to the magnetic quiver of a $5 \mathrm{~d}$ SCFT $\mathcal{T}_{\mathbf{X}}^{5 \mathrm{~d}}$ with a 3 -form symmmetry. The 'electrically charged' objects in M-theory are M5-branes wrapping $S^{3} \times T^{2}$, with $T^{2}=S_{M}^{1} \times S_{\beta}^{1}$. These map to D3-branes wrapping $S^{3}$ in IIB. ${ }^{10}$ Conversely, the ' $\mathrm{SU}(N) / \mathbb{Z}_{N}$ ' theory with a magnetic symmetry corresponds to $\mathcal{T}_{\mathrm{X}}^{5 \mathrm{~d}}$ with a $\mathbb{Z}_{N}$ 0-form symmetry. The 'magnetically charged' objects are the M2-branes wrapping $S^{3}$, which map to D3-branes wrapping $S^{3} \times S_{\frac{1}{\beta}}^{1}$ in IIB; the latter objects are the $3 \mathrm{~d}$ monopole operators on the Coulomb branch of $\mathrm{MQ}^{(5)}$, which are dual to the M2-brane instantons on the Higgs branch of $\mathcal{T}_{\mathbf{X}}^{5 \mathrm{~d}}$.

As we will see in examples, whenever the $\mathrm{SU}(N) / \mathbb{Z}_{N}$ 'magnetic' theory is allowed by the geometry of the terminal singularity, the magnetic quiver is really equivalent to a unitary quiver, since $\operatorname{PSU}(N) \cong \mathrm{U}(N) / \mathrm{U}(1)$ and the overall $\mathrm{U}(1)$ of a unitary quiver is decoupled. It then turns out that these unitary quivers are ugly, and that repeated application of the GW duality shows that the $3 \mathrm{~d}$ theory consists only of $d_{\mathrm{H}}$ twisted hypermultiplets. Given this result, we can then obtain an explicit description of the magnetic quiver $\mathrm{MQ}^{(5)}$ for the 'electric' $5 \mathrm{~d}$ theory, which is obtained by gauging the $\mathbb{Z}_{N} 0$-form symmetry of the 'magnetic' theory. In the $3 \mathrm{~d} \mathcal{N}=4$ quiver description, the free twisted hypermultiplets are simply monopole operators of conformal dimension $\Delta=\frac{1}{2}$. The $\mathbb{Z}_{N}$ magnetic charge of a monopole operator can be computed as the total $\mathrm{U}(N)$ magnetic flux $\bmod N$, according to:

$$
w_{2}^{\mathrm{PSU}(N)} \cong c_{1}^{\mathrm{U}(N)} \quad \bmod N
$$

Therefore, the magnetic charge of the $\Delta=\frac{1}{2}$ monopole operators in the $\mathrm{U}(N)$ quiver determines the $\mathbb{Z}_{N}$ action of the 0 -form symmetry on the free twisted hypermultiplets. It

\footnotetext{
${ }^{10}$ It so happens that, for consistency with the $4 \mathrm{~d}$ gauge-theory language, we have to call these wrapped M5-branes 'electric' and the M2-brane 'magnetic'. This is only a matter of terminology and it should not cause any confusion.
} 
follows that the electric theory is equivalent to a discrete gauge theory of the form (3.1), with the $\mathbb{Z}_{N}$ charges determined as explained.

Let us now apply the above discussion to all the terminal singularities of table 2 .

\subsubsection{The $E_{6}$ model}

Consider first the $E_{6}^{(-1)}$ model, with the trinion description given by (3.2). Since $f=0$, the electric quiver of $\mathscr{T}_{\mathbf{X}}^{4 \mathrm{~d}}$ equals the magnetic quiver of $\mathcal{T}_{\mathbf{X}}^{5 \mathrm{~d}}$, and we have:

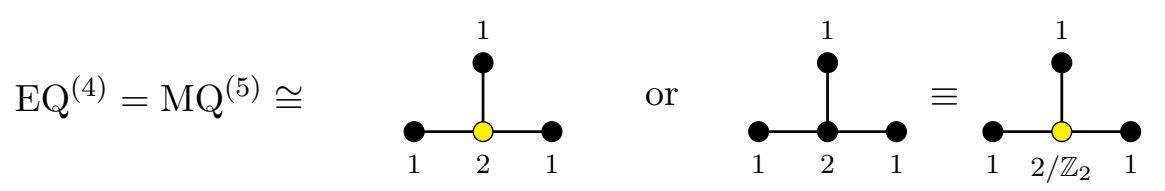

for the 'electric' or 'magnetic' version of $\mathscr{T}_{\mathbf{X}}^{4 \mathrm{~d}}$, respectively. ${ }^{11}$ As explained above, the quiver on the left in (3.6) is a $\mathbb{Z}_{2}$ gauging of the unitary quiver on the right. Conversely, we can obtain the quiver on the right by gauging the $\mathbb{Z}_{2}$ one-form symmetry of the ' $\mathrm{SU}(2)$ quiver' on the left. Note that the one-form symmetry is a center symmetry that exists as the diagonal $\mathbb{Z}_{2}$ between the $\mathbb{Z}_{2}$ center of $\mathrm{SU}(2)$ and $\mathbb{Z}_{2} \subset \mathrm{U}(1)$, which is preserved by the bifundamental matter.

The ' $\mathrm{U}(2)$ quiver' shown in (3.6) is ugly, since the central node is $\mathrm{U}(2)$ with $N_{f}=3$. By successive applications of the GW duality (2.33), we find that this theory is simply equivalent to four twisted hypermultiplets:

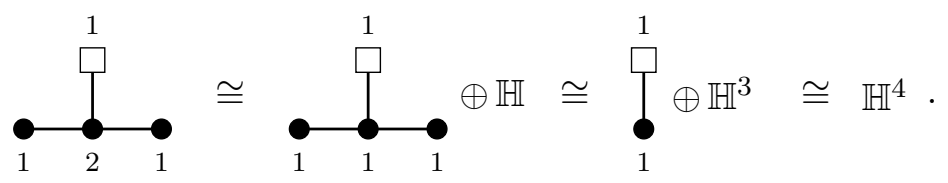

Let us now consider the $\mathrm{SU}(2)$ quiver, obtained as a $\mathbb{Z}_{2}$ gauging of the $\mathrm{U}(2)$ quiver. To keep track of the $\mathbb{Z}_{2}$ charge, consider the four monopole operators of the $U(2)$ quiver that flow to free twisted hypermultiplets (with $3 \mathrm{~d}$ conformal dimension $\Delta=\frac{1}{2}$ ) in the IR. It is easily checked that their magnetic charges under $\mathrm{U}(2) \times \mathrm{U}(1) \times \mathrm{U}(1)$, in the quiver notation of the left-hand-side of (3.7), are $^{12}$

$$
\left(\mathfrak{m}_{1}, \mathfrak{m}_{2} ; \mathfrak{n}^{(1)} ; \mathfrak{n}^{(2)}\right)=(1,0 ; 0 ; 0),(1,0 ; 1 ; 0),(1,0 ; 0 ; 1),(1,0 ; 1 ; 1) .
$$

The four operators have $\mathrm{U}(2)$ flux $(1,0)$, which means that they have the non-trivial charge under the $\mathbb{Z}_{2}$ magnetic symmetry. Therefore, we find that the 'SU(2) quiver' is a $\mathbb{Z}_{2}$ gauging of four hypermultiplets



\footnotetext{
${ }^{11}$ As mentioned above, in a quiver with only black nodes, the actual gauge group is $\prod_{i} \mathrm{U}\left(n_{i}\right) / \mathrm{U}(1)$, where the $\mathrm{U}(1)$ is the overall $\mathrm{U}(1)$. It is customary to write the 'all-black-dots' quiver as shown here. One can then 'ungauge' the $\mathrm{U}(1)$ factor at any gauge node and get an equivalent gauge theory.

${ }^{12}$ Note that we present the fluxes $\mathfrak{m}$ for the monopole operators in hypermultiplets, which are each comprised of two $3 \mathrm{~d} \mathcal{N}=2$ chiral-multiplet monopole operators with fluxes $\pm \mathfrak{m}$.
} 
with the $\mathbb{Z}_{2}$ action

$$
\mathbb{Z}_{2}: \mathbb{H}_{i} \rightarrow-\mathbb{H}_{i}, \quad i=1, \cdots, 4 .
$$

In particular, the $\mathrm{CB}$ of the quiver (3.9) and, therefore, the HB of the rank-0 5d SCFT, is given by the quotient $\mathbb{H}^{4} / \mathbb{Z}_{2}$. This quotient preserves the $\operatorname{Sp}(4)$ symmetry of $\mathbb{H}^{4}$, and therefore the Higgs branch of either the 'electric' or the 'magnetic' 5d SCFT has an Sp(4) symmetry. The Hilbert series of the CB of (3.9) was computed in [36] using the monopole formula [90]

$$
\begin{aligned}
\mathrm{HS}_{E_{6}^{(-1)}} & =\frac{1+28 t^{2}+70 t^{4}+28 t^{6}+t^{8}}{\left(1-t^{2}\right)^{8}} \\
& =\sum_{i=0}^{\infty} \operatorname{dim}([2 i, 0,0,0]) t^{2 i}=1+36 t^{2}+330 t^{4}+\mathcal{O}\left(t^{6}\right) .
\end{aligned}
$$

It is exactly the Hilbert Series of the minimal nilpotent orbit of $\mathfrak{s p}(4)$ [91]. This confirms that the flavor symmetry algebra is $\mathfrak{s p}(4)$,-the coefficient of $t^{2}$ being the number of conserved currents, $\operatorname{dim} \mathfrak{s p}(4)=36$. The appearance only of even spin representation of $\mathfrak{s p}(4)$ indicates in fact that the global form of the flavor symmetry is $\operatorname{Sp}(4) / \mathbb{Z}_{2}$.

Finally, let us mention that this model, at least in its 'magnetic' version, is equivalent to the $5 \mathrm{~d} T_{2}$ model [63] — in particular, the magnetic quiver on the right-hand-side of (3.6) makes the $\mathrm{SU}(2)^{3}$ symmetry of $T_{2}$ manifest. It is also the $\mathrm{MQ}^{(5)}$ one obtains for the $T_{2}$ toric geometry using the methods of [30]. We will generalize this observation to many more models below.

\subsubsection{The $E_{7}$ models}

The model $E_{7}^{(-2)}$ has $f=3$ and $\mathfrak{f}=0$. Using our general results for the 3 d reduction of $D_{p}(N)$, we find

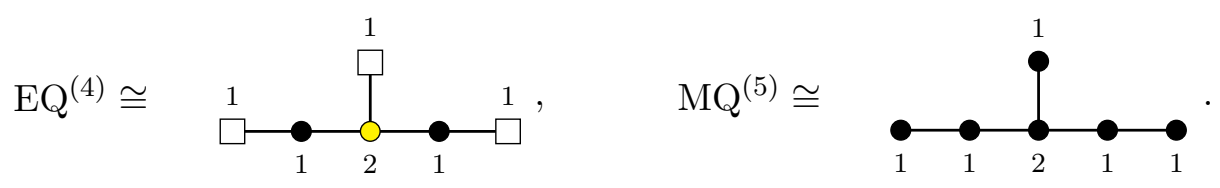

The absence of one-form symmetry is apparent both in $\mathrm{EQ}^{(4)}$, where the flavor at the center node breaks the $\mathbb{Z}_{2}$ center symmetry explicitly, and in $\mathrm{MQ}^{(5)}$. Here, $\mathrm{MQ}^{(5)}$ is again an ugly quiver, and one can check that it is equivalent to 6 twisted hypermultiplets. Thus, we find that the HB of the $5 \mathrm{~d} E_{7}^{(-2)}$ model is simply $\mathbb{H}^{6}$.

For the $E_{7}^{(-1)}$-type model, on the other hand, we have $f=0$ and $\mathfrak{f}=\mathbb{Z}_{3}$, and we find

$$
\mathrm{EQ}^{(4)}=\mathrm{MQ}^{(5)} \cong \bullet_{1}^{\bullet} \underbrace{1}_{3} \overbrace{2}^{1}
$$

for the 'electric' theory $\mathscr{T}_{\mathbf{X}}^{4 \mathrm{~d}}$ reduced on a circle. On the other hand the ' $\mathrm{SU}(3) / \mathbb{Z}_{3}$ quiver' is an ugly quiver, corresponding to replacing the central node in (3.13) by a black dot. That 
unitary quiver is equivalent to 9 twisted hypermultiplets. They arise from 9 monopole operators of dimensions $\Delta=\frac{1}{2}$, which carry the $\mathrm{U}(3)$ magnetic fluxes

$$
\left(\mathfrak{m}_{1}, \mathfrak{m}_{2}, \mathfrak{m}_{3}\right)=(1,0,0)^{9},
$$

where the exponent denote the number of times a given $\mathrm{U}(3)$ flux appears. Therefore, by gauging the $\mathbb{Z}_{3}$ magnetic symmetry, we see that the CB of (3.13) takes the form:

$$
\mathrm{CB}\left[\mathrm{MQ}^{(5)}\right] \cong \mathbb{H}^{9} / \mathbb{Z}_{3}, \quad \mathbb{Z}_{3}: \mathbb{H}_{i} \rightarrow \omega_{3} \mathbb{H}_{i}, \quad i=1, \cdots, 9,
$$

where $\omega_{n}$ denotes the $n$-th root of unity. This $\mathbb{Z}_{3}$ action preserves a subgroup $\mathrm{U}(9)$ of the flavor symmetry $\operatorname{Sp}(9)$, which is therefore the global symmetry of the 5 d 'electric' theory, consisting of a $\mathbb{Z}_{3}$ gauging of 9 hypermultiplets. The dimension of the global symmetry group can be checked by computing the first few terms of the Hilbert series of the CB of (3.13), which reads

$$
\mathrm{HS}_{E_{7}^{(-1)}}=1+81 t^{2}+330 t^{3}+2025 t^{4}+\mathcal{O}\left(t^{5}\right) .
$$

These coefficients can be decomposed in terms of the representations of $\mathfrak{s u}(9)$

$$
\begin{aligned}
\mathbf{8 1} & =\operatorname{dim}([1,0,0,0,0,0,0,1])+1 \\
\mathbf{3 3 0} & =\operatorname{dim}([3,0,0,0,0,0,0,0])+\operatorname{dim}([0,0,0,0,0,0,0,3]) \\
\mathbf{2 0 2 5} & =\operatorname{dim}([2,0,0,0,0,0,0,2])+\operatorname{dim}([1,0,0,0,0,0,0,1])+1 .
\end{aligned}
$$

None of these are charged under the center $\mathbb{Z}_{3} \subset \mathrm{U}(9)$, and hence we postulate that the global form of flavor symmetry in this case is $G_{F}=\mathrm{U}(9) / \mathbb{Z}_{3}$.

\subsubsection{The $E_{8}$ models}

The $E_{8}^{(-4)}$ model has $f=2$ and trivial higher-form symmetry. We then find

$$
\mathrm{EQ}^{(4)} \cong \overbrace{1}^{\prod_{2}}
$$

The quiver $\mathrm{MQ}^{(5)}$ is ugly and flows to six twisted hypermultiplets. Therefore, we find the 5d Higgs branch $\operatorname{HB}\left[E_{8}^{(-4)}\right] \cong \mathbb{H}^{6}$.

For the $E_{8}^{(-3)}$ model, which has $f=4$ and $\mathfrak{f}=0$, we similarly find

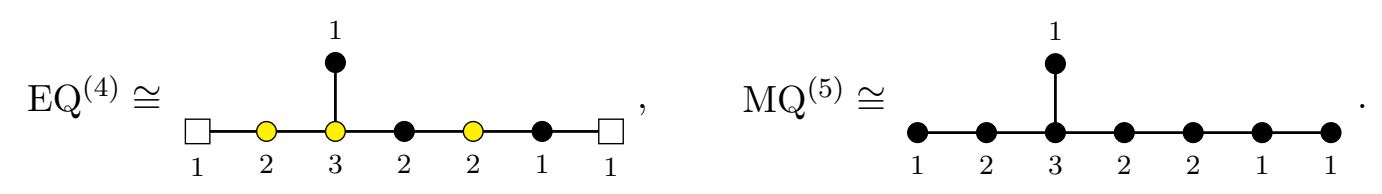

The magnetic quiver is ugly and equivalent to 12 twisted hypermultiplets, and therefore $\mathrm{HB}\left[E_{8}^{(-3)}\right] \cong \mathbb{H}^{12}$. 
The $E_{8}^{(-2)}$ model has $f=2$ and a non-trivial higher-form symmetry, $\mathfrak{f}=\mathbb{Z}_{2}$. This leads to
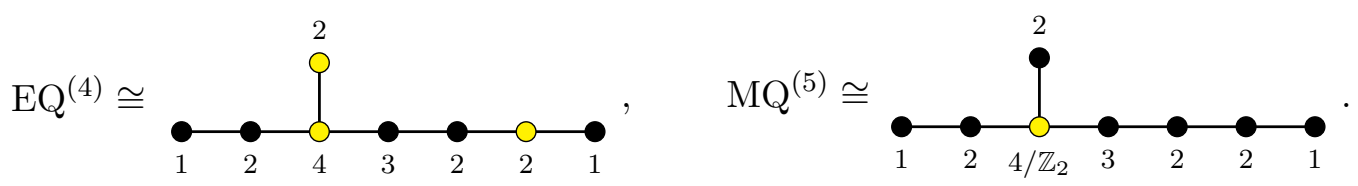

Here, the $\mathrm{EQ}^{(4)}$ is the $3 \mathrm{~d}$ reduction of the 'electric' $4 \mathrm{~d}$ SCFT $\mathscr{T}_{\mathbf{X}}^{4 \mathrm{~d}}$, which has a $\mathbb{Z}_{2}$ electric one-form symmetry; in the $3 \mathrm{~d}$ quiver, it corresponds to the diagonal $\mathbb{Z}_{2}$ center (common to the $\mathbb{Z}_{4}$ and $\mathbb{Z}_{2}$ center of the $\mathrm{SU}(4)$ and $\mathrm{SU}(2)$ gauge nodes, respectively). The magnetic quiver $\mathrm{MQ}^{(5)}$ similarly possesses a $\mathbb{Z}_{2}$ one-form symmetry, corresponding to the center of $\mathrm{SU}(4) / \mathbb{Z}_{2}$, which is inherited from the $\mathbb{Z}_{2} 3$-form symmetry of the 'electric' version of $\mathcal{T}_{\mathbf{X}}^{5 \mathrm{~d}}$.

The Higgs branch of the 'magnetic theory' in $5 \mathrm{~d}$ will instead be described by the quiver

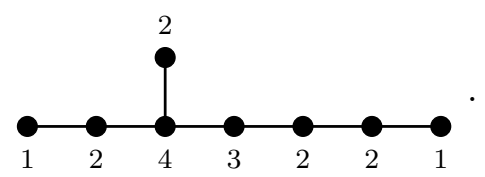

This is an ugly quiver which is equivalent to 16 twisted hypermultiplets. The $\Delta=\frac{1}{2}$ monopole operators of (3.21) have $\mathrm{U}(4)$ flux

$$
\left(\mathfrak{m}_{1}, \cdots, \mathfrak{m}_{4}\right)=(1,0,0,0)^{8},(1,1,0,0)^{6},(0,0,0,0)^{2} .
$$

The relevant $\mathbb{Z}_{2}$ charge is given by the total $\mathrm{U}(1)$ flux $\bmod 2$, and therefore we find

$$
\mathrm{CB}\left[\mathrm{MQ}^{(5)}\right] \cong\left(\mathbb{H}^{8} / \mathbb{Z}_{2}\right) \times \mathbb{H}^{8},
$$

where the $\mathbb{Z}_{2}$ acts by a sign on the first 8 hypermultiplets. We then conclude that the 'electric' version of $\mathcal{T}_{\mathbf{X}}^{5 \mathrm{~d}}$ has a global symmetry $\operatorname{Sp}(8) / \mathbb{Z}_{2} \times \operatorname{Sp}(8)$. The Coulomb branch Hilbert series of $\left[\mathrm{MQ}^{(5)}\right]$ from (3.20) reads

$$
\mathrm{HS}_{E_{8}^{(-2)}}=1+16 t+272 t^{2}+\mathcal{O}\left(t^{3}\right),
$$

in agreement with the above discussion. In particular, eight twisted hypermultiplets remain free, which give rise to the fundamental representation $\mathbf{1 6}$ of $\mathfrak{s p}(8)$, corresponding to the term $16 t$ in (3.24).

Finally, we consider the $E_{8}^{(-1)}$, which has $f=0$ and a $\mathbb{Z}_{5}$ higher-form symmetry. We thus find:

$$
\mathrm{EQ}^{(4)}=\mathrm{MQ}^{(5)} \cong \underbrace{}_{3}
$$

for the 'electric' 4d SCFT. Correspondingly, the $\mathrm{EQ}^{(4)}$ for the 'magnetic' theory is given by the same quiver with the $\mathrm{SU}(5)$ node replaced with a $\mathrm{U}(5)$ node:

$$
\mathrm{MQ}^{(5)} \cong \underbrace{}_{1}
$$


This latter quiver is ugly and equivalent to 20 twisted hypermultiplets. One can indeed find the 20 monopole operators of (3.26) with $\Delta=\frac{1}{2}$. They have $\mathrm{U}(5)$ flux:

$$
\left(\mathfrak{m}_{1}, \cdots, \mathfrak{m}_{5}\right)=(1,0,0,0,0)^{10},(1,1,0,0,0)^{10} .
$$

Therefore, the $\mathrm{CB}$ of the $\mathrm{MQ}^{(5)}(3.25)$, and the $\mathrm{HB}$ of $\mathcal{T}_{\mathbf{X}}^{5 \mathrm{~d}}$, take the form:

$$
\mathrm{CB}\left[\mathrm{MQ}^{(5)}\right] \cong \mathbb{H}^{20} / \mathbb{Z}_{5}
$$

with the action:

$$
\mathbb{Z}_{5}:\left(\mathbb{H}_{i}, \mathbb{H}_{j}\right) \rightarrow\left(\omega_{5} \mathbb{H}_{i}, \omega_{5}^{2} \mathbb{H}_{j}\right), \quad i=1, \cdots, 10, \quad j=1, \cdots, 10 .
$$

This action preserves a $(\mathrm{U}(10) \times \mathrm{U}(10)) / \mathbb{Z}_{5}$ subgroup of $\operatorname{Sp}(20)$. The Hilbert series is

$$
\mathrm{HS}_{E_{8}^{(-1)}}=1+200 t^{2}+\mathcal{O}\left(t^{3}\right)
$$

and confirms the dimension of the symmetry group of the 'magnetic' version of $\mathcal{T}_{\mathbf{X}}^{5 \mathrm{~d}}$.

In summary, for this small family of terminal singularities, the Higgs branch of the rank-zero $\mathcal{T}_{\mathbf{X}}^{5 \mathrm{~d}}$ is given by

$$
\mathrm{HB}\left[\mathcal{T}_{\mathrm{X}}^{5 \mathrm{~d}}\right] \cong \mathbb{H}^{d_{\mathrm{H}} / \mathfrak{f}} \quad \text { or } \quad \mathbb{H}^{d_{\mathrm{H}}}
$$

for the 'electric' or 'magnetic' version of the theory, respectively, with the $\mathfrak{f}=\mathbb{Z}_{k}$ actions specified above. Note that the 'electric' theory with a non-trivial $\mathfrak{f}$ has a 3 -form symmetry $\Gamma^{(3)}=\mathfrak{f}$, as expected for a discrete gauging of free hypermultiplets in $5 \mathrm{~d}[36,88]$.

\section{Generalized $E_{n}$ theories and discrete gauging}

In this section, we study the $E_{n}^{(k)}$ singularities introduced in (2.59), for $k \geq 0$. They give rise to three infinite families of $5 \mathrm{~d}$ SCFTs, $\mathcal{T}_{\mathbf{X}}^{5 \mathrm{~d}}=E_{n}^{(k)}, n=6,7,8$, of arbitrary rank $r$, with

$$
r=\left\lfloor\frac{k}{\Delta_{n}}\right\rfloor+1, \quad \text { with } \quad \Delta_{6}=3, \Delta_{7}=4, \Delta_{8}=6 .
$$

Here, the dimension $\Delta_{n}$ is the conformal dimension of the CB operator of the rank-one $4 \mathrm{~d}$ $\mathcal{N}=2$ Minahan-Nemeschanski (MN) theory with $E_{n}$ flavor symmetry [92, 93]. For $k=0$, the $E_{n}^{(k)}$ canonical singularity precisely gives rise to the rank-one $E_{n} 5 \mathrm{~d}$ SCFT, which flows to the $4 \mathrm{~d} E_{n} \mathrm{MN}$ theory upon compactification on a circle. For $\frac{k}{\Delta_{n}}=\ell \in \mathbb{Z}$, one engineers the higher-rank $E_{n}$ theories (with $r=\ell+1$ ). Finally, for $\frac{k}{\Delta_{n}}$ not an integer, we will argue that we simply obtain the $E_{n}$ theories tensored with free hypermultiplets, modulo some discrete gauge group.

As we argued in section 2.4 , the $4 \mathrm{~d} \operatorname{SCFT} \mathscr{T}_{\mathbf{X}}^{4 \mathrm{~d}}$ is a trinion with central node $\mathrm{SU}(N)$, with $N=\Delta_{n}+k$. We therefore find [56]

$$
\begin{aligned}
& \mathscr{T}_{\mathbf{X}_{6}^{(k)}}^{4 \mathrm{~d}}=D_{3} \underbrace{D_{3}}_{N=3+k} D_{3}, \quad \mathscr{T}_{E_{7}^{(k)}}^{4 \mathrm{~d}}=D_{4} \underbrace{D_{2}}_{N=4+k} D_{4}, \\
& \mathscr{T}_{E_{8}^{(k)}}^{4 \mathrm{~d}}=D_{3} \underset{N=6+k}{D_{2}} D_{6} .
\end{aligned}
$$




\begin{tabular}{|c|c|c|c|c|c|c|c|c|c|c|c|}
\hline$E_{n}^{(k)}$ & $F$ & $r$ & $f$ & $d_{\mathrm{H}}$ & $\widehat{r}$ & $\widehat{d}_{\mathrm{H}}$ & $a$ & $c$ & $\Delta \mathcal{A}_{r}$ & $b_{3}$ & $\mathfrak{f}$ \\
\hline$E_{6}$ & $x_{1}^{3}+x_{2}^{3}+x_{3}^{3}+x_{4}^{3}$ & 1 & 6 & 11 & 5 & 7 & $\frac{109}{24}$ & $\frac{29}{6}$ & 0 & 0 & 0 \\
$E_{6}^{(1)}$ & $x_{1}^{3}+x_{2}^{3}+x_{3}^{3}+x_{4}^{4}$ & 1 & 0 & 12 & 12 & 1 & 10 & 10 & -1 & 2 & $\mathbb{Z}_{4}$ \\
$E_{6}^{(2)}$ & $x_{1}^{3}+x_{2}^{3}+x_{3}^{3}+x_{4}^{5}$ & 1 & 0 & 16 & 16 & 1 & 16 & 16 & -1 & 2 & $\mathbb{Z}_{5}$ \\
\hline$E_{7}$ & $x_{1}^{2}+x_{2}^{4}+x_{3}^{4}+x_{4}^{4}$ & 1 & 7 & 17 & 10 & 8 & $\frac{31}{3}$ & $\frac{32}{3}$ & 0 & 0 & 0 \\
$E_{7}^{(1)}$ & $x_{1}^{2}+x_{2}^{4}+x_{3}^{4}+x_{4}^{5}$ & 1 & 0 & 18 & 18 & 1 & 18 & 18 & -1 & 2 & $\mathbb{Z}_{5}$ \\
$E_{7}^{(2)}$ & $x_{1}^{2}+x_{2}^{4}+x_{3}^{4}+x_{4}^{6}$ & 1 & 3 & 24 & 21 & 4 & $\frac{207}{8}$ & 26 & -1 & 2 & $\mathbb{Z}_{3}$ \\
$E_{7}^{(3)}$ & $x_{1}^{2}+x_{2}^{4}+x_{3}^{4}+x_{4}^{7}$ & 1 & 0 & 27 & 27 & 1 & 36 & 36 & -1 & 2 & $\mathbb{Z}_{7}$ \\
\hline$E_{8}$ & $x_{1}^{2}+x_{2}^{3}+x_{3}^{6}+x_{4}^{6}$ & 1 & 8 & 29 & 21 & 9 & $\frac{225}{8}$ & $\frac{57}{2}$ & 0 & 0 & 0 \\
$E_{8}^{(1)}$ & $x_{1}^{2}+x_{2}^{3}+x_{3}^{6}+x_{4}^{7}$ & 1 & 0 & 30 & 30 & 1 & 40 & 40 & -1 & 2 & $\mathbb{Z}_{7}$ \\
$E_{8}^{(2)}$ & $x_{1}^{2}+x_{2}^{3}+x_{3}^{6}+x_{4}^{8}$ & 1 & 2 & 36 & 34 & 3 & $\frac{209}{4}$ & $\frac{157}{3}$ & -1 & 2 & $\mathbb{Z}_{4}$ \\
$E_{8}^{(3)}$ & $x_{1}^{2}+x_{2}^{3}+x_{3}^{6}+x_{4}^{9}$ & 1 & 4 & 42 & 38 & 5 & $\frac{397}{6}$ & $\frac{199}{3}$ & -1 & 2 & $\mathbb{Z}_{3}$ \\
$E_{8}^{(4)}$ & $x_{1}^{2}+x_{2}^{3}+x_{3}^{6}+x_{4}^{10}$ & 1 & 2 & 46 & 44 & 3 & $\frac{329}{4}$ & $\frac{247}{3}$ & -1 & 2 & $\mathbb{Z}_{5}$ \\
$E_{8}^{(5)}$ & $x_{1}^{2}+x_{2}^{3}+x_{3}^{6}+x_{4}^{11}$ & 1 & 0 & 50 & 50 & 1 & 100 & 100 & -1 & 2 & $\mathbb{Z}_{11}$ \\
\hline
\end{tabular}

Table 3. The $r=1 E_{n}^{(k)}$ models.

For $N=m \Delta_{n}$, the $4 \mathrm{~d}$ theory is Lagrangian, see the quivers in (2.63).

\subsection{The rank-one $E_{n}^{(k)}$ theories and their magnetic quivers}

Let us first consider the $r=1 E_{n}^{(k)}$ models, with $k=0, \cdots, \Delta_{n}-1$; their basic geometric data is summarized in table 3 . For $k=0$, these models engineer the rank-one $E_{n} 5 \mathrm{~d}$ SCFTs. Indeed, these singularities admit crepant resolutions with a single exceptional divisor. The resolved singularity $\widetilde{\mathbf{X}}$ takes the form:

$$
\begin{array}{ll}
E_{6}^{(k)}: & x_{1}^{3}+x_{2}^{3}+x_{3}^{3}+\delta_{1}^{k} x_{4}^{3+k}, \\
E_{7}^{(k)}: & x_{1}^{2}+x_{2}^{4}+x_{3}^{4}+\delta_{1}^{k} x_{4}^{4+k}, \\
E_{8}^{(k)}: & x_{1}^{2}+x_{2}^{3}+x_{3}^{6}+\delta_{1}^{k} x_{4}^{6+k} .
\end{array}
$$

For $k=0$, the exceptional divisor $\left\{\delta_{1}=0\right\}$ is a smooth del Pezzo surface, $d P_{n}$. For $k>0$, the exceptional locus is a singular surface:

$$
\begin{array}{lll}
E_{6}^{(k)}: & \delta_{1}=0:\left\{x_{1}^{3}+x_{2}^{3}+x_{3}^{3}=0\right\} \subset \mathbb{P}^{3}, \\
E_{7}^{(k)}: & \delta_{1}=0:\left\{x_{1}^{2}+x_{2}^{4}+x_{3}^{4}=0\right\} \subset \mathbb{P}_{[2,1,1,1]}^{3}, \\
E_{8}^{(k)}: & \delta_{1}=0:\left\{x_{1}^{2}+x_{2}^{3}+x_{3}^{6}=0\right\} \subset \mathbb{P}_{[3,2,1,1]}^{3} .
\end{array}
$$

In addition, for $k>1$, we have a residual terminal singularity at $\delta_{1}=x_{1}=x_{2}=x_{3}=0$ (on the patch $x_{4}=1$ ). These terminal singularities are of the type studied in the previous section: the $r=1$ model $E_{n}^{(k)}$ with $k>1$ contains the terminal singularity $E_{n}^{\left(k-\Delta_{n}\right)}$. 


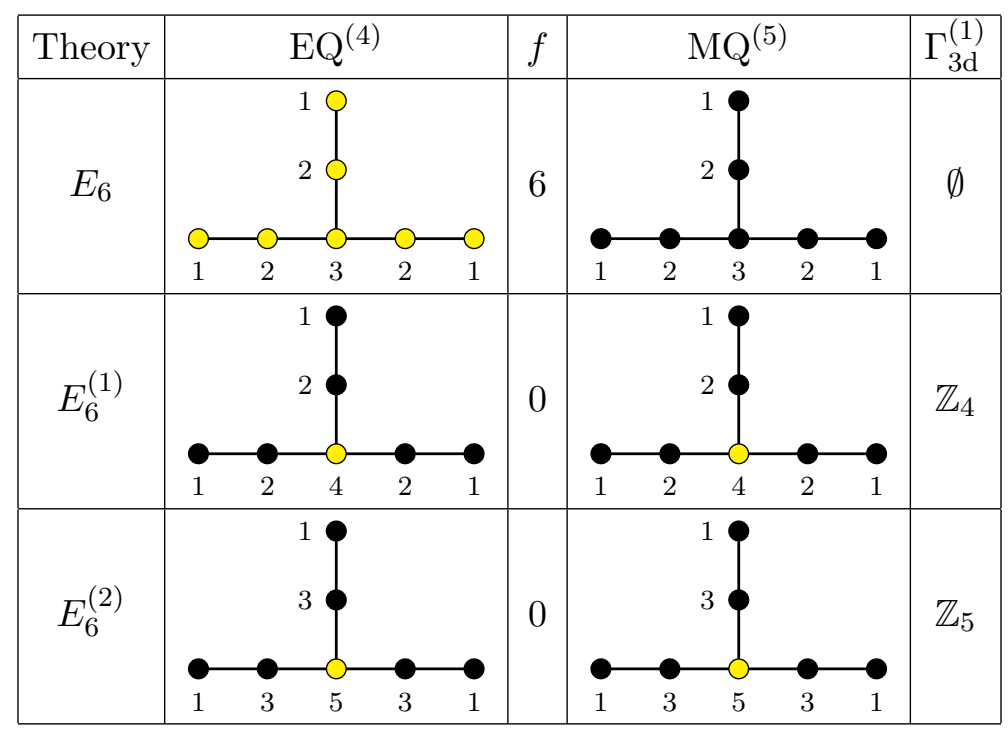

Table 4. $\mathrm{EQ}^{(4)}$ and $\mathrm{MQ}^{(5)}$ for the $r=1 E_{6}$ theories. Here we present the 'electric' ('SU $(N)$ quiver') version of each theory, which has a one-form symmetry $\Gamma_{3 \mathrm{~d}}^{(1)}=\mathfrak{f}$ in $3 \mathrm{~d}$.

As we see from table 3, these model display an interesting pattern of higher-form symmetries when $k>0$, since $\mathfrak{f}$ is non-trivial. As in the previous section, we should distinguish between the 'electric'/SU(N)-type theory and 'magnetic'/U(N)-type theories.

We argued above that the terminal singularities $E_{n}^{(k<0)}$ give rise to free 5 d hypermultiplets in M-theory, in the 'magnetic' version of the theory. Therefore, from the above pattern of crepant resolutions, we expect that the $r=1 E_{n}^{(k>1)} 5 \mathrm{~d}$ SCFTs have a Coulombbranch low-energy theory that contains additional free hypermultiplets. By studying the Higgs branch of these models through their magnetic quivers, we will further argue that these free hypermultiplets actually arise as decoupled free sectors.

The quiverines $\mathrm{EQ}^{(4)}$ and $\mathrm{MQ}^{(5)}$ are given in tables 4, 5 and 6, in their 'electric' versions. With that choice of global structure, the electric 1-form symmetry of $\mathscr{T}_{\mathbf{X}}^{4 \mathrm{~d}}$ maps to a one-form symmetry of the $3 \mathrm{~d}$ quiver, $\Gamma_{3 \mathrm{~d}}^{(1)}=\mathfrak{f}$; correspondingly, the $5 \mathrm{~d}$ theory $\mathcal{T}_{\mathbf{X}}^{5 \mathrm{~d}}$ has a 3 -form symmetry $\Gamma_{5 d}^{(3)}=\mathfrak{f}$.

On the other hand, the 'magnetic' version of the magnetic quivers $\mathrm{MQ}^{(5)}$, corresponding to having a five-dimensional one-form symmetry $\Gamma_{5 \mathrm{~d}}^{(0)}=\mathfrak{f}$, are given by the same quivers with only black nodes (only $\mathrm{U}(n)$ gauge groups, modulo an overall $\mathrm{U}(1)$ ). These magnetic quivers are shown in tables 7, 8 and 9 below, for general $k$.

\subsubsection{The $r=1 E_{6}^{(k)}$ theories}

Let us first consider the $E_{6}$ series in detail. The $\mathrm{MQ}^{(5)}$ for $E_{6} \equiv E_{6}^{(0)}$ in table 4 is the wellknown magnetic quiver for the $E_{6}$ theory, in the shape of the affine $E_{6}$ Dynkin diagram; its $3 \mathrm{~d} \mathcal{N}=4$ Coulomb branch is the one- $E_{6}$-instanton moduli space, of dimension $d_{\mathrm{H}}=11$. Note that is was crucial to gauge the $\mathrm{U}(1)^{6}$ flavor symmetry of $\mathrm{EQ}^{(4)}$ to obtain $\mathrm{MQ}^{(5)}$, as explained in $[36,37]$; the resulting $\mathrm{U}(1)_{T}^{6}$ topological symmetry becomes the Cartan of the $E_{6} 3 \mathrm{~d}$ CB flavor symmetry. 
The $E_{6}^{(1)}$ model has $r=1$ and $d_{\mathrm{H}}=12$. The $\mathrm{U}(N)$ version of the $\mathrm{MQ}^{(5)}$ is given by

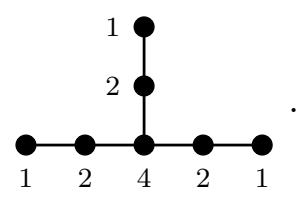

Note that the central node is 'bad' in the GW sense, since it is locally U(4) SQCD with $N_{f}=6$ flavors. Correspondingly, the $3 \mathrm{~d}$ UV description contains monopole operators of vanishing dimension. Therefore, this quiver description does not give a direct handle on the IR theory in $3 \mathrm{~d}$.

In this case, one can check that the 'badness' of the quiver (4.5) only comes from its central node. Consider $\mathrm{U}(N)$ SQCD with $N_{f}=2 N-2$ flavors. It is known that the most singular locus on the Coulomb branch of that theory is $\mathbb{H} \cong \mathbb{C}^{2}$, and that along that locus the low-energy effective theory is $\mathrm{U}(N-1)$ SQCD with $N_{f}=2 N-2$, plus a free twisted hypermultiplet whose vacuum expectation value $(\mathrm{VEV})$ parametrizes the $\mathbb{C}^{2}$ locus [62]. ${ }^{13}$ This is the effect of a non-perturbative Higgs mechanism:

$$
\mathrm{U}(N) \rightarrow \mathrm{U}(N-1) \times \mathrm{U}(1),
$$

as we tune the CB parameters to the most singular locus. At the central node of the quiver, we locally have such a situation, with $N=4$. We then propose that the relevant moduli space of the $E_{6}^{(1)}$ magnetic quiverine is obtained by zooming in onto that most singular locus. By effectively replacing the $\mathrm{U}(4)$ node by an $\mathrm{U}(3)$ node in (4.5), the relevant $3 \mathrm{~d} \mathrm{CB}$ of $\mathrm{MQ}^{(5)}$ is then obtained from the $3 \mathrm{~d}$ theory:

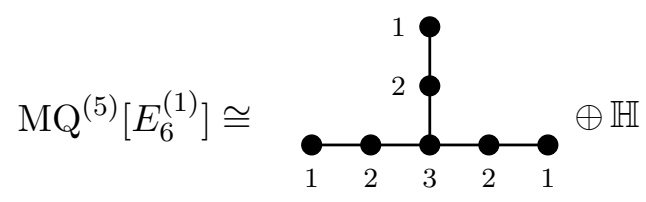

which is the $\mathrm{MQ}^{(5)}$ of the rank-1 $E_{6}$ theory plus a decoupled (twisted) hypermultiplet.

This interpretation is corroborated by looking at the Higgs branch of the $\mathrm{MQ}^{(5)}$, which describes the Coulomb branch of $\mathcal{T}_{\mathbf{X}}^{5 \mathrm{~d}}$ compactfied on a torus. Both descriptions (4.5) and (4.7) give the same result: the $\mathrm{HB}$ of $\mathrm{MQ}^{(5)}$ is given by $\mathbb{C}^{2} / \Gamma_{E_{6}}$, the Kleinian singularity of type $E_{6}$, plus a free twisted hypermultiplet. Indeed, it is well-known that the HB of the quiver with $\mathrm{U}(3)$ central node is $\mathbb{C}^{2} / \Gamma_{E_{6}}$, and one can check that the same is true for the quiver (4.5) (using, for instance, the methods of [96]). In the latter case, however, there is a residual 'unhiggsed' $U(1)$ vector multiplet at low-energy on the HB, which gives us the free twisted hypermultiplet in the IR. This simple structure of the HB of $\mathrm{MQ}^{(5)}$ is also consistent with the structure of the smooth resolved threefold $\widetilde{\mathbf{X}}_{E_{6}^{(1)}}$. Importantly, the resolved threefold contains a pair of three-cycles $\left(b_{3}=2\right)$, which, from the M-theory perspective, give rise to a free (uncharged) hypermultiplet on the $5 \mathrm{~d}$ Coulomb branch, in perfect agreement with the above $\mathrm{MQ}^{(5)}$.

\footnotetext{
${ }^{13}$ This Seiberg-like duality was first suggested by [94], and its meaning was clarified in [62]. See also [95].
} 


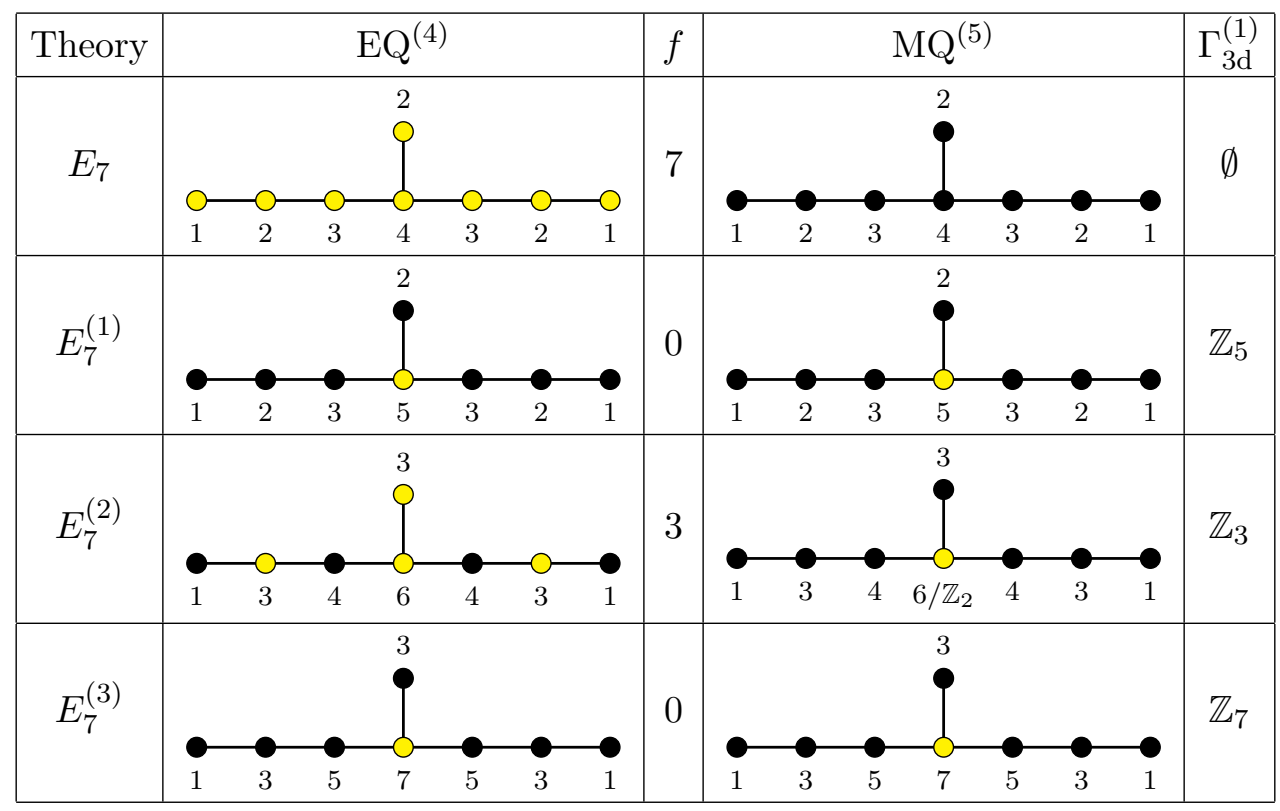

Table 5. $\mathrm{EQ}^{(4)}$ and $\mathrm{MQ}^{(5)}$ for the $r=1 E_{7}$ theories and their 1-form symmetry.

These considerations lead us to conjecture that the 'magnetic' version of the $E_{6}^{(1)} 5 \mathrm{~d}$ SCFT is simply the $E_{6}$ SCFT tensored with a free hypermultiplet:

$$
\left.\mathcal{T}_{\mathbf{X}_{E_{6}^{(1)}}^{5 \mathrm{~d}}}\right|_{\text {'magnetic' }} \cong \mathcal{T}_{\mathbf{X}_{E_{6}}^{5 \mathrm{~d}}} \otimes \mathbb{H}
$$

We will present further evidence for this claim in section 5 .

The asymptotic geometry of the canonical singularity tells us that the $5 \mathrm{~d}$ theory (4.8) has a distinguished $\mathbb{Z}_{4}$ 0-form symmetry. By gauging it, we expect to find the 'electric' version of the theory, with a $\mathbb{Z}_{4} 3$-form symmetry in $5 \mathrm{~d}$ :

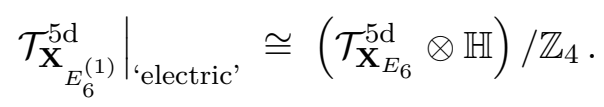

The $\mathbb{Z}_{4}$ action used in (4.9) can be understood at the level of the quantum Higgs branch. Indeed, the $\mathrm{MQ}^{(5)}$ for the 'electric' $E_{6}^{(1)}$ is the one with $\mathrm{SU}(4)$ central node shown in table 4 , which is a $\mathbb{Z}_{4}$ gauging of the unitary quiver (4.5).

The $E_{6}^{(2)}$ model has $r=1$ and $d_{\mathrm{H}}=16$. Now, the resolved geometry both contains a 3 -cycle and a terminal singularity of type $E_{6}^{(-1)}$. The physics of this model is again easiest to understand in its 'magnetic' version. The unitary $\mathrm{MQ}^{(5)}$ has a U(5) central node with $N_{f}=9$ flavors, so that one can dualize it to a U(4) node plus a free hypermultiplet. At this stage, the $\mathrm{U}(3)$ nodes on the three legs become ugly, and can each be dualized:

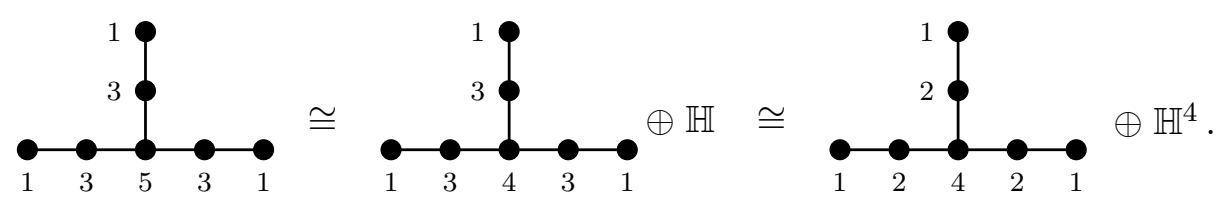


We then obtain the $\mathrm{MQ}^{(5)}$ of the $E_{6}^{(1)}$ theory plus four twisted hypermultiplets. We claim that this free sector is associated to the terminal singularity $E_{6}^{(-1)}$ that arises on the resolved threefold $\widetilde{\mathbf{X}}_{E_{6}^{(2)}}$. We then treat the $E_{6}^{(1)}$ factor as above, and conclude that the 'magnetic' version of the $E_{6}^{(2)}$ theory in $5 \mathrm{~d}$ is equivalent to the $E_{6}$ theory tensored with five free hypermultiplets. We can summarize this decomposition pictorially as
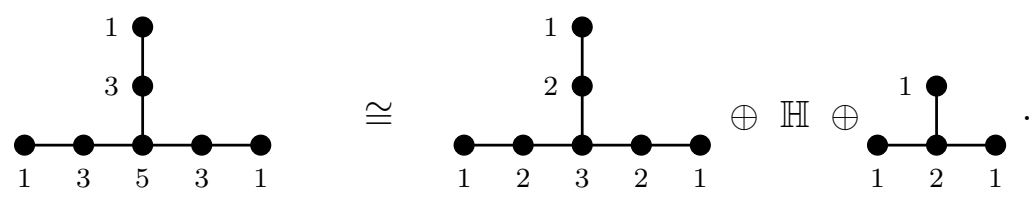

This pattern is again consistent with the analysis of the Higgs branch for the $\mathrm{MQ}^{(5)}$ on the left of (4.11), which is still $\mathbb{C}^{2} / \Gamma_{E_{6}}$ together with a larger unhiggsable sector that is equivalent to the $E_{6}^{(-1)} \cong T_{2}$ magnetic quiver (plus a free twisted hypermultiplet) on the right of (4.11). We also note, heuristically, that this result can be obtained by a naive application of the rules of quiver substraction [46, 97].

Finally, the 'electric version' of the theory is obtained from the 'magnetic' version by gauging the $\mathbb{Z}_{5}$ 0-form symmetry. In conclusion, we find

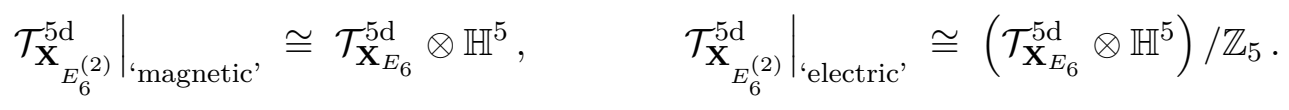

\subsubsection{The $r=1 E_{7}^{(k)}$ and $E_{8}^{(k)}$ theories}

The $r=1 E_{7}$ and $E_{8}$ series are completely similar. The magnetic quivers with 3 d 1-form symmetries are shown in tables 5 and 6 . For $k=1$, the central node is again 'bad' with a balance $e \equiv N_{f}-2 N_{c}=-2$, and we should go to the most singular locus on the CB, where we obtain the effective description

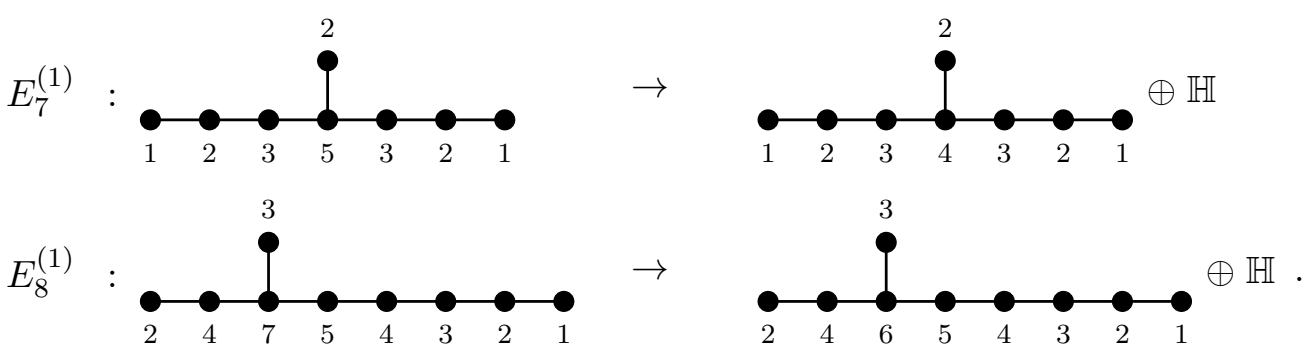

We then argue that the $5 \mathrm{~d}$ SCFT $E_{n}^{(1)}$ with a non-trivial 0-form symmetry is given by

$$
E_{n}^{(1)} \cong E_{n} \otimes \mathbb{H}
$$

for the same reasons as before. Similarly, for $k>1$, the unitary magnetic quivers can be reduced to the case $E_{n}^{(1)}$ by repeated application of the GW duality on ugly nodes. We then find

$$
\mathrm{MQ}^{(5)}\left[E_{n}^{(k)}\right] \cong \mathrm{MQ}^{(5)}\left[E_{n}\right] \oplus \mathbb{H} \oplus \mathrm{MQ}^{(5)}\left[E_{n}^{\left(k-\Delta_{n}\right)}\right]
$$

Therefore, the 'magnetic' version of the $r=1 E_{n}^{(k)}$ SCFT $\mathcal{T}_{\mathbf{X}}^{5 \mathrm{~d}}$ consists of the $E_{n}$ SCFT tensored with the appropriate number of free hypermultiplets. The electric version is then obtained by gauging $\Gamma_{5 \mathrm{~d}}^{(0)}=\mathfrak{f}$. 




Table 6. $\mathrm{EQ}^{(4)}$ and $\mathrm{MQ}^{(5)}$ for the $r=1 E_{8}$ series of theories and their 1-form symmetry.

\subsection{The higher-rank $E_{n}^{(k)}$ theories}

Consider the $E_{n}^{(k)}$ singularities with:

$$
k=\Delta_{n} \ell+\widehat{k}, \quad \widehat{k}=0, \cdots, \Delta_{n}-1,
$$

which give rise to $5 \mathrm{~d}$ SCFTs of rank $r=\ell+1$. For the resolution geometry, see appendix A for more details.

The analysis of the $4 \mathrm{~d}$ SCFTs $\mathscr{T}_{\mathbf{X}}^{4 \mathrm{~d}}$ and of the electric quiverines $\mathrm{EQ}^{(4)}$ is similar to the $\ell=0$ case. Here, we will mainly discuss the magnetic quivers $\mathrm{MQ}^{(5)}$ with unitary gauge groups, for the 'magnetic version' of the theory. They are displayed in tables 7, 8 and 9. As before, the 'electric version' is obtained by discretely gauging the $5 \mathrm{~d}$ 0-form symmetry.

Let us first consider the case $\widehat{k}=0$. The magnetic quivers are given by:


for the $E_{6}, E_{7}$ and $E_{8}$ theories, respectively, with $m \equiv \ell+1$. The Higgs branch of this $3 \mathrm{~d}$ $\mathcal{N}=4$ quiver is $\operatorname{Sym}^{m}\left(\mathbb{C}^{2} / \Gamma_{E_{n}}\right)$, and its low-energy theory also contains $\ell=m-1$ free 


\begin{tabular}{|c|c|c|}
\hline$k$ & $\mathrm{MQ}^{(5)} \& \boldsymbol{\lambda} \& \boldsymbol{\lambda}^{\prime}$ & $\Gamma_{5 \mathrm{~d}}^{(0)}=\mathfrak{f}$ \\
\hline $3 \ell+0$ & $\underset{\left\{[\ell+1, \ell+1, \ell+1]^{3}\right\}}{\ell+1}$ & $\mathbb{Z}_{\ell+1}$ \\
\hline $3 \ell+1$ & $\left\{\begin{array}{c}2 \ell+2 \\
\left\{[\ell+2, \ell+1, \ell+1]^{3}\right\}\end{array}\right.$ & $\mathbb{Z}_{3 \ell+4}$ \\
\hline $3 \ell+2$ & $\underbrace{\ell+1}_{\left\{[\ell+2, \ell+2, \ell+1]^{3}\right\}}$ & $\mathbb{Z}_{3 \ell+5}$ \\
\hline
\end{tabular}

Table 7. Magnetic quivers for the $E_{6}^{(k)}$ models. The three partitions $\lambda$ are partitions of $N \equiv \Delta_{n}+k$ associated with the three legs of the quiver. The second partition (if listed) is the one after the box-lifting, later denoted by $\boldsymbol{\lambda}^{\prime}$. These are introduced here for later reference.

twisted hypermultiplets. Correspondingly, we have $\ell$ free hypermultiplets on the Coulomb branch of $\mathcal{T}_{\mathrm{X}}^{5 \mathrm{~d}}$, which arise in the resolved geometry from the $\ell$ pairs of 3-cycles associated to the $\ell$ exceptional divisors that are ruled surfaces of genus 1 .

Importantly, although each node is individually balanced, the quivers (4.17) are nonetheless 'bad', since they have monopole operators of dimension 0 . These $\Delta=0$ monopole operators are precisely related to the unhigssable $\mathrm{U}(1)^{\ell}$ factors. These quivers have been studied from various point of view in the literature [66]. We will discuss them further in section 5 .

Next, we consider the case $\widehat{k}>0$. We can easily see that the quivers with $\widehat{k} \geq 2$ are equivalent to the $\widehat{k}=1$ quivers by successive dualization of the 'ugly' nodes. For instance, the $E_{6}^{(3 \ell+2)}$ quiver can be reduced to the $E_{6}^{(3 \ell+1)}$ quiver exactly as in the $\ell=0$ case (4.10). The resulting free sector is the one associated with the residual terminal singularity $E_{n}^{\left(\widehat{k}-\Delta_{n}\right)}$, for any $\ell \geq 0$.

We therefore only need to understand the $\widehat{k}=1$ models. In that case and for any $\ell \geq 0$, the central node is bad with a balance $e=-2$. By the same argument as before, we claim that the relevant physics occurs on the most singular locus of the $\mathrm{CB}$, whose effective 


\begin{tabular}{|c|c|c|}
\hline$k$ & $\mathrm{MQ}^{(5)} \& \boldsymbol{\lambda} \& \boldsymbol{\lambda}^{\prime}$ & $\Gamma_{5 \mathrm{~d}}^{(0)}=\mathfrak{f}$ \\
\hline $3 \ell+0$ & 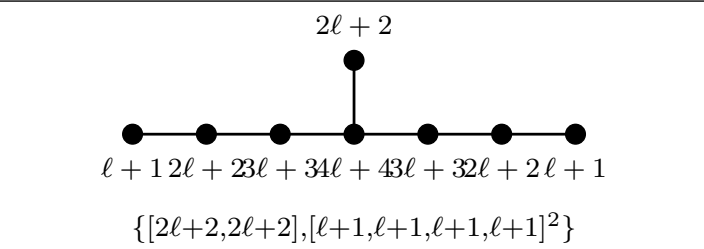 & $\mathbb{Z}_{\ell+1}$ \\
\hline $4 \ell+1$ & $\begin{array}{l}\ell+12 \ell+23 \ell+34 \ell+53 \ell+32 \ell+2 \ell+1 \\
\left\{[2 \ell+3,2 \ell+2][\ell+2, \ell+1, \ell+1, \ell+1]^{2}\right\} \\
\left.\{2 \ell+3,2 \ell+2]\left[(\ell+2)(\ell+1)^{3}\right]\left[(\ell+1)^{4}, 1\right]\right\}\end{array}$ & $\mathbb{Z}_{4 \ell+5}$ \\
\hline $4 \ell+2$ & $\left\{[2 \ell+3,2 \ell+3][(\ell+2)(\ell+1)(\ell+2)(\ell+1)]\left[(\ell+2),(\ell+1)^{3}, 1\right]\right\}$ & $\mathbb{Z}_{2 \ell+3}$ \\
\hline $4 \ell+3$ & $\left\{\begin{array}{l}\ell+12 \ell+23 \ell+54 \ell+73 \ell+52 \ell+3 \ell+1 \\
\left\{[2 \ell+4,2 \ell+3][\ell+2, \ell+2, \ell+2, \ell+1]^{2}\right\}\end{array}\right.$ & $\mathbb{Z}_{4 \ell+7}$ \\
\hline
\end{tabular}

Table 8. Magnetic quivers for the $E_{7}^{(k)}$ models. The three partitions $\boldsymbol{\lambda}$ are partitions of $N \equiv \Delta_{n}+k$ associated with the three legs of the quiver. The second partition (if listed) is the one after the box-lifting, later denoted by $\boldsymbol{\lambda}^{\prime}$. These are introduced here for later reference.

description is given by

$$
\mathrm{MQ}\left[E_{n}^{\left(\Delta_{n} \ell+1\right)}\right] \rightarrow \operatorname{MQ}\left[E_{n}^{\left(\Delta_{n} \ell\right)}\right] \oplus \mathbb{H} .
$$

The magnetic quiver $\mathrm{MQ}\left[E_{n}^{\left(\Delta_{n} \ell+1\right)}\right]$ is given in (4.17).

In conclusion, we see that the 'magnetic' 5 d theory is always a rank- $(\ell+1) E_{n}$ theory tensored with free hypermultiplets, while the 'electric' theory is necessarily a discrete gauging thereof:

$$
\left.\mathcal{T}_{\mathbf{X}_{E_{n}}^{(k)}}^{5 \mathrm{~d}}\right|_{\text {'magnetic' }} \cong \mathcal{T}_{\mathbf{X}_{E_{n}}}^{5 \mathrm{~d}} \otimes \mathbb{H}^{d_{\mathrm{H}}(\widehat{k})+1},\left.\quad \mathcal{T}_{\mathbf{X}_{E_{n}^{(k)}}^{5 \mathrm{~d}}}^{\mathrm{d}}\right|_{\text {'electric' }} \cong\left(\mathcal{T}_{\mathbf{X}_{E_{n}}}^{5 \mathrm{~d}} \otimes \mathbb{H}^{d_{\mathbf{H}}(\widehat{k})+1}\right) / \mathfrak{f}
$$

Here we have $k=\Delta_{n} \ell+\widehat{k}$, the integer $d_{\mathrm{H}}(\widehat{k})$ is defined as the dimension of the $5 \mathrm{~d}$ HB of the rank-zero theory $E_{n}^{\left(\widehat{k}-\Delta_{n}\right)}$ for $\widehat{k}>1$, and by $d_{\mathrm{H}}(\widehat{k})=0$ if $\widehat{k}=1$; finally, the discrete group $\mathfrak{f}$ is given for any $E_{n}^{(k)}$ in the tables 7,8 and 9 . 


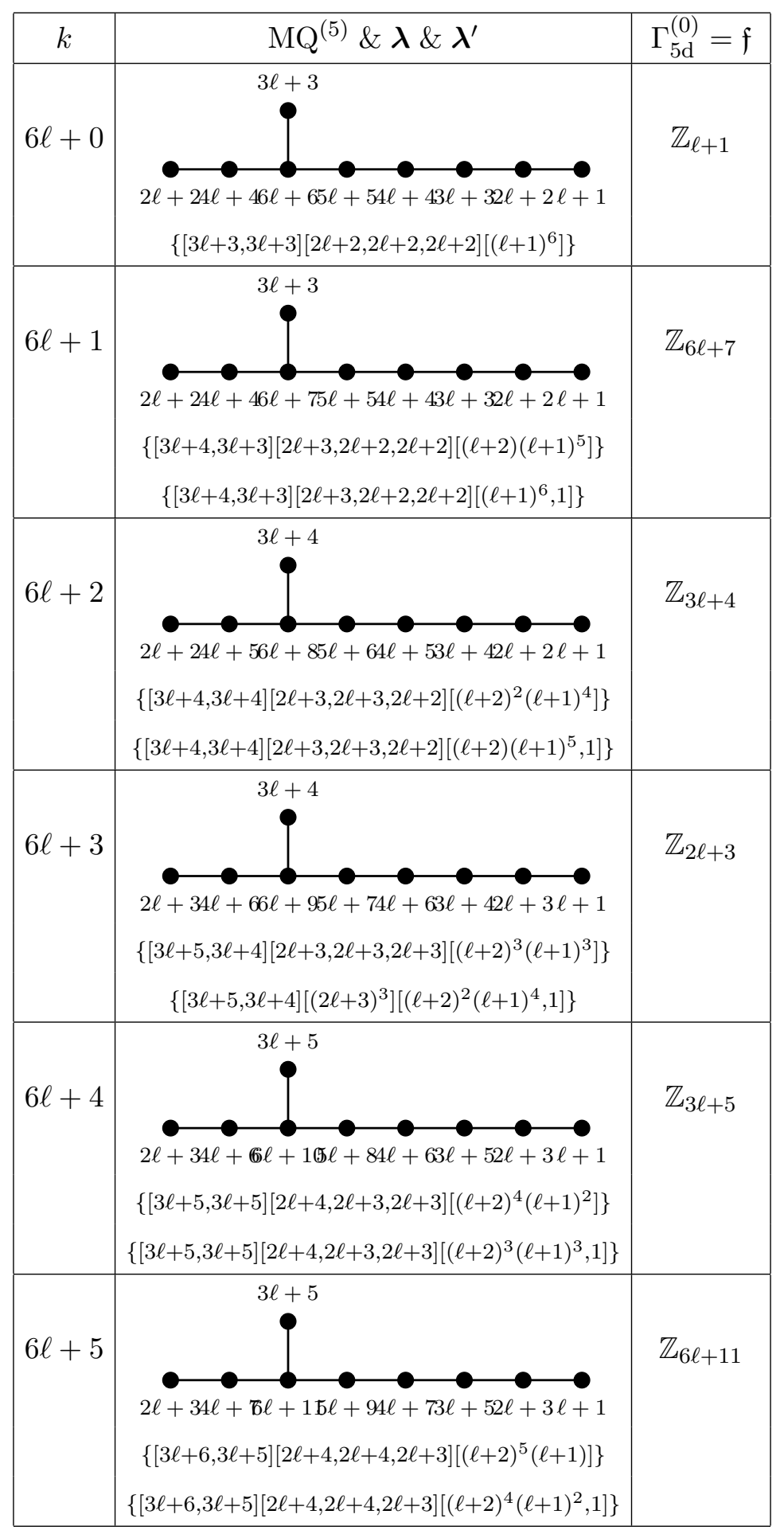

Table 9. Magnetic quivers for the $E_{8}^{(k)}$ models. We list the partitions $\boldsymbol{\lambda}$ that underlies these models, the one after the box-lifting $\boldsymbol{\lambda}^{\prime}$, as well as the 0 -form symmetry of the associated 5d SCFT. 
It is also interesting to note that, for the canonical singularities $\mathbf{X}_{E_{n}^{(k)}}$, the residual terminal singularities in the crepant resolution $\widetilde{\mathbf{X}}$ are related to the 'local ugliness' of the magnetic quiver, while the 3-cycles in the crepant resolution are associated with the 'badness' of the magnetic quiver for $E_{n}^{(1)}$ obtained after getting rid of all the ugly nodes (i.e. with balance $e=-1$ ) by repeated dualization.

\section{$5 \quad$ Class $\mathcal{S}$ description, brane-webs and partial higgsing}

Let $\mathcal{S}_{N, \boldsymbol{\lambda}}^{4 \mathrm{~d}}$ denote a $4 \mathrm{~d} \mathcal{N}=2 \mathrm{SCFT}$ of class $\mathcal{S}$ [64], defined as the $A_{N-1} 6 \mathrm{~d} \mathcal{N}=(0,2)$ theory with three regular punctures, indexed by three partitions of $N$ :

$$
\boldsymbol{\lambda} \equiv\left\{\lambda^{(\alpha)}\right\}_{\alpha=1}^{3}=\left\{\left[\lambda_{1}^{(1)}, \lambda_{2}^{(1)}, \cdots\right]\left[\lambda_{1}^{(2)}, \lambda_{2}^{(2)}, \cdots\right]\left[\lambda_{1}^{(3)}, \lambda_{2}^{(3)}, \cdots\right]\right\} .
$$

We propose that our $5 \mathrm{~d}$ SCFTs $\mathcal{T}_{\mathrm{X}_{E_{n}^{(k)}}^{5}}^{\mathrm{d}}$ compactified on a circle give rise to such a class $\mathcal{S}$ theory in the IR:

$$
\left.D_{S^{1}} \mathcal{T}_{\mathbf{X}_{E_{n}^{(k)}}^{5 \mathrm{~d}}}\right|_{\mathrm{IR}} \longleftrightarrow \mathcal{S}_{N, \boldsymbol{\lambda}}^{4 \mathrm{~d}}, \quad \quad N=\Delta_{n}+k .
$$

The main piece of evidence for this conjecture is that the magnetic quivers for the $5 \mathrm{~d}$ $\mathrm{SCFT} E_{n}^{(k)}$ are identical to the magnetic quivers (also known as $3 \mathrm{~d}$ mirrors) of these class $\mathcal{S}$ theories [65, 98]. For $k=0$, the duality (5.2) is the well-studied relation between the $5 \mathrm{~d}$ rank-one $E_{n}$ SCFTs and the $4 \mathrm{~d} E_{n}$ Minahan-Nemeschansky (MN)-theories. In this section, we explore the generalization to any $k$, which sheds some additional light on this family of models. We expect (5.2) to be true at least for the 'magnetic' version of $\mathcal{T}_{\mathrm{X}}^{5 \mathrm{~d}}$ with (in general) a non-trivial 0 -form symmetry $\Gamma_{5 \mathrm{~d}}^{(0)}=\mathfrak{f}$. Identifying (and gauging) that 0 -form symmetry in the class $\mathcal{S}$ description is left for future work.

The partitions $\boldsymbol{\lambda}$ are obtained from the three unitary linear quivers that form the three tails of $\mathrm{MQ}^{(5)}$, which correspond to the $3 \mathrm{~d} T_{\lambda}[\mathrm{SU}(N)]$ theories [61]. They are written down explicitly in tables 7, 8, and 9. Each leg consists of a quiver $[N]-\mathrm{U}\left(n_{1}\right)-\mathrm{U}\left(n_{2}\right) \cdots$ attached to the central $\mathrm{U}(N)$, and it determines the partition $\lambda=\left[\lambda_{a}\right]$ of $N$ according to $\lambda_{a}=n_{a-1}-n_{a}$, with $n_{0} \equiv N$. Such partitions obtained from our $\mathrm{MQ}^{(5)}$ are not all ordered when $1<\widehat{k}<\Delta_{n}-1$. This is due to the ugliness of some of the tails. A GW dualization at the ugly nodes along the tails gives us properly ordered partitions (and a few twisted hypermultiplets).

Such trinion class $\mathcal{S}$ theories, also called fixtures [65], can be uplifted to 5 d theories described by type-IIB $(p, q)$-webs [99, 100], corresponding to a junctions of three stacks of $N$ D5-branes, $N$ NS5-branes and $N(1,1) 5$-branes, respectively, with the three stacks of fivebranes ending on 7-branes with boundary conditions specified by the partitions $\lambda^{(\alpha)}[63] .{ }^{14}$ We then expect this brane-web construction to be dual to our canonical singularity in M-theory:

$$
\mathcal{T}_{\mathbf{X}_{E_{n}}^{(k)}}^{5 \mathrm{~d}} \longleftrightarrow \quad \text { trinion-shaped brane web in IIB } \longleftrightarrow \text { triangle-shaped GTP }
$$

\footnotetext{
${ }^{14}$ Recall that, locally, each stack of 5-branes can be T-dualized (and S-dualized) to the D3-brane boundary condition described by the $3 \mathrm{~d} \mathcal{N}=4 T_{\lambda}[\mathrm{SU}(N)]$ theory. This is how the magnetic quiver is most easily understood, in this setup.
} 
with the last description to be discussed momentarily. In other words, the duality (5.2) uplifts to $5 \mathrm{~d}$. In the special case when all every external 5 -brane ends on its own 7 -brane, this is the well-known duality between the $T_{N} \cong \mathbb{C}^{3} /\left(\mathbb{Z}_{N} \times \mathbb{Z}_{N}\right)$ toric geometry and 5-brane webs $[63,101]$. The general case is a conjecture, based on a fair amount of circumstantial evidence.

These branes webs can be efficiently represented as dot diagrams [63], also known as a generalized toric polygons (GTP) [30,35]. In particular, given a GTP, there is a systematic algorithm (either based on brane-web technology [46, 97] or directly in the GTP [30]) for computing the magnetic quiver of the SCFT defined by the brane web/GTP, whenever that magnetic quiver is 'good' or 'ugly' [30,35]. In the present setup, all GTP are triangles related to the $T_{N}$ theory. The partitions $\boldsymbol{\lambda}$ give the successive spacing lengths between black dots along the three edges of length $N$, starting from the lower-left corner and going counter-clockwise.

\subsection{Rank-zero trinions}

To illustrate the class $\mathcal{S} /$ GTP perspective (and our notation), let us first consider the rank-zero $5 \mathrm{~d}$ models of section 3 . As already pointed out, the $E_{6}^{(-1)}$ model is the $T_{2}$ theory, whose corresponding GTP reads

$$
E_{6}^{(-1)}=T_{2}:
$$

The $E_{7}^{(-2)}$ magnetic quiver appearing in (3.12) has two ugly legs, and it is then equivalent to $T_{2}$ plus two hypermultiplets. The $E_{7}^{(-1)}$ model, on the other hand, is given by the trinion

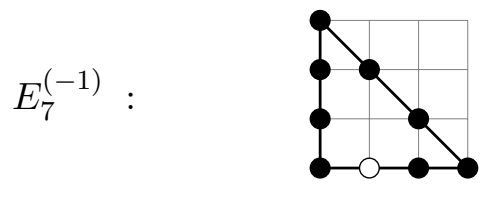

$$
\boldsymbol{\lambda}=\left\{[2,1][1,1,1]^{2}\right\} .
$$

This describes 9 hypermultiplets, and corresponds to the second $A_{2}$ free-field fixture in [65]. Similarly, for the $E_{8}$ models, we have $E_{8}^{(-4)} \cong T_{2} \oplus \mathbb{H}^{2}$ and $E_{8}^{(-3)} \cong E_{7}^{(-1)} \oplus \mathbb{H}^{3}$ after dualizing the ugly legs. We also find

$$
E_{8}^{(-2)}:
$$

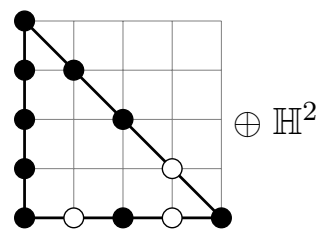

$$
\boldsymbol{\lambda}=\{[2,2][2,1,1][1,1,1,1]\}
$$

Here, we first dualize the ugly nodes on the legs of $\mathrm{MQ}^{(5)}$, which gives us the two hypermultiplets. We are then left with the $A_{4}$ free-field fixture corresponding to 14 hypermulti- 
plets [65]. Finally, we have

$$
E_{8}^{(-1)}:
$$

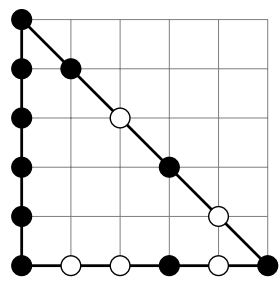

$$
\boldsymbol{\lambda}=\{[3,2][2,2,1][1,1,1,1,1]\}
$$

This is the $A_{5}$ free-field fixture corresponding to 20 hypermultiplets [65].

\subsection{Coulomb branch spectrum and 'badness' in the class $\mathcal{S}$ description}

The class $\mathcal{S}$ description leads to a complementary point of view on the potential 'badness' of the magnetic quiver. Recall that the Coulomb branch operators of dimension $\Delta \in \mathbb{Z}$ of a $4 \mathrm{~d} \mathcal{N}=2$ SCFT of class $\mathcal{S}$ correspond to $\Delta$-differentials on the Gaiotto curve - in our case, on the 3-punctured sphere - with poles at the punctures specified by some integers $p_{\Delta}^{(\alpha)}$. The virtual number of operators of dimension $\Delta$, denoted by $d_{\Delta}$, is given by the Riemann-Roch theorem [64, 65]

$$
d_{\Delta}=h^{0}\left(L_{\Delta}\right)-h^{1}\left(L_{\Delta}\right)=1-2 \Delta+\sum_{\alpha=1}^{3} p_{\Delta}^{(\alpha)}
$$

with $L_{\Delta}=\mathcal{K}^{\Delta}\left(p_{\Delta}^{(\alpha)}\right)$. Here, we specialized to the three-punctured sphere. The integers $p_{\Delta}$ associated to a given (regular) puncture are obtained as follows. Let us draw the transposed Young tableau associated to the partition $\lambda$, and let us start numbering the boxes from the upper-left corner, with the first box labeled by $p_{1} \equiv 0$, the second box to the right labelled by $p_{2}=1$, and so forth from left to right and downwards; moreover, each time we go to the next row, we start the new row with the same value of $p$ as in the last box on the previous row. This defines a vector $p_{\Delta}$, with $\Delta=2, \cdots, N .^{15}$

In particular, (5.8) gives a formula for the 'virtual rank' of the class $\mathcal{S}$ theory:

$$
\left.r\right|_{\text {virtual }}=\sum_{\Delta=2}^{N} d_{\Delta}
$$

This equals the rank, $r$, if and only if the virtual dimension $d_{\Delta}$ equal the actual dimension, $d_{\Delta}=h^{0}\left(L_{\Delta}\right)$, for all $\Delta$. Using the relation

$$
\sum_{\Delta=2}^{N} p_{\Delta}=\frac{N(N-1)}{2}-\frac{1}{2} \sum_{a} \lambda_{a}\left(\lambda_{a}-1\right),
$$

which holds for any partition of $N, \lambda=\left[\lambda_{a}\right]$, we find the general expression for the virtual rank of our trinion theories:

$$
\left.r\right|_{\text {virtual }}=\frac{(N-1)(N-2)}{2}-\frac{1}{2} \sum_{\alpha=1}^{3} \sum_{a} \lambda_{a}^{(\alpha)}\left(\lambda_{a}^{(\alpha)}-1\right) .
$$

This matches the rank computed from the GTP using the generalised s-rule [30, 35].

\footnotetext{
${ }^{15}$ For instance, for the partitions of $N=5$ appearing in (5.7), we have: $[3,2] \rightarrow$\begin{tabular}{l|l|l|}
\hline & 1 \\
\hline & 2 & 2 \\
\hline 2
\end{tabular}$: p_{\Delta}=(1,1,2,2)$,

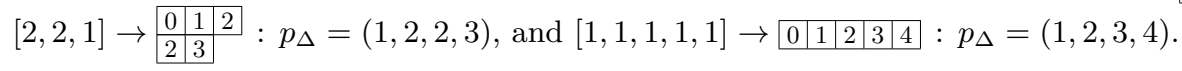


In general, however, some $d_{\Delta}$ could be negative, in which case the virtual rank is smaller than the true rank. For all the $E_{n}^{(k)}$ theories, we find that $d_{\Delta} \in\{1,0,-1\}, \forall \Delta$. We then propose the following heuristic formula for the actual rank:

$$
r=\sum_{\Delta \mid d_{\Delta}>0} d_{\Delta}
$$

It turns out that the number of $\Delta$ 's such that $d_{\Delta}=-1$ is precisely equal to $\frac{1}{2} b_{3}$, the number of pairs of 3-cycles in the resolved threefold $\widetilde{\mathbf{X}}$. Indeed, $d_{\Delta}=-1$ really means that $h^{1}\left(L_{\Delta}\right)=1$, which should be interpreted as an additional degree of freedom on the Coulomb branch of the class $\mathcal{S}$ theory. Since these 3-cycles give rise to free hypermultiplets on the Coulomb branch of $\mathcal{T}_{\mathbf{X}}^{5}$, we propose that these $\mathcal{K}$-valued $\Delta$-differential on the Gaiotto curve should also correspond to free hypermultiplets on the CB of the class $\mathcal{S}$ theory $\mathcal{S}_{N, \lambda}^{4 \mathrm{~d}}$.

As an example of the above discussion, consider the $r=1 E_{6}^{(k)}$ models. The corresponding GTPs and partitions are

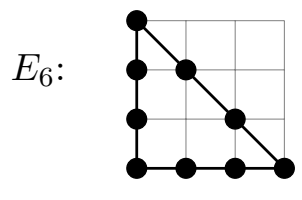

$\boldsymbol{\lambda}=\left\{[1,1,1]^{3}\right\}$

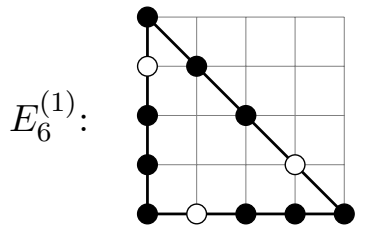

$\boldsymbol{\lambda}=\left\{[2,1,1]^{3}\right\}$

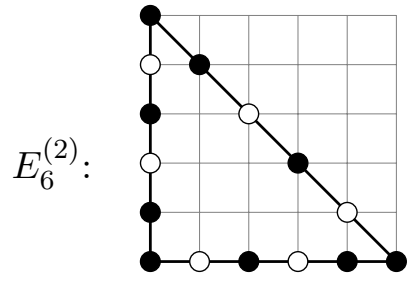

$\boldsymbol{\lambda}=\left\{[2,2,1]^{3}\right\}$.

The vectors of virtual dimensions are then

$$
d_{\Delta}=(0,1), \quad d_{\Delta}=(0,1,-1), \quad d_{\Delta}=(0,1,-1,0),
$$

respectively. The first model is the $E_{6} \mathrm{MN}$-theory, with a single $\mathrm{CB}$ operator of dimension $\Delta=3$. The other two models also have a CB operator of dimension 3 , but there is also a single negative entry at $\Delta=4$, so that their virtual rank vanishes.

The higher-rank theories are also interesting. For the $r=2 E_{6}$ theory, we have

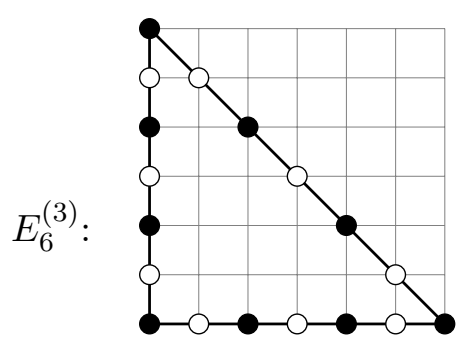

$\boldsymbol{\lambda}=\left\{[2,2,2]^{3}\right\}$,

$d_{\Delta}=(0,1,-1,0,1)$,

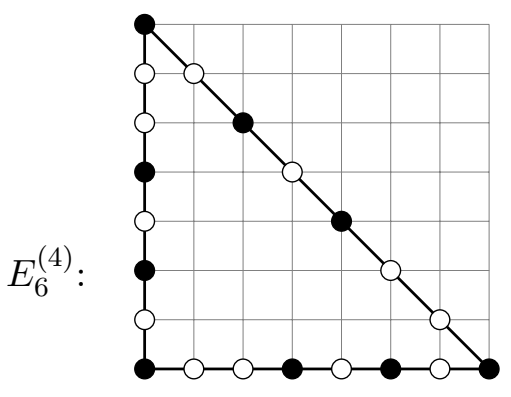

$\boldsymbol{\lambda}=\left\{[3,2,2]^{3}\right\}$,

$d_{\Delta}=(0,1,-1,0,1,-1)$,

and also $d_{\Delta}=(0,1,-1,0,1,-1,0)$ for $E_{6}^{(5)}$. The rank-2 $E_{6}$ theory, $E_{6}^{(3)}$, indeed has rank 2 according to the formula (5.12). We also have one $d_{\Delta}=-1$ at $\Delta=4$, in agreement with 
$b_{3}=2$. Similarly, for $E_{6}^{(4)}$, we have two -1 entries in $d_{\Delta}$, corresponding to $b_{3}=4$ in the geometry.

This discussion readily generalizes to all the $E_{n}^{(k)}$ trinions with $k=\Delta_{n} \ell+\widehat{k}$. We have

$$
\begin{array}{llll}
d_{\Delta}=1 & \text { for } & \Delta=(j+1) \Delta_{n}, & j=0, \cdots, \ell, \\
d_{\Delta}=-1 & \text { for } & \Delta=(j+1) \Delta_{n}+1, & j=0, \cdots, \ell-\delta_{\widehat{k}, 0} .
\end{array}
$$

The central node of the $\mathrm{MQ}^{(5)}$ is bad whenever $d_{N}=-1$, which corresponds to $\widehat{k}=1$. For a fixed $\ell$, the vector $d_{\Delta}$ of the models with $\widehat{k}>1$ is the same as for the model with $\widehat{k}=1$, with $\widehat{k}-1$ zeros appended.

\subsection{Redeeming bad theories by box-lifting}

Up to this point, we have seen the badness of our $E_{n}^{(k)}$ theories for $k>0$ appear in at least three complementary ways: from the magnetic quivers (which describes the HB of $\mathcal{T}_{\mathbf{X}}^{5 \mathrm{~d}}$ ), from the presence of three-cycles in the resolved geometry $\widetilde{\mathbf{X}}$ (which engineers the $\mathrm{CB}$ of $\mathcal{T}_{\mathbf{X}}^{5 \mathrm{~d}}$ ), and from the virtual dimensions of $\Delta$-differentials on the Gaiotto curve (which engineers the $\mathrm{CB}$ of $\mathcal{S}_{N, \lambda}^{4 \mathrm{~d}}$ ).

In section 4, we have also argued that we could redeem part of the badness of the magnetic quiver - essentially, for $k=1$ - by considering a special locus of the CB of the bad $\mathrm{MQ}^{(5)}$. Here, we would like to further discuss how we may cure the bad magnetic quivers more generally, using the class $\mathcal{S}$ intuition. This discussion closely follows the proposal of [66] on how to cure the higher-rank $E_{n}$ theories. See also [102] for a related discussion from the Higgs-branch point of view.

The basic idea is to embed the bad theory, $\mathcal{S}$, into a larger 'good' theory, $\mathcal{S}^{\prime}$, so that we can go back from $\mathcal{S}^{\prime}$ to $\mathcal{S}$ by partial Higgsing (that is, by taking a particular limit onto the HB of $\mathcal{S}^{\prime}$ ). Indeed, any trinion $\mathcal{S}_{N, \boldsymbol{\lambda}}^{4 \mathrm{~d}}$ can be obtained by partially closing the punctures (or, equivalently, by lifting boxes in the partition) of the $T_{N}$ theory, which is the theory corresponding to three full punctures, $\boldsymbol{\lambda}=\left\{\left[1^{N}\right]^{3}\right\}$. The GTP of $T_{N}$ is a triangle of length $N$ with only black dots, and any reduction of the punctures correspond to adding white dots along the edges.

This embedding $\mathcal{S}_{N, \boldsymbol{\lambda}}^{4 \mathrm{~d}} \subset \mathcal{S}^{\prime}$ implicitly defines the bad theory. In favorables cases, we can then confirm that the original 'bad' theory is indeed equivalent to a (higher-rank) $E_{n}$ theory tensored with a free sector. In terms of the partitions $\boldsymbol{\lambda}$ defining $\mathcal{S}_{N, \boldsymbol{\lambda}}^{4 \mathrm{~d}}$, the proposal of [66] was to consider $\mathcal{S}^{\prime}$ defined by the partitions obtained by 'lifting up one box' of one Young tableaux associated to $\boldsymbol{\lambda}$. In terms of the GTP, this corresponds to 'filling in' a white dot. We will see concrete examples of this in the following.

\subsubsection{Rank-one $E_{n}^{(k)}$ theories and GTPs}

Let us again consider the simplest bad theory in detail. This is the $E_{6}^{(1)}$ theory, whose GTP is shown in (5.13). We propose to uplift this theory to the class $\mathcal{S}$ theory defined by 
the partitions

$$
E_{6}^{(1)^{\prime}}: \quad \lambda^{\prime}=\{[2,1,1][2,1,1][1,1,1,1]\}:
$$

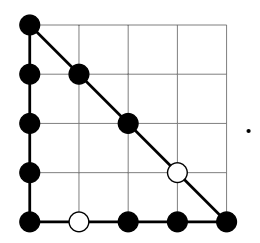

This is a good theory, in particular, the virtual dimensions $d_{\Delta}$ in this case are $d_{\Delta}=(0,1,0)$. This theory can be higgsed back to $E_{6}^{(1)}$ by partially closing the full puncture, $\lambda=[1,1,1,1]$, to $[2,1,1]$. Physically, this corresponds to activating a minimal nilpotent VEV for the moment map of the SU(4) global symmetry associated with the full puncture. Such a higgsing can lead to different theories in the IR, depending on how it is performed, as we will now explain.

The trinion (5.17) describes the rank-1 $E_{6}$ theory plus four free hypermultiplets [65]. Indeed, to these partitions we associate the magnetic quiver:

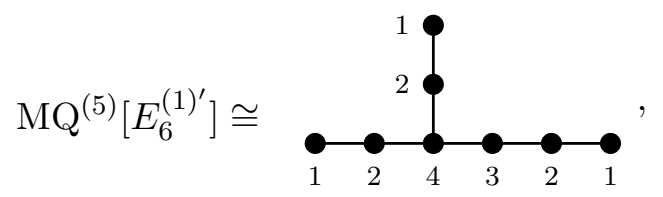

This $3 \mathrm{~d} \mathcal{N}=4$ quiver is ugly and equivalent to the $r=1 E_{6}$ quiver plus four twisted hypermultiplets, which transform in the fundamental of the $\mathrm{SU}(4)$ flavor symmetry. In the $\mathrm{MQ}^{(5)}$ (5.18), this flavor SU(4) arises as the CB symmetry associated to the right-hand leg, which is a $T[\mathrm{SU}(4)]$ quiver, and the $4 \mathrm{~d}$ twisted hypermultiplets correspond to the $\Delta=\frac{1}{2}$ monopole operators, with the flavor weights determined by their topological charges. Let us denote these hypermultiplets by $\widetilde{Q}_{i}, Q_{i}, i=1, \cdots, 4$.

The actual global symmetry of this theory $\mathcal{S}^{\prime}$ is therefore $E_{6} \times \mathrm{Sp}(4)$, with only the subgroup $\mathrm{SU}(4) \times \mathrm{SU}(2)^{2}$ manifest in the class $\mathcal{S}$ description (5.17). Crucially for our purpose, the $\mathrm{SU}(4)$ symmetry associated with the full puncture is also embedded inside $E_{6} \cdot{ }^{16}$ Consequently, its moment map takes the form

$$
\mu_{\mathrm{SU}(4)}=\left.\mu^{E_{6}}\right|_{\mathrm{SU}(4)}+\widetilde{Q}_{i} Q_{j}
$$

where $\left.\mu^{E_{6}}\right|_{\mathrm{SU}(4)}$ denotes the restriction of the $E_{6}$ moment map to the $\mathrm{SU}(4)$ subgroup. To recover the bad theory $E_{6}^{(1)}$, we need to activate a minimal nilpotent VEV for $\mu_{\mathrm{SU}(4)}$

$$
\left.\mu^{E_{6}}\right|_{\mathrm{SU}(4)}+\widetilde{Q}_{i} Q_{j}=\left(\begin{array}{cccc}
0 & 1 & 0 & 0 \\
0 & 0 & 0 & 0 \\
0 & 0 & 0 & 0 \\
0 & 0 & 0 & 0
\end{array}\right)
$$

There is an obvious ambiguity in implementing this Higgsing. Since the matrix (5.20) has rank 1, we can set $\left.\mu^{E_{6}}\right|_{\mathrm{SU}(4)}=0$, which corresponds to a VEV for the free sector only. In

\footnotetext{
${ }^{16}$ More precisely, $\mathrm{SU}(4)$ is embedded in the $\mathrm{SU}(6)$ factor of the maximal subgroup $\mathrm{SU}(6) \times \mathrm{SU}(2) \subset E_{6}$.
} 
this case, we flow to the rank- $1 E_{6}$ theory plus a free hypermultiplet. ${ }^{17}$ We then recover the description of $E_{6}^{(1)}$ argued for in section 4 .

On the other hand, if we turn on a nilpotent VEV for $\left.\mu^{E_{6}}\right|_{\mathrm{SU}(4)}$ instead, the $E_{6}$ theory is higgsed to a free theory, resulting in a collection of free hypermultiplets in the infrared. Both options are possible, but only the former option gives rise to an $r=1$ theory.

All the other $r=1$ trinions can be treated in the same way. For instance, the box-lifted version of $E_{6}^{(2)}$ reads

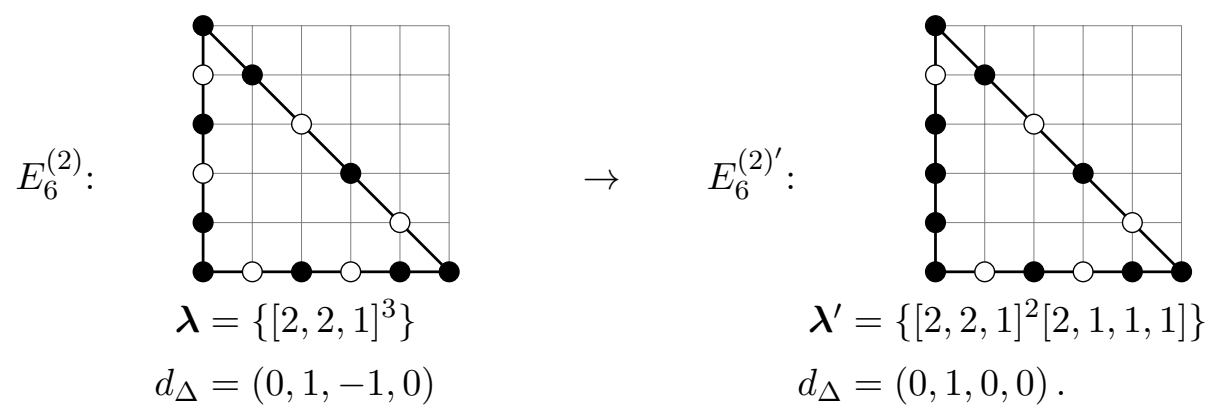

The magnetic quiver associated to the $E_{6}^{(2)^{\prime}}$ theory is again ugly. It is equivalent to the $E_{6}$ quiver plus 7 twisted hypermultiplets.

For the rank-one $E_{7}^{(k)}$ theories, we have the GTPs

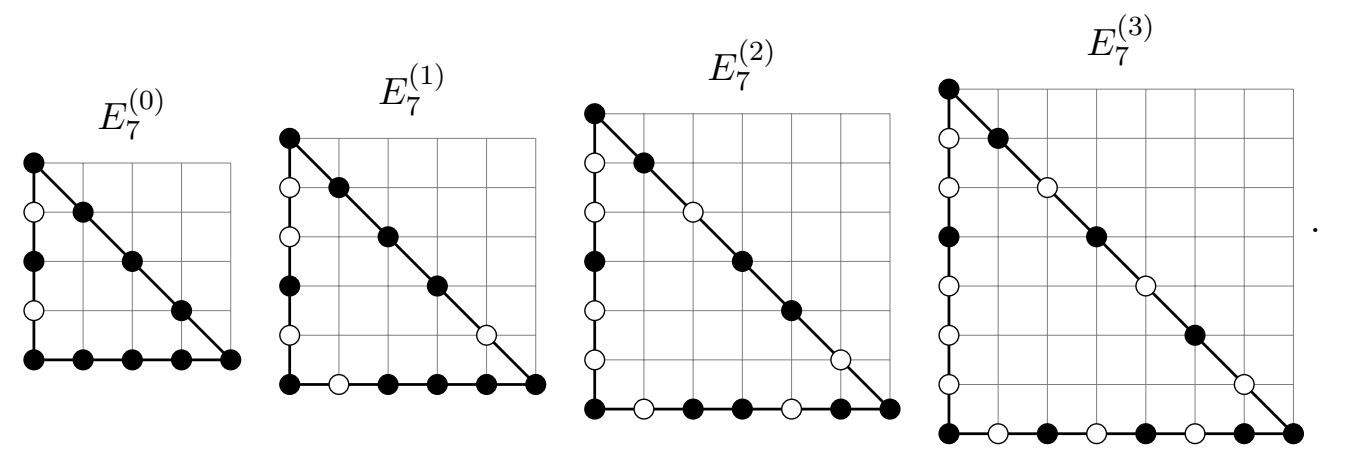

The first one is simply the rank-one $E_{7}$ theory [63]; it has a dimension vector $d_{\Delta}=(0,0,1)$. The other theories have bad magnetic quivers, and correspondingly $d_{\Delta}=(0,0,1,-1,0, \cdots)$. Note also that, for the $E_{7}^{(2)}$ GTP, the partitions along the edges are unordered, and we should reorder them before interpreting them as class $\mathcal{S}$ models (this introduces free hypermultiplet, as in the discussion around (5.6)). The box-lifting/white-dot-filling prescription gives us the following good $r=1$ models:

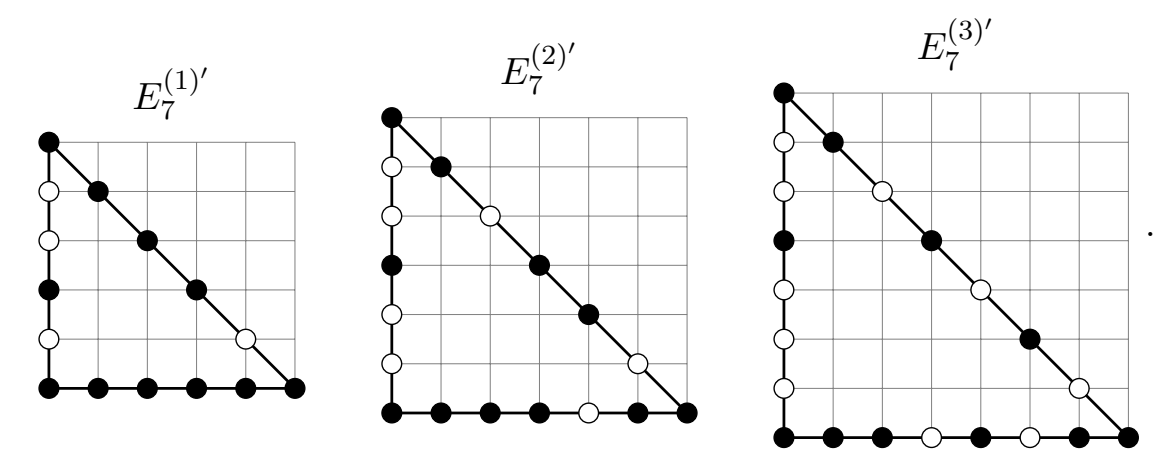

\footnotetext{
${ }^{17}$ The other three are identified with Goldstone modes for the Higgsing and we can discard them.
} 
Their magnetic quivers are all IR-equivalent to the $E_{7}$ magnetic quiver plus free twisted hypermultiplets. As always, we can focus on the $k=1$ case, which has an $\mathrm{SU}(5)$ symmetry associated to the full puncture. The partial closure of the puncture is similar to the $E_{6}^{(1)}$ case above. The $E_{8}$ series is completely similar. For $k=1$, we have:
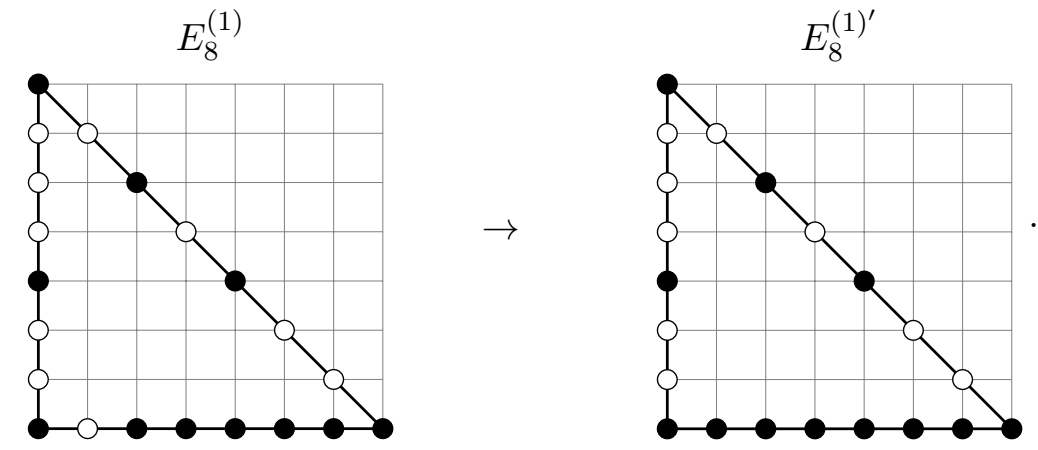

The pattern of partial closure from $E_{8}^{(1)^{\prime}}$ to $E_{8}^{(1)}$ is the same as before, with a nilpotent VEV given to free hypermultiplets charged under the full-puncture SU(7) symmetry of the $E_{8}^{(1)^{\prime}}$ model.

\subsubsection{Higher-rank theories}

Finally, let us briefly consider the general case with rank $r=\ell+1$. The important new ingredient is the presence of additional sources of badness, as in the example (5.15). Let us first review the case $k=\ell \Delta_{n}(\widehat{k}=0)$ with $\ell>0$. The partitions associated with the $E_{n}^{\left(\Delta_{n} \ell\right)}$ models are:

$$
\begin{aligned}
& \lambda_{E_{6}^{(3 \ell)}}=\left\{[\ell+1, \ell+1, \ell+1]^{3}\right\}, \\
& \lambda_{E_{7}^{(4 \ell)}}=\left\{[2 \ell+2,2 \ell+2]\left[(\ell+1)^{4}\right]^{2}\right\}, \\
& \lambda_{E_{8}^{(6 \ell)}}=\left\{[3 \ell+3,3 \ell+3][2 \ell+2,2 \ell+2,2 \ell+2]\left[(\ell+1)^{6}\right]\right\} .
\end{aligned}
$$

The 'box-lifting' prescription of [66] reads:

$$
\begin{aligned}
& \lambda_{E_{6}^{(3 \ell)^{\prime}}}=\left\{[\ell+1, \ell+1, \ell+1]^{2}[\ell+1, \ell+1, \ell, 1]\right\}, \\
& \lambda_{E_{7}^{(4 \ell)^{\prime}}}^{\prime}=\left\{[2 \ell+2,2 \ell+2]\left[(\ell+1)^{4}\right]\left[(\ell+1)^{3}, \ell, 1\right]\right\}, \\
& \lambda_{E_{8}^{\prime(6 \ell)^{\prime}}}^{\prime}=\left\{[3 \ell+3,3 \ell+3][2 \ell+2,2 \ell+2,2 \ell+2]\left[(\ell+1)^{5}, \ell, 1\right]\right\} .
\end{aligned}
$$

For instance, for the rank- $2 E_{6}$ theory, we have:




The magnetic quivers associated to (5.25) are given by $\ell+1$ times the affine $E_{n}$ quiver (that is, the ranks are given by $(\ell+1) d_{i}$, with $d_{i}$ the Dynkin labels) while the magnetic quivers associated to (5.26) are given by:

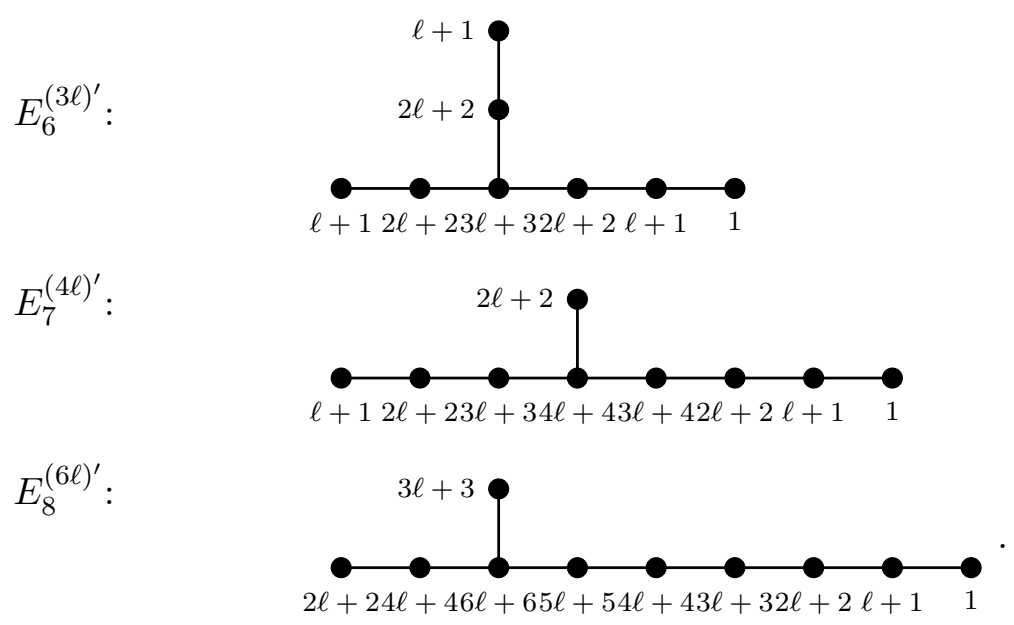

These magnetic quivers are 'good', due to the additional U(1) factor at the end of the left-hand tail. The corresponding Higgs-branch flavor symmetry of the class $\mathcal{S}$ theory is $E_{n} \times \mathrm{SU}(2)$. The $\mathrm{CB}$ of the magnetic quivers (5.28) reproduce the correct moduli space of $(\ell+1) E_{n}$ instantons, as studied e.g. in [44, 103].

The uplifting procedure for $\widehat{k}>0$ is similar. It gives rise to the same magnetic quivers (5.28) plus free twisted hypermultiplets. For completeness, we list the partitions after the box-lifting in tables 7, 8 and 9 . There we listed the unordered partitions, corresponding to GTP, which are equivalent to ordered partitions plus free hypermultiplets.

\section{Classification of the $\mathrm{SU}(N)$ trinions}

In the previous sections, we focussed on 'trinion' singularities of type I, in the notation of (2.57). The corresponding $4 \mathrm{~d}$ theory $\mathscr{T}_{\mathbf{X}}^{4 \mathrm{~d}}$ is a $D_{p}^{b}(\mathrm{SU}(N))$-trinions with $b=N$ for the three legs. In this section, we give a complete classification of the $D_{p}^{b}(\mathrm{SU}(N))$ trinions

$$
D_{p_{1}}^{b_{1}}(\mathrm{SU}(N))-\underbrace{D_{p_{2}}^{b_{2}}(\mathrm{SU}(N))}_{N} D_{p_{3}}^{b_{3}}(\mathrm{SU}(N)) .
$$

As discussed in section 2, the vanishing of the $\mathrm{SU}(N) \beta$-function is equivalent to the condition

$$
\sum_{\alpha=1}^{3} \frac{b_{\alpha}}{p_{\alpha}}=N .
$$

Given a solution, the $\mathcal{N}=2 \mathrm{SCFT}$ is realized by the isolated hypersurface singularity (IHS)

$$
x^{N}+x^{N-b_{1}} z_{1}^{p_{1}}+x^{N-b_{2}} z_{2}^{p_{2}}+x^{N-b_{3}} z_{3}^{p_{3}}+\cdots=0 .
$$




\begin{tabular}{|c|c|c|c|c|c|c|c|}
\hline Type & $(a, b, c, d)$ & $\mathrm{AD}\left[G, G^{\prime}\right]$ & $r$ & $f$ & $d_{\mathrm{H}}$ & $\widehat{r}$ & $\widehat{d_{\mathrm{H}}}$ \\
\hline $\mathrm{II}$ & $\{2,2 N, N, 2\}$ & {$\left[A_{2 N-1}, D_{N+1}\right]$} & 0 & $N+1$ & $N(N+1)$ & $N^{2}-1$ & $N+1$ \\
\hline $\mathrm{VIII}_{2}$ & $\{2 k-1,2 k-1,2,2\}$ & {$\left[D_{2 k}, D_{2 k}\right]$} & $k-1$ & $2 k+2$ & $2 k^{2}+k+1$ & $(2 k+1)(k-1)$ & $3 k+1$ \\
\hline $\mathrm{VIII}_{2}$ & $\{2 k, 2 k, 2,2\}$ & {$\left[D_{2 k+1}, D_{2 k+1}\right]$} & $k-1$ & $2 k+1$ & $(k+1)(2 k+1)$ & $k(2 k+1)$ & $3 k$ \\
\hline
\end{tabular}

Table 10. Basic data for the infinite series of $\mathrm{SU}(N)$ trinions. Here $(a, b, c, d)$ indicate the powers in the hypersurface equation. For the $\left[D_{N+1}, D_{N+1}\right]$ series, which are given by type $\operatorname{VIII}_{2}(N, N, 2,2)$ singularities, or equivalently by type IV $(N, 2, N, 2)$ singularities, we look at odd and even $N$ separately. They all have $\Delta \mathcal{A}_{r}=0, b_{3}=0$ and trivial one-form symmetry.

There are four subfamilies of solutions, depending on the choices for $b_{\alpha} \in\{N, N-1\}$. In terms of the Yau-Yu classification [85], we have

$$
\begin{array}{lll}
\left(b_{\alpha}\right)=(N, N, N) & : & \mathrm{I}\left(p_{1}, p_{2}, p_{3}, N\right), \\
\left(b_{\alpha}\right)=(N, N, N-1) & : & \mathrm{II}\left(p_{1}, p_{2}, N, p_{3}\right), \\
\left(b_{\alpha}\right)=(N, N-1, N-1) & : & \operatorname{VIII}\left(p_{1}, N, p_{2}, p_{3}\right), \\
\left(b_{\alpha}\right)=(N-1, N-1, N-1) & : & \operatorname{XIV}\left(N, p_{1}, p_{2}, p_{3}\right) .
\end{array}
$$

Note that, for the cases of type VIII and XIV, the singularity (6.3) contains additional monomials so that the singularity is isolated $[54,55]$. This introduces some subtypes in the YY classification, which will be discussed in more detail elsewhere [37].

We already discussed at length the type I solutions, which are the $E_{n}^{(k)}$ models. The other solutions to (6.2) are easily classified, and the corresponding theories will be described below. They are summarized in table 10 and in tables 11, 12, 13 and 14, where we display the numbers $r, f, d_{\mathrm{H}}$, which characterize the $5 \mathrm{~d}$ theory $\mathcal{T}_{\mathbf{X}}^{5 \mathrm{~d}}$, as well as the electric quivers $\mathrm{EQ}^{(4)}$ for the $D_{p}^{b}(\mathrm{SU}(N))$ trinions. We have also indicated in the tables whether the resolved Calabi-Yau threefold is smooth, and whether any of the divisors of the resolution are singular. The details of the crepant resolutions are presented in appendix A. From these results, we observe that $\mathscr{T}_{\mathbf{X}}^{4 \mathrm{~d}}$ is a Lagrangian $4 \mathrm{~d} \mathcal{N}=2$ SCFT (a conformal special unitary quiver) if the resolved geometry is smooth, and the divisors have at most codimensionone singularities on them (no point singularity). On the other hand, if there are residual terminal singularities after the resolution, the $4 \mathrm{~d}$ theory $\mathscr{T}_{\mathbf{X}}^{4 \mathrm{~d}}$ is non-Lagrangian and the $\mathrm{EQ}^{(4)}$ is a mixed $\mathrm{SU}(n)-\mathrm{U}(m)$ quiver.

\subsection{Case $\left(b_{\alpha}\right)=(N, N, N)$}

In this case, the $N$ dependence drops out from (6.2), and we therefore obtain infinite families of solutions indexed by $N$ :

$$
\sum_{\alpha=1}^{3} \frac{1}{p_{\alpha}}=1 \quad \Rightarrow \quad\left(p_{\alpha}\right)=(3,3,3),(2,4,4),(2,3,6) .
$$


When $\left(p_{\alpha}\right)=(3,3,3)$, the theory corresponds to the $E_{6}^{(N-3)}$ theory. When $\left(p_{\alpha}\right)=(2,4,4)$, the theory corresponds to the $E_{7}^{(N-4)}$. When $\left(p_{\alpha}\right)=(2,3,6)$, the theory corresponds to the $E_{8}^{(N-6)}$ theory. These models were already discussed at length in sections 3 and 4.

\subsection{Case $\left(b_{\alpha}\right)=(N, N, N-1)$}

Consider the condition

$$
\frac{N}{p_{1}}+\frac{N}{p_{2}}+\frac{N-1}{p_{3}}=N
$$

This has the following obvious solutions for any $N$ :

$$
\left(p_{\alpha}\right)=(2,2 N, 2), \quad \forall N
$$

They are terminal singularities of Type $\operatorname{II}(2,2 N, N, 2)$. These singularities engineer the generalized Argyres-Douglas theory $\mathrm{AD}\left[A_{2 N-1}, D_{N+1}\right]$ in Type IIB [67]. After the dimensional reduction of $D_{p}^{b}(\mathrm{SU}(N))$ to $3 \mathrm{~d}$, we therefore obtain an explicit 'electric quiver description' for these $4 \mathrm{~d}$ SCFTs (for $k>1$ )

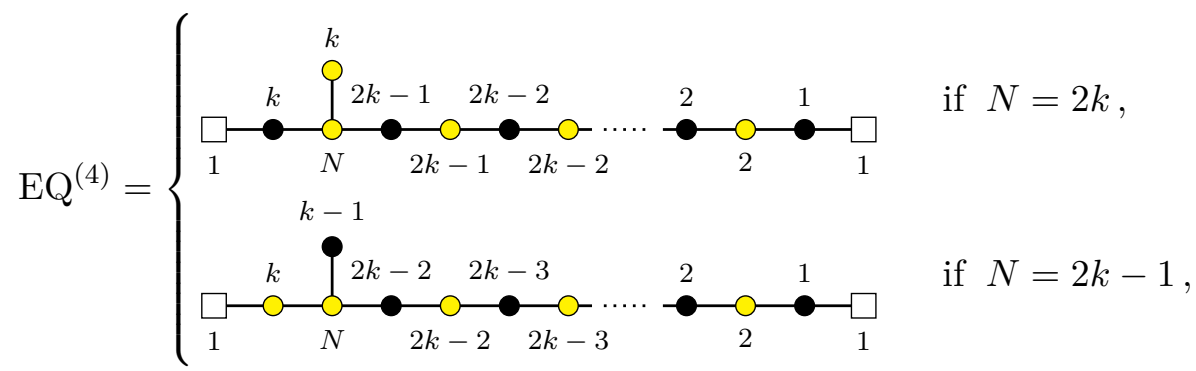

Since these singularities have $r=0$, the 5 d theory consists of $d_{\mathrm{H}}=N(N+1)$ free hypermultiplets. To see this explicitly, we can obtain the $\mathrm{MQ}^{(5)}$ from (6.8), corresponding to replacing all the $\mathrm{SU}(n)$ nodes by $\mathrm{U}(n)$ nodes, including the central one, because there is no higher-form symmetry associated to the singularity $(\mathfrak{f}=0)$. The resulting unitary quiver is ugly, and repeated applications of the GW duality gives us exactly $d_{\mathrm{H}}$ twisted hypermultiplets.

In addition, there are three sporadic solutions to (6.6)

\begin{tabular}{|c|c|ccc|}
\hline Sporadic Models (\#) & $N$ & $p_{1}$ & $p_{2}$ & $p_{3}$ \\
\hline$(1)$ & 6 & 3 & 4 & 2 \\
$(2)$ & 10 & 2 & 5 & 3 \\
$(3)$ & 15 & 3 & 5 & 2 \\
\hline
\end{tabular}

The basic properties and the $\mathrm{EQ}^{(4)}$ for these models are shown in table 11. In appendix B, we prove that this set of solutions is complete.

For the model (1), from the resolution of the geometry in appendix A.2, we see that this is a rank- $1 E_{6}$ theory (trivially) coupled to the rank-0 theory of type $E_{8}^{(-3)}$, which is indicated by the fact that there is a remnant terminal singularity. This again means that the 'naive' $\mathrm{MQ}^{(5)}$ is ugly. Since there is no one-form symmetry, we replace all the $\mathrm{SU}(N)$ 


\begin{tabular}{|c|c|c|c|c|c|c|}
\hline$(\#)$ & $\begin{array}{l}\text { Type }(a, b, c, d) \\
F\left(x_{1}, x_{2}, x_{3}, x_{4}\right)\end{array}$ & $r$ & $f$ & $d_{\mathrm{H}}$ & Smooth CY/Divs & $\mathrm{EQ}^{(4)}$ \\
\hline (1) & $\begin{array}{c}\mathrm{II}_{1}(3,4,6,2) \\
x_{1}^{3}+x_{2}^{4}+x_{3}^{6}+x_{4}^{2} x_{3}\end{array}$ & 1 & 4 & 23 & (Singular, Singular) & $\underset{2}{c}$ \\
\hline (2) & $\begin{array}{c}\mathrm{II}_{1}(2,5,10,3) \\
x_{1}^{2}+x_{2}^{5}+x_{3}^{10}+x_{4}^{3} x_{3}\end{array}$ & 2 & 8 & 46 & (Smooth, Singular) & $\mathrm{O}_{2}$ \\
\hline (3) & $\begin{array}{c}\mathrm{II}_{1}(3,5,15,2) \\
x_{1}^{3}+x_{2}^{5}+x_{3}^{15}+x_{4}^{2} x_{3}\end{array}$ & 4 & 8 & 68 & (Smooth, Singular) & $\mathrm{3}_{3}$ \\
\hline
\end{tabular}

Table 11. Basic data and $\mathrm{EQ}^{(4)}$ for the first three 'sporadic' $\mathrm{SU}(N)$ trinions. They all have $\Delta \mathcal{A}_{r}=$ $0, b_{3}=0$ and trivial one-form symmetry. The $\mathrm{EQ}^{(4)}$ that only have $\mathrm{SU}(N)$ gauge groups (yellow nodes) correspond to Lagrangian $\mathcal{N}=2$ SCFTs in $4 \mathrm{~d}$. The (smooth/singular, smooth/singular) indicates whether the Calabi-Yau threefold is singular and there are still singular divisors in the resolution, respectively.

by $\mathrm{U}(N)$ nodes in $\mathrm{EQ}^{(4)}$. We can then check that the $\mathrm{MQ}^{(5)}$ is equivalent to the $E_{6}$ quiver plus 12 twisted hypermultiplets

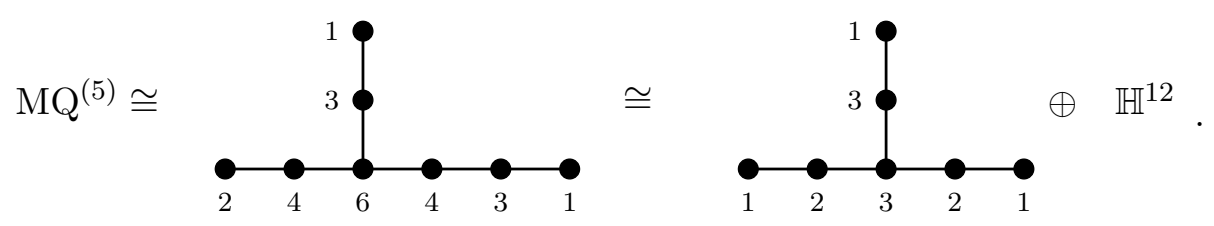

For the sporadic models (2) and (3), there is no residual terminal singularity, but there are singular divisors. The singularities are codimension one on the divisors. The model (2) has $r=2$ and was studied in [36]. Its Higgs branch, as determined from the $\mathrm{MQ}^{(5)}$, is the next-to-minimal nilpotent orbit of $\mathfrak{e}_{8}$ [104]. The $\mathrm{MQ}^{(5)}$ of model (3) appeared before in $[105]$.

In addition, we also have the solutions $N=2,\left(p_{\alpha}\right)=(2,3,3), N_{3}, N=3,\left(p_{\alpha}\right)=$ $(3,3,2), N=4,\left(p_{\alpha}\right)=(2,4,3)$ and $N=6,\left(p_{\alpha}\right)=(2,3,5)$, but they are equivalent to the type-I singularities $(2,2,3,6),(3,3,3,3),(2,4,4,4)$ and $(2,3,6,6)$, respectively. The first one is the rank-0 theory $E_{8}^{(-4)}$, and the other theories are rank-1 $E_{6}, E_{7}$ and $E_{8}$ theories respectively.

\subsection{Case $\left(b_{\alpha}\right)=(N, N-1, N-1)$}

Next, consider the condition

$$
\frac{N}{p_{1}}+\frac{N-1}{p_{2}}+\frac{N-1}{p_{3}}=N
$$




\begin{tabular}{|c|c|c|c|c|c|c|}
\hline$(\#)$ & $\begin{array}{l}\text { Type }(a, b, c, d) \\
F\left(x_{1}, x_{2}, x_{3}, x_{4}\right)\end{array}$ & $r$ & $f$ & $d_{\mathrm{H}}$ & Smooth CY/Divs & $\mathrm{EQ}^{(4)}$ \\
\hline (4) & $\begin{array}{c}\mathrm{VIII}_{2}(3,5,2,3) \\
x_{1}^{3}+x_{2}^{5}+x_{3}^{2} x_{2}+x_{4}^{3} x_{2} \\
+x_{1} x_{3} x_{4}\end{array}$ & 1 & 3 & 18 & (Singular, Singular) & $\underset{1}{\bullet} \bullet_{3}$ \\
\hline (5) & $\begin{array}{c}\mathrm{VIII}_{1}(2,7,3,4) \\
x_{1}^{2}+x_{2}^{7}+x_{3}^{3} x_{2}+x_{4}^{4} x_{2} \\
+x_{3}^{2} x_{4}^{2}\end{array}$ & 1 & 5 & 30 & (Singular, Singular) & $\longrightarrow$ \\
\hline (6) & $\begin{array}{c}\mathrm{VIII}_{2}(3,9,2,4) \\
x_{1}^{3}+x_{2}^{9}+x_{3}^{2} x_{2}+x_{4}^{4} x_{2} \\
+x_{1} x_{4}^{3}\end{array}$ & 3 & 8 & 39 & (Smooth, Smooth) & 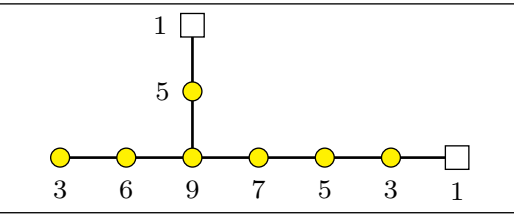 \\
\hline (7) & $\begin{array}{c}\mathrm{VIII}_{2}(4,10,2,3) \\
x_{1}^{4}+x_{2}^{10}+x_{3}^{2} x_{2}+x_{4}^{3} x_{2} \\
+x_{1} x_{3} x_{4}\end{array}$ & 3 & 5 & 41 & (Singular, Singular) & $\overbrace{2}^{-} \underbrace{}_{5}$ \\
\hline
\end{tabular}

Table 12. Basic data and $\mathrm{EQ}^{(4)}$ for the 'sporadic' $\mathrm{SU}(N)$ trinions, continued $(2 / 4)$.

\begin{tabular}{|c|c|c|c|c|c|c|}
\hline$(\#)$ & $\begin{array}{l}\text { Type }(a, b, c, d) \\
F\left(x_{1}, x_{2}, x_{3}, x_{4}\right)\end{array}$ & $r$ & $f$ & $d_{\mathrm{H}}$ & Smooth CY/Divs & $\mathrm{EQ}^{(4)}$ \\
\hline (8) & $\begin{array}{c}\mathrm{VIII}_{1}(2,16,3,5) \\
x_{1}^{2}+x_{2}^{16}+x_{3}^{3} x_{2}+x_{4}^{5} x_{2} \\
+x_{3}^{2} x_{4}^{2}\end{array}$ & 4 & 9 & 76 & (Smooth, Singular) & $\underset{1}{\square-0}$ \\
\hline (9) & $\begin{array}{c}\mathrm{VIII}_{2}(3,21,2,5) \\
x_{1}^{3}+x_{2}^{21}+x_{3}^{2} x_{2}+x_{4}^{5} x_{2} \\
+x_{1} x_{3} x_{4}\end{array}$ & 7 & 9 & 98 & (Smooth, Singular) & $\underbrace{11}_{7}$ \\
\hline (10) & $\begin{array}{c}\mathrm{VIII}_{2}(5,25,2,3) \\
x_{1}^{5}+x_{2}^{25}+x_{3}^{2} x_{2}+x_{4}^{3} x_{2} \\
+x_{1} x_{3} x_{4}\end{array}$ & 10 & 9 & 115 & (Smooth, Singular) & $\bigcirc_{5}^{10} \sim_{15}$ \\
\hline
\end{tabular}

Table 13. Basic data and $\mathrm{EQ}^{(4)}$ for the 'sporadic' $\mathrm{SU}(N)$ trinions, continued (3/4). 


\begin{tabular}{|c|c|c|c|c|c|c|}
\hline$(\#)$ & $\begin{array}{l}\text { Type }(a, b, c, d) \\
F\left(x_{1}, x_{2}, x_{3}, x_{4}\right)\end{array}$ & $r$ & $f$ & $d_{\mathrm{H}}$ & Smooth CY/Divs & $\mathrm{EQ}^{(4)}$ \\
\hline (11) & $\begin{array}{c}\mathrm{XIV}_{3}(7,2,3,3) \\
x_{1}^{7}+x_{2}^{2} x_{1}+x_{3}^{3} x_{1}+x_{4}^{3} x_{1} \\
+x_{2} x_{3}^{2}+x_{2} x_{4}^{2}+x_{2} x_{3} x_{4}\end{array}$ & 3 & 8 & 29 & (Smooth, Smooth) & $\square_{1}^{\square} \underbrace{}_{3}$ \\
\hline$(12)$ & $\begin{array}{c}\mathrm{XIV}_{4}(13,2,3,4) \\
x_{1}^{13}+x_{2}^{2} x_{1}+x_{3}^{3} x_{1}+x_{4}^{4} x_{1} \\
+x_{3} x_{4}^{3}+2 x_{2} x_{3} x_{4}\end{array}$ & 6 & 9 & 57 & (Smooth, Smooth) & $\underbrace{}_{1}$ \\
\hline (13) & $\begin{array}{c}\mathrm{XIV}_{4}(31,2,3,5) \\
x_{1}^{31}+x_{2}^{2} x_{1}+x_{3}^{3} x_{1}+x_{4}^{5} x_{1} \\
+3 x_{2} x_{3} x_{4}\end{array}$ & 15 & 10 & 145 & (Smooth, Singular) & $\overbrace{1}^{\square}$ \\
\hline
\end{tabular}

Table 14. Basic data and $\mathrm{EQ}^{(4)}$ for the 'sporadic' $\mathrm{SU}(N)$ trinions, continued $(4 / 4)$.

There are two infinite series of solutions

$$
\left(p_{1}, p_{2}, p_{3}\right)=(N, 2,2),(2,2,2 N-2), \forall N .
$$

For the first series, one needs to add marginal deformation terms in (6.3) to obtain the following IHS of type $\operatorname{VIII}_{2}(N, N, 2,2)$

$$
x_{1}^{N}+x_{2}^{N}+x_{1} x_{3}^{2}+x_{1} x_{4}^{2}+x_{2} x_{3} x_{4}=0 .
$$

In fact, this isolated singularity is isomorphic to a singularity of type $\operatorname{IV}(N, 2, N, 2)$

$$
x_{1}^{N}+x_{1} x_{2}^{2}+x_{3}^{N}+x_{3} x_{4}^{2}=0,
$$

which gives rise to $\mathrm{AD}\left[D_{N+1}, D_{N+1}\right]$ theories $[67,74]$. We list its geometric data in table 10 . For $N=2 k-1$, the $5 \mathrm{~d}$ SCFT is the UV fixed point of the $5 \mathrm{~d} \mathcal{N}=1$ gauge theory

$$
\mathrm{SU}(k)_{ \pm \frac{3}{2}}+(2 k+1) \boldsymbol{F},
$$

which has flavor symmetry algebra is $\mathfrak{g}_{F}=\mathfrak{s o}(4 k+2) \oplus \mathfrak{u}(1)$. For $N=2 k$, the theory is the same SCFT coupled to $2 k$ free hypermultiplets, which correspond to $2 k$ conifold singularities in the crepant resolution. The $\mathrm{EQ}^{(4)}$ for these infinite sequences are

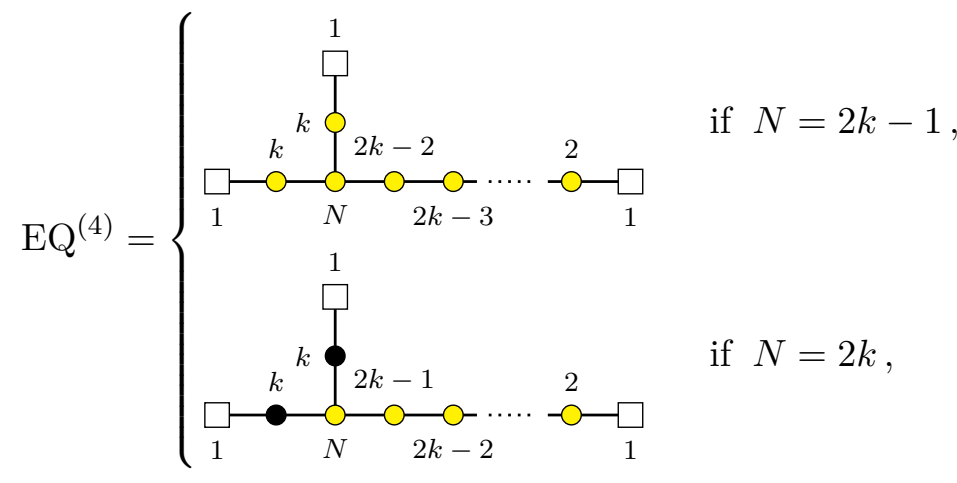


The $\mathrm{MQ}^{(5)}$ is obtained by replacing all the $\mathrm{SU}(n)$ nodes in $\mathrm{EQ}^{(4)}$ by $\mathrm{U}(n)$ nodes. The quiver for $N=2 k$ is ugly, and can be dualized to extract the free sector, so that we obtain

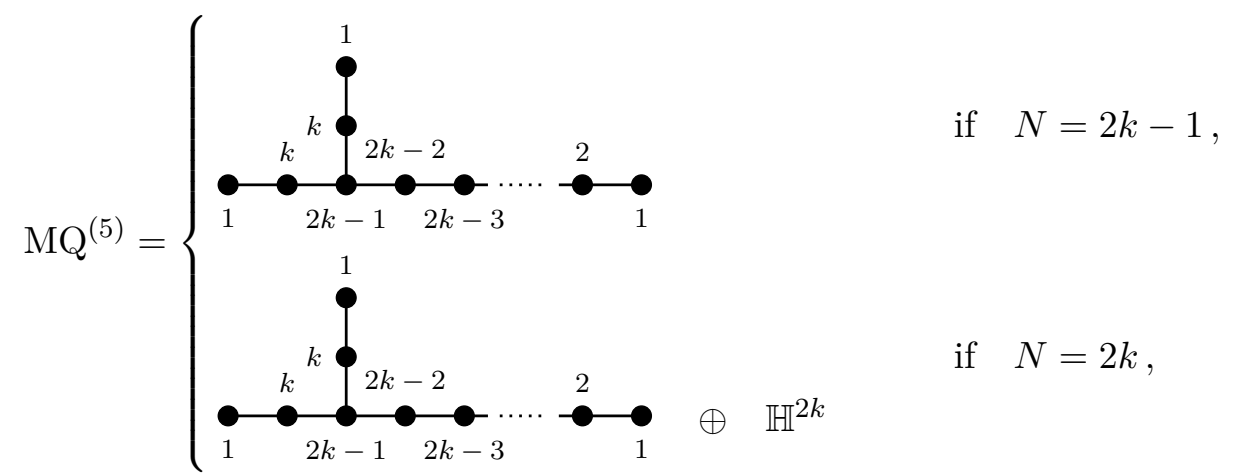

One can check that, for $N=2 k-1$, the magnetic quiver is indeed the ones of $\mathrm{SU}(k)_{ \pm \frac{3}{2}}+$ $(2 k+1) \boldsymbol{F}[46]$. This gives a nice consistency check on our geometric approach. The second series in (6.12) is equivalent to (6.7), i.e. we have the equivalences

$$
\operatorname{VIII}(2,2, N, 2 N-2) \cong \mathrm{II}(2,2 N, N, 2) \text {. }
$$

In addition, we find the sporadic solutions

\begin{tabular}{|c|c|ccc|}
\hline$(\#)$ & $N$ & $p_{1}$ & $p_{2}$ & $p_{3}$ \\
\hline$(4)$ & 5 & 3 & 2 & 3 \\
$(5)$ & 7 & 2 & 3 & 4 \\
$(6)$ & 9 & 3 & 2 & 4 \\
$(7)$ & 10 & 4 & 2 & 3 \\
$(8)$ & 16 & 2 & 3 & 5 \\
$(9)$ & 21 & 3 & 2 & 5 \\
$(10)$ & 25 & 5 & 2 & 3 \\
\hline
\end{tabular}

Here, we omitted the solution $N=4,\left(p_{\alpha}\right)=(2,3,3)$ because it is equivalent to the model $\mathrm{I}(2,4,4,4)$, the rank- $1 E_{7}$ theory.

For the model (4), we deduce from the resolution geometry that it is a rank- $1 E_{6}$ theory coupled to a rank-0 theory $E_{8}^{(-4)}$ plus another conifold (a free hypermultiplet). From $\mathrm{EQ}^{(4)}$, after gauging and rebalancing ugly nodes, we indeed get

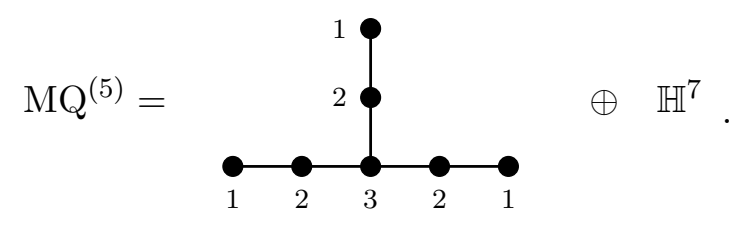

Similarly, the model (5) is a rank- $1 E_{7}$ theory coupled to a rank-0 theory $E_{8}^{(-3)}$ and another conifold. From gauging and rebalancing $\mathrm{EQ}^{(4)}$, we get

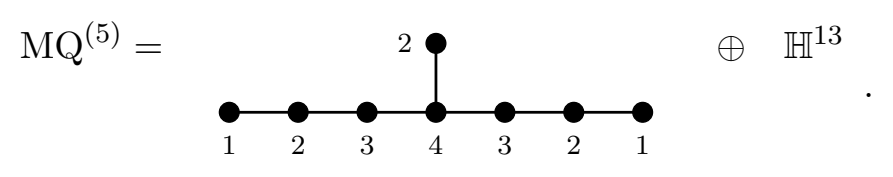


The model (6) has a smooth resolution, and correspondingly $\mathscr{T}_{\mathbf{X}}^{4 \mathrm{~d}}$ is a Lagrangian theory, with the $\mathrm{SU}(n)$ quiver shown in table 13. The corresponding $5 \mathrm{~d} \operatorname{SCFT} \mathcal{T}_{\mathbf{X}}^{5 \mathrm{~d}}$ has $r=3$ and $d_{\mathrm{H}}=39$. The structure of its Higgs branch can be studied using the magnetic quiver

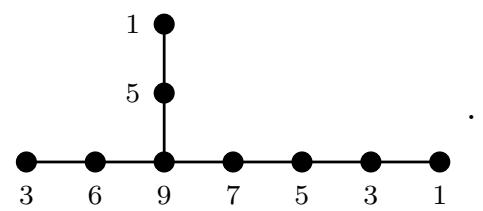

In particular, the 5 d flavor symmetry is $\mathfrak{g}_{F}=\mathfrak{e}_{7} \oplus \mathfrak{u}(1)$.

The model (7) has a crepant resolutions with a residual terminal singularity $E_{8}^{(-3)}$. After gauging the $\mathrm{U}(1)^{f}$ of $\mathrm{EQ}^{(4)}$ and rebalancing, we get

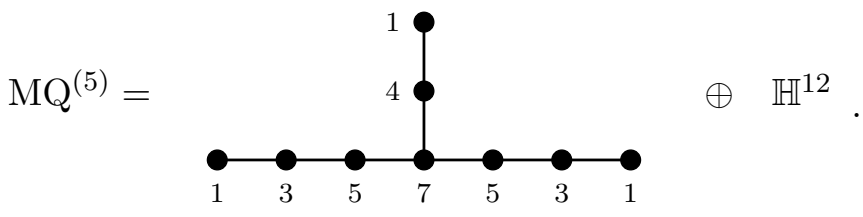

Note that the magnetic quiver of the interacting sector is exactly the same as the model (11) in table 14, which corresponds to a $r=3, f=85 \mathrm{~d}$ SCFT. Thus, we conclude that the $5 \mathrm{~d}$ SCFT for model (7) is equivalent to the one of model (11) plus 12 hypermultiplets.

The model (8), (9) and (10) all have smooth crepant resolutions, and correspondingly $\mathscr{T}_{\mathbf{X}}^{4 \mathrm{~d}}$ is a Lagrangian theory. From the magnetic quivers, we can show that they each have 5 d global symmetry $\mathfrak{g}_{F}=\mathfrak{e}_{8} \oplus \mathfrak{s u}(2)$.

\subsection{Case $\left(b_{\alpha}\right)=(N-1, N-1, N-1)$}

Lastly, consider the condition

$$
\frac{N-1}{p_{1}}+\frac{N-1}{p_{2}}+\frac{N-1}{p_{3}}=N .
$$

For any $N$, we have the solution $\left(p_{\alpha}\right)=(2,2, N-1)$, but it turns out this is equivalent to the second series in (6.12), namely

$$
\mathrm{XIV}_{2}(N, 2,2, N-1) \cong \operatorname{VIII}_{2}(N, N, 2,2) \cong \operatorname{IV}(N, 2, N, 2) .
$$

We also find the sporadic solutions

\begin{tabular}{|c|c|ccc|}
\hline$(\#)$ & $N$ & $p_{1}$ & $p_{2}$ & $p_{3}$ \\
\hline$(11)$ & 7 & 2 & 3 & 3 \\
$(12)$ & 13 & 2 & 3 & 4 \\
$(13)$ & 31 & 2 & 3 & 5 \\
\hline
\end{tabular}

These three models have smooth resolutions and $\mathscr{T}_{\mathbf{X}}^{4 \mathrm{~d}}$ is Lagrangian. The 5d SCFT for model (11) has $r=3$ and $\mathfrak{g}_{F}=\mathfrak{e}_{6} \oplus \mathfrak{s u}(2)$. The model (12) has rank $r=6$ and $\mathfrak{g}_{F}=\mathfrak{e}_{7} \oplus \mathfrak{s u}(2)$. Finally, the model (13) has $r=15$ and $\mathfrak{g}_{F} \supset \mathfrak{e}_{8}$. In appendix B, we prove that there are no more solutions to the equations (6.6), (6.11) and (6.24). This completes our classification. 


\section{$7 \quad D_{p}^{b}(G)$-trinions SCFTs for $G=D, E$}

In this section, we generalize the four-dimensional trinion SCFTs to the cases with a central gauge group of type $D$ or $E$. We focus on the $D_{p}^{b}(G)$ trinions that can be engineered by threefold singularities in Type IIB. This leads us to discover a number of new S-dualities between SCFTs with different gauge groups, which we discuss in section 8 .

\subsection{Trinion geometries for generic $G$}

The analysis of section 2.4 can be generalized to the case of gaugings of three $D_{p}^{b}(G)$ theories with $G$ of special orthogonal or exceptional type. In the general case, however, we just have a Landau-Ginzburg description instead of a threefold singularity [56, 67]. The LG superpotential reads

$$
\mathcal{W}=w^{2}+f(t, x)+\sum_{\alpha=1}^{3} z_{\alpha}^{p_{\alpha}} f_{b_{\alpha}}(x, t),
$$

where $W_{G}(w, x, t) \equiv w^{2}+f(t, x)=0$ is the ADE singularity of type $G$ and $f_{b_{\alpha}}(x, t) z_{\alpha}^{p_{\alpha}}$ denotes the $z$-dependent part of the threefold singularity describing $D_{p_{\alpha}}^{b_{\alpha}}(G)$, shown in table 1. From a LG model point of view, $w$ in (7.1) is a massive field and can be integrated out, leaving five fields. The conformality condition reads

$$
\frac{b_{1}}{p_{1}}+\frac{b_{2}}{p_{2}}+\frac{b_{3}}{p_{3}}=h^{\vee}(G) .
$$

Importantly, if the singularity $\mathcal{W}=0$ is non-isolated, one needs to add marginal terms to (7.1) in order to have an isolated singularity at the origin, so that the LG model is well-defined.

We will consider the special case when the LG model is equivalent to a model with at most four fields. This happens whenever at least one of the fields $z_{\alpha}$ is massive. For $p=2$ or $p=1$ and an appropriate choice of $b$, we have:

$$
\mathcal{W} \supset z^{p} f_{b}(x, t)=z^{2} \quad \text { or } \quad \mathcal{W} \supset z^{p} f_{b}(x, t)=t z, x z .
$$

Such massive fields can be integrated out. If we choose $p=2$ for one of the legs, we are therefore considering a theory $\mathscr{T}_{\mathbf{X}}^{4 \mathrm{~d}}$ of the form

$$
\begin{gathered}
D_{2}^{h^{\vee}}(G) \\
D_{p_{2}}^{b_{2}}(G)-G-D_{p_{3}}^{b_{3}}(G) .
\end{gathered}
$$

The constraint (7.2) now implies that the other two $D_{p}^{b}(G)$ sectors should satisfy the relation

$$
\frac{b_{2}}{p_{2}}+\frac{b_{3}}{p_{3}}=\frac{h^{\vee}(G)}{2} \text {. }
$$

Since we are now left with only four fields, the system can be understood as a threefold compactification in Type IIB. The second option is $p=1$, which gives a mass term (7.3) 
for $b \neq h^{\vee}(G)$, since in that case the $f_{b}(x, t)$ function appearing in table 1 is always linear in either $x$ or $t$, we can simply choose the $D_{p_{1}}^{b_{1}}(G)$ theory to be $D_{1}^{b}(G)$ with $b \neq h^{\vee}(G)$ (we remind the reader that $D_{1}^{b}(G)$ is trivial for $b=h^{\vee}(G)$ ) and, due to (7.2), we take the other two $D_{p}^{b}(G)$ models to satisy the constraint

$$
\frac{b_{2}}{p_{2}}+\frac{b_{3}}{p_{3}}=h^{\vee}(G)-b
$$

Under these conditions, we can integrate out both $z_{1}$ and either $x$ or $t$ (the variable appearing in $\left.f_{b_{1}}(x, t)\right)$. We can now simply introduce a new massive field $y$ which enters quadratically in the superpotential. The threefold singularity is now given by a hypersurface in the four variables $z_{2}, z_{3}, y$, and $x$ or $t$ (the variable we have not integrated out). We can therefore also consider the family of trinions

$$
\begin{gathered}
D_{1}^{b}(G) \\
D_{p_{2}}^{b_{2}}(G)- \\
\mid
\end{gathered}-D_{p_{3}}^{b_{3}}(G)
$$

satisfying the condition (7.6). In the following, we list all such models by solving equations (7.5) and (7.6) and integrating out the massive LG fields. We collect the resulting theefold singularities and their basic properties in various tables in appendix C.

\section{2 $D_{p}^{b}(G)$-trinions with $b_{1}=h^{\vee}$ and $p_{1}=2$}

7.2.1 $b_{2}=b_{3}=h^{\vee}$

If we choose $b_{1}=b_{2}=h^{\vee}$, we have the universal solutions:

$$
\left(p_{2}, p_{3}\right)=(4,4),(3,6),
$$

for any $G$. They are the only solutions, in this case. For $G=\mathrm{SU}(N)$, these are the $E_{7}^{(N-4)}$ and $E_{8}^{(N-6)}$ series. For $\mathrm{SO}(2 N)$, the IHS equation reads

$$
x^{N-1}+x t^{2}+z_{2}^{p_{2}}+z_{3}^{p_{3}}=0
$$

The basic properties of these models, for $G=\mathrm{SO}(2 N)$ and $G=E_{n}$, are listed in table 15 .

\subsubsection{Other sporadic models}

For $G=\mathrm{SO}(2 N)$ (so that $h^{\vee}=2 N-2$ ), we also have the sporadic models in table 16. For $G=E_{6}$, we have $h^{\vee}=12$ and the allowed values of $b$ are $12,9,8$. The sporadic models are in table 17. For $G=E_{7}$, we have $h^{\vee}=18$ and the allowed values of $b$ are 18 and 14 . We then find the sporadic models in table 18. For $G=E_{8}$, we have $h^{\vee}=30$ and the allowed values of $b$ are 30,24,20, with the sporadic models in table 19 . 


\section{3 $D_{p}^{b}(G)$-trinions with $b_{1} \neq h^{\vee}$ and $p_{1}=1$}

Let us also consider models with $b_{1} \neq h^{\vee}$ and $p_{1}=1$. For $G=\operatorname{SO}(2 N)$, we have to assign $b_{1}=N$, and we have the models when $b_{2}=b_{3}=2 N-2$ shown in table 20. If $b_{2}=2 N-2$, $b_{3}=N$, then one needs to take

$$
\mathcal{W}=x^{N-1}+x t^{2}+z_{1} t+z_{2}^{p_{2}}+z_{3}^{p_{3}} t,
$$

add marginal terms to make it isolated, and then integrate out $z_{1}$ and $t$ (a similar procedure should be applied to the $b_{2}=b_{3}=N$ cases as well). We list the resulting singular equations and the data of the corresponding theories in table 20. Note that many of these models are equivalent to $E_{k}^{(n)}$ theories or to other models introduced above.

For $G=E_{6}$, we can choose either $b_{1}=9$ or 8 , and the models are shown in table 21 . Likewise, for $G=E_{7}$ we need to set $b_{1}=14$, and the corresponding models can be found in table 22. Finally, for $G=E_{8}$, we can choose either $b_{1}=24$ or 20 , as shown in table 23 .

\subsection{Matching the CB spectrum to the spectrum of the singularity}

We can provide detailed checks of the above results by comparing the $4 \mathrm{~d} \mathrm{CB}$ spectrum obtained from the threefold singularity in Type IIB $[8,36]$ to the CB spectrum of the individual $D_{p}^{b}(G)$ legs.

Let us illustrate this by presenting one example in more detail. Let us choose the first model in table 21. The trinion takes the form

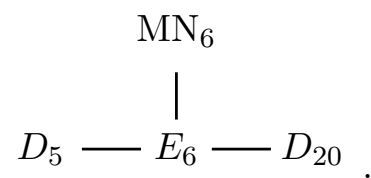

Here we used the fact that $D_{1}^{9}\left(E_{6}\right)$ is the $E_{6}$ MN theory. The three legs have the CB spectrum

$$
\begin{aligned}
D_{1}^{9}\left(E_{6}\right): \Delta= & \{3\}, \\
D_{5}^{12}\left(E_{6}\right): \Delta= & \left\{\frac{6}{5}, \frac{9}{5}, \frac{12}{5}, \frac{13}{5}, \frac{16}{5}, \frac{18}{5}, \frac{21}{5}, \frac{24}{5}, \frac{28}{5}, \frac{33}{5}, \frac{36}{5}, \frac{48}{5}\right\}, \\
D_{20}^{12}\left(E_{6}\right): \Delta= & \left\{\frac{6}{5}, \frac{6}{5}, \frac{6}{5}, \frac{7}{5}, \frac{7}{5}, \frac{7}{5}, \frac{9}{5}, \frac{9}{5}, \frac{9}{5}, 2,2, \frac{12}{5}, \frac{12}{5}, \frac{12}{5}, \frac{13}{5}, \frac{13}{5}, 3,3,3, \frac{16}{5}, \frac{16}{5},\right. \\
& \frac{18}{5}, \frac{18}{5}, \frac{18}{5}, \frac{19}{5}, \frac{19}{5}, \frac{21}{5}, \frac{21}{5}, \frac{21}{5}, \frac{22}{5}, \frac{22}{5}, \frac{24}{5}, \frac{24}{5}, \frac{24}{5}, 5, \frac{27}{5}, \frac{27}{5}, \frac{27}{5}, \frac{28}{5}, \\
& \left.6,6, \frac{31}{5}, \frac{33}{5}, \frac{33}{5}, \frac{34}{5}, \frac{36}{5}, \frac{36}{5}, \frac{37}{5}, \frac{39}{5}, \frac{39}{5}, \frac{42}{5}, \frac{42}{5}, 9, \frac{48}{5}, \frac{51}{5}, \frac{54}{5}, \frac{57}{5}\right\},
\end{aligned}
$$

the union of these spectra, plus the $E_{6}$ Casimirs, which give $\Delta=\{2,5,6,8,9,12\}$, result in the spectrum of the $D_{p}^{b}\left(E_{6}\right)$ trinion (7.11), which is a theory of rank $\widehat{r}=76$. On the other hand, the LG description of the trinion is

$$
\mathcal{W}=x^{3}+t^{4}+t z_{1}+z_{2}^{5}+z_{3}^{20} .
$$


Integrating out the massive fields $t$ and $z_{1}$, we obtain the threefold singularity

$$
y^{2}+x^{3}+z_{2}^{5}+z_{3}^{20}=0
$$

Its singularity spectrum precisely reproduce the CB spectrum spelled out above.

\section{S-dualities between $4 \mathrm{~d} D_{p}^{b}(G)$-trinions}

\subsection{S-dualities from geometric equivalence}

By comparing the results of sections 6 and 7 , we observe that, in a number of examples, the same singularity simultaneously describes more than one gauging of three $D_{p}^{b}(G)$ theories, sometimes involving different gauge groups $G$. The reason for this is mundane from the 2d LG perspective. By the $2 \mathrm{~d} / 4 \mathrm{~d}$ correspondence [67], the LG superpotential $\mathcal{W}(7.1)$ corresponds to a $G$-gauging of three $D_{p}^{b}(G)$ theories. It can then happen that the $\mathcal{W}$ for two distinct $D_{p}^{b}(G)$ trinions are equal, up to massive fields that can be decoupled. For simplicity, we have focussed on LG models with at most 4 non-trivial fields, that can be interpreted as canonical singularities $\mathbf{X}$ in Type IIB.

The consequence of this simple observation for the $4 \mathrm{~d} \mathcal{N}=2$ SCFTs $\mathscr{T}_{\mathbf{X}}^{4 \mathrm{~d}}$ is rather interesting. Since the $4 \mathrm{~d}$ theory is determined unambigiously by the singularity (or by $\mathcal{W}$ ), the distinct-looking $D_{p}^{b}(G)$ trinions associated to a single $\mathbf{X}$ are actually exactly equivalent. We have thus discovered a new type of S-duality among such $4 \mathrm{~d}$ SCFTs. The equivalent $D_{p}^{b}(G)$ trinions describe two S-duality frames of the same theory $\mathscr{T}_{\mathbf{X}}^{4 \mathrm{~d}}$, and the gauge groups manifest in each description become weakly coupled at different cusps of the conformal manifold. From the field-theoretic point of view, these dualities appear rather miraculous. By inspection of the list of theories in section 6 and section 7 , we then identify a large number of these S-dualities. We list them below and briefly comment on some of their properties. We hope to return to an in-depth analysis of these theories in the future.

The list of S-dualities can be simplified by observing that, for a fixed gauge group $G$, we have the following equivalence relation between $D_{p}^{b}(G)$ theories

$$
D_{p_{1}}^{b_{1}}(G) \equiv D_{p_{2}}^{b_{2}}(G) \quad \text { if } \quad \frac{b_{1}}{p_{1}}=\frac{b_{2}}{p_{2}}
$$

This follows from the geometric engineering of section 2.2, since the scaling dimensions of $D_{p}^{b}(G)$ only depend on $\frac{b}{p}$.

In order to describe the structure of the conformal manifold of $\mathscr{T}_{\mathbf{X}}^{4 \mathrm{~d}}$, we will need a class of isolated (i.e. with trivial conformal manifold) $4 \mathrm{~d}$ SCFTs dubbed $R_{2, N}$ (for $N$ odd) in [65]. This is a class $\mathcal{S}$ trinion with punctures $[k-1, k-1,1],[k-1, k-1,1]$ and $\left[1^{N}\right]$, with $N=2 k-1$. This theory has CB operators of dimension $3,5, \ldots, N-2, N$ and global symmetry $\mathrm{SO}(2 N+4)_{2 N} \times \mathrm{U}(1)$, where the subscript $2 N$ denotes the flavor central charge. We also notice that the $3 \mathrm{~d}$ mirrors (or magnetic quivers) of $R_{2, N}$ theories appear in (6.17), which tells us that $R_{2, N}$ is the dimensional reduction of the $5 \mathrm{~d}$ SCFT which UV completes $\mathrm{SU}(k)_{ \pm \frac{3}{2}}+(2 k+1) \boldsymbol{F}$. This family of theories is relevant because it arises in the 
description of the conformal manifold of $D_{2 n}(\mathrm{SO}(2 n+2))$ theories. More precisely, we find that $D_{2 n}(\mathrm{SO}(2 n+2))$ is equivalent to

$$
1-\mathrm{SU}(2)-\mathrm{SU}(3)-4 \leftarrow \mathrm{SU}(4) \rightarrow R_{2,5} \leftarrow \mathrm{SO}(8) \rightarrow \cdots \leftarrow \mathrm{SO}(2 n) \rightarrow R_{2,2 n-1},
$$

where the notation $A \leftarrow G \rightarrow B$ means that we glue together the SCFTs $A$ and $B$ by gauging a $G$ subgroup of their global symmetry.

\subsection{A list of new S-dualities}

We will now discuss in detail several examples of S-dualities we can obtain with this method. We find it convenient to organise the list according to $r$, the rank of the $5 \mathrm{~d} \operatorname{SCFT} \mathcal{T}_{\mathbf{X}}^{5 \mathrm{~d}}$ associated with the same singularity.

Rank-1 $\boldsymbol{E}_{\mathbf{8}}$ model. As our first example, the rank-1 $E_{8}$ theory in 5 d is described by the singularity

$$
x^{6}+z_{1}^{2}+z_{2}^{3}+z_{3}^{6}=0 .
$$

Modulo the trivial equivalences (8.1), there are actually two $D_{p}^{b}(G)$ trinions that describe the same theory $\mathscr{T}_{\mathbf{X}}^{4 \mathrm{~d}}$

$$
\mathscr{T}_{E_{8}}^{4 \mathrm{~d}}=\underbrace{2}_{6}
$$

In the first S-duality frame, which is fully Lagrangian, we clearly see that the conformal manifold has dimension 8. By using the equivalence (8.2) $D_{6}(\mathrm{SO}(8)) \equiv 1-\mathrm{SU}(2)-$ $\mathrm{SU}(3)-\mathrm{SU}(4) \rightarrow R_{2,5}$ and the fact that $D_{1}^{4}(\mathrm{SO}(8))$ is equivalent to $\mathrm{SU}(2) N_{f}=4 \mathrm{SQCD}$, we easily see that the $\mathrm{SO}(8)$ trinions as well have a eight-dimensional conformal manifold, in which the $\mathrm{SU}(5)$ and $\mathrm{SU}(6)$ groups of the unitary trinion are replaced by $\mathrm{SU}(2)$ and $\mathrm{SO}(8)$. In the $\mathrm{SO}(8)$ trinion description the theory is not fully Lagrangian, as it includes two copies of the strongly-coupled $R_{2,5}$ theory. Indeed the gauge couplings of the two trinions tend to zero at different cusps of the conformal manifold.

Rank-2 $\boldsymbol{E}_{\mathbf{6}}$ model. The singularity that realizes the rank- $2 E_{6} 5 \mathrm{~d}$ theory with $\left(r, f, d_{\mathrm{H}}\right)=$ $(2,6,23)$ leads in $4 \mathrm{~d}$ to the duality

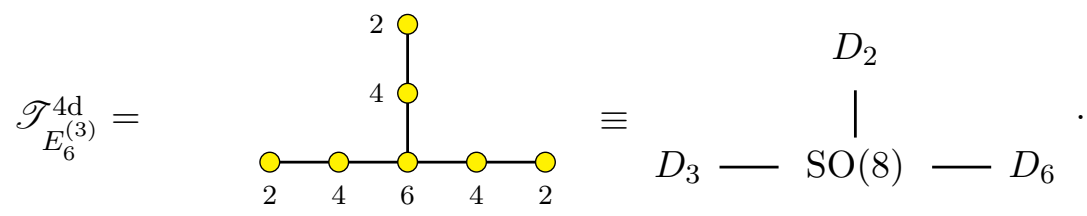

In both S-duality frames, the conformal manifold has dimension seven. In the special unitary trinion this is obvious, and in the $\mathrm{SO}(8)$ trinion this follows from the fact that $D_{2}(\mathrm{SO}(8))$ is isolated (it is the rank- $1 E_{6}$ theory), $D_{6}(\mathrm{SO}(8))$ has three marginal couplings, as we have already explained, and $D_{3}(\mathrm{SO}(8))$ is the Lagrangian theory $\mathrm{SO}(4)-\mathrm{Sp}(2)-4$. 
Rank-2 $\boldsymbol{E}_{\mathbf{7}}$ model. A similar result holds for the singularity that realizes the rank-2 $E_{7}$ theory with $\left(r, f, d_{\mathrm{H}}\right)=(2,7,35)$ :

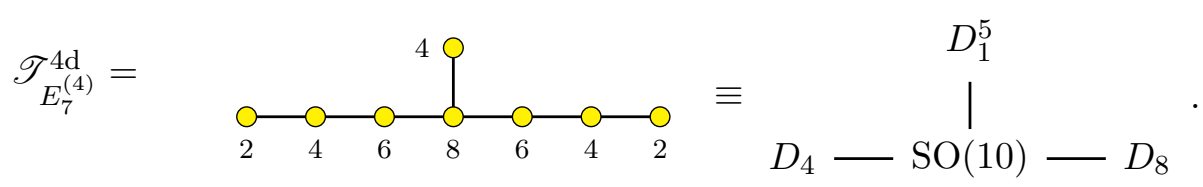

Once again we can match the dimension of the conformal manifold on the two sides. The special unitary side is easy to understand since the theory is Lagrangian, whereas the $\mathrm{SO}(10)$ trinion is more subtle: $D_{1}^{5}(\mathrm{SO}(10))$ is the rank- $1 E_{6}$ theory, $D_{8}(\mathrm{SO}(10)) \equiv R_{2,7} \leftarrow$ $\mathrm{SO}(8) \rightarrow D_{6}(\mathrm{SO}(8))$ from $(8.2)$ and $D_{4}(\mathrm{SO}(10))=1-\mathrm{SU}(2)-\mathrm{SO}(6)-\mathrm{Sp}(3)-5$.

Rank-2 $\boldsymbol{E}_{\mathbf{8}}$ model and triality. In the case of the $r=2 E_{8}$ theory, i.e. the hypersurface $x^{12}+z_{1}^{2}+z_{2}^{3}+z_{3}^{6}=0$, we interestingly find a triality

$$
\begin{aligned}
& \mathscr{T}_{E_{8}^{(6)}}^{4 \mathrm{~d}}={ }_{4}^{4} \\
& \equiv D_{3}-\operatorname{SO}(14)-D_{12} \equiv D_{6}-E_{6}-D_{12}{ }^{\mid}
\end{aligned}
$$

In the $\mathrm{SO}(14)$ trinion, the nine-dimensional conformal manifold arises as follows: $D_{1}^{7}(\mathrm{SO}(14))$ is isolated, being equivalent to $R_{2,5}$. $D_{3}(\mathrm{SO}(14))$ is equivalent to the Lagrangian theory $\mathrm{SO}(6)-\mathrm{Sp}(4)-7$ and finally we have $D_{12}(\mathrm{SO}(14))$, which fits in the family (8.2) and has a six-dimensional conformal manifold.

Sporadic model (2) with $\boldsymbol{r}=\mathbf{2}$. The singularity associated with the 5 d theory with $\left(r, f, d_{\mathrm{H}}\right)=(2,8,46)$ in table 11 leads to the following S-duality

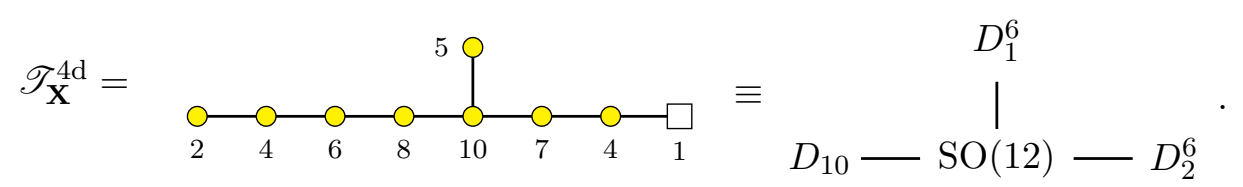

Rank-3 $\boldsymbol{E}_{\mathbf{7}}$ model. We also find an S-duality based on the $r=3 E_{7}$ theory



Rank-3 $\boldsymbol{E}_{\mathbf{8}}$ model. Likewise, the rank-3 $E_{8}$ theory leads to the S-duality

$$
\mathscr{T}_{E_{8}^{4(12)}}^{\mathrm{d}}=\underbrace{6}_{18}
$$


Rank-3 model. The singularity $x^{9}+y^{2}+z_{2}^{3}+z_{3}^{9}=0$ associated with the rank-3 theories appearing in table 20 and 22 , respectively, with $\left(r, f, d_{\mathrm{H}}\right)=(3,0,64)$, implies the duality

$$
\mathscr{T}_{\mathbf{X}}^{4 \mathrm{~d}}=D_{3}-\operatorname{SO}(20)-D_{9} \equiv D_{9}-E_{7}^{10}-D_{9}
$$

Notice that the $\mathrm{SO}(20)$ trinion is a fully Lagrangian SCFT, given by the orthosymplectic quiver

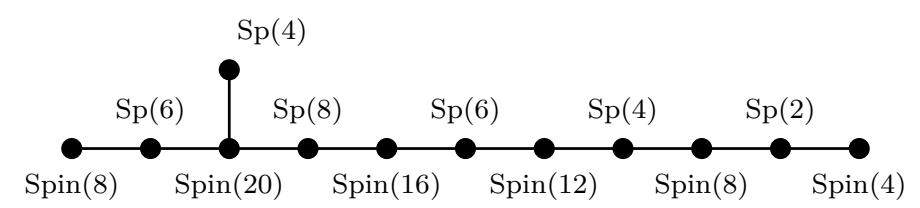

with a conformal manifold of dimension 13. This quiver has an electric one-form symmetry $\mathfrak{f}=\mathbb{Z}_{2}^{7}$, in agreement with the geometry. This is an interesting example of an S-duality that does not involve an $\mathrm{SU}(N)$ trinion.

We also find three curious examples associated with 5d SCFTs of rank larger than three:

Rank-4 model. The rank-4 theory with $\left(r, f, d_{\mathrm{H}}\right)=(4,5,40)$ appears in table 20 and in table 21 , corresponding to the singularity $t^{4}+y^{2}+z_{2}^{6}+z_{3}^{6}=0$ which results in the S-duality

$$
\mathscr{T}_{\mathbf{X}}^{4 \mathrm{~d}}=D_{D_{4}-\operatorname{SO}(14)-D_{6}}^{D_{1}^{7}} \equiv D_{6}-E_{6}-D_{6}
$$

The conformal manifold is of dimension 7 .

Rank-5 $\boldsymbol{E}_{\mathbf{8}}$ model. The rank-5 $E_{8}$ theory, associated to the singularity $x^{3}+y^{2}+z_{2}^{6}+z_{3}^{30}=$ 0 , leads to the duality

$$
\mathscr{T}_{E_{8}^{4(24)}}^{4 \mathrm{~d}}=\quad \sim_{30}^{10}
$$

Note that $D_{1}^{8}\left(E_{8}\right)$ is the rank-1 $E_{8} \mathrm{MN}$ theory. We thus find an S-dual Lagrangian theory of the $E_{8}$ gauging of the $E_{8} \mathrm{MN}$ theory (with the other two $D_{p}\left(E_{8}\right)$ tails appended, to total rank being $\widehat{r}=141$ ) as a $E_{8}$-shaped special unitary quiver.

Rank-6 model. Finally, the rank-6 theory with $\left(r, f, d_{\mathrm{H}}\right)=(6,0,77)$ appears in table 20 and table 23, corresponding to the singularity $x^{15}+y^{2}+z_{2}^{5}+z_{3}^{3} z_{2}=0$. This implies the duality

$$
\mathscr{T}_{\mathbf{X}}^{4 \mathrm{~d}}=D_{5}-\mathrm{SO}(32)-D_{2}^{16} \equiv D_{15}-E_{8}-D_{3}^{24} .
$$


We note that the $\mathrm{SO}(32)$ trinion is actually a Lagrangian theory

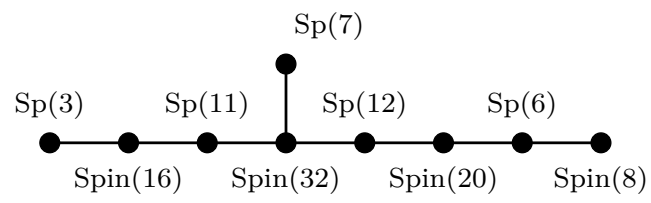

with a 9-dimensional conformal manifold and an electric one-form symmetry $\mathfrak{f}=\mathbb{Z}_{2}^{5}$, in perfect agreement with the geometry.

\section{Acknowledgments}

We thank Fabio Apruzzi, Lakshya Bhardwaj, Antoine Bourget, Stefano Cremonesi, Michele Del Zotto, Antonella Grassi, Amihay Hanany, Sheldon Katz, Horia Magureanu, Wolfger Peelaers and Timo Weigand for discussions. The work of SG, SSN, Y-NW, is supported by the ERC Consolidator Grant number 682608 "Higgs bundles: Supersymmetric Gauge Theories and Geometry (HIGGSBNDL)". SSN acknowledges support also from the Simons Foundation. CC is a Royal Society University Research Fellow and a Birmingham Fellow.

\section{A Resolutions of hypersurface canonical singularities}

In this section, we present the detailed resolution sequences and resolved geometry of the isolated hypersurface (IHS) equations. We use the notation and conventions of [106], see also [36]. In particular blowups are denoted by

$$
\left(x_{1}^{\left(i_{1}\right)}, x_{2}^{\left(i_{2}\right)}, x_{3}^{\left(i_{3}\right)}, x_{4}^{\left(i_{4}\right)} ; \delta\right),
$$

which blows up the locus $x_{1}=x_{2}=x_{3}=x_{4}=0$ with weights $i_{j}$ and the exceptional divisor is $\delta$. This blowup inserts a weighted projective space given by the weights. If there are not $\left(i_{j}\right)$ labels, the weights are 1 .

\section{A.1 $E_{n}^{(k)}$ theories}

For the $E_{n}^{(k)}$ theories with rank $r>0$, we review the resolution sequences in [36]. For $E_{6}^{(k)}$ theories with the singular equation

$$
x_{1}^{3}+x_{2}^{3}+x_{3}^{3}+x_{4}^{3+k}=0,
$$

the resolution sequence is

$$
\left(x_{1}, x_{2}, x_{3}, x_{4} ; \delta_{1}\right), \quad\left(x_{1}, x_{2}, x_{3}, \delta_{i} ; \delta_{i+1}\right)(i=1, \ldots, r-1) .
$$

We use $S_{i}$ to denote the exceptional divisor $\delta_{i}=0$, and the rank of this theory is $r=\left\lfloor\frac{k}{3}\right\rfloor+1$. The triple intersection numbers among the divisors $S_{1}, \ldots, S_{r}$ are

$$
\begin{aligned}
S_{1}^{3} & =S_{2}^{3}=\cdots=S_{r-1}^{3}=0, & S_{r}^{3} & =3 \\
S_{i}^{2} \cdot S_{i-1} & =-3, & S_{i-1}^{2} \cdot S_{i} & =3, \quad(i=2, \ldots, r) .
\end{aligned}
$$


For any $k \geq 3$, the divisors $S_{1}, \ldots, S_{r-1}$ are ruled surfaces over genus- 1 curves, and all the intersection curves $S_{i} \cdot S_{i-1}(i=2, \ldots, r)$ are smooth too. For the topology of the divisor $S_{r}$, we have the following cases:

1. $k=3 r-3$ : the divisor $S_{r}$ is a smooth $d P_{6}$.

2. $k=3 r-2$ : the resolved CY3 is smooth, but the divisor $S_{r}$ is a singular cubic surface with the following equation:

$$
x_{1}^{3}+x_{2}^{3}+x_{3}^{3}=0
$$

which has a point singularity at $x_{1}=x_{2}=x_{3}=0$.

3. $k=3 r-1$ : the resolved CY3 has a terminal singularity at the point $x_{1}=x_{2}=x_{3}=$ $\delta_{r}=0:$

$$
x_{1}^{3}+x_{2}^{3}+x_{3}^{3}+\delta_{r}^{2}=0 .
$$

The divisor $S_{r}$ also has the same singular equation in (A.5).

For $E_{7}^{(k)}$ theories (rank $r=\left\lfloor\frac{k}{4}\right\rfloor+1$ ) with the singular equation

$$
x_{1}^{2}+x_{2}^{4}+x_{3}^{4}+x_{4}^{4+k}=0
$$

the resolution sequence is

$$
\left(x_{1}^{(2)}, x_{2}^{(1)}, x_{3}^{(1)}, x_{4}^{(1)} ; \delta_{1}\right), \quad\left(x_{1}^{(2)}, x_{2}^{(1)}, x_{3}^{(1)}, \delta_{i}^{(1)} ; \delta_{i+1}\right), \quad i=1, \ldots, r-1 .
$$

The triple intersection numbers among the divisors $S_{1}, \ldots, S_{r}$ are

$$
\begin{aligned}
S_{1}^{3} & =S_{2}^{3}=\cdots=S_{r-1}^{3}=0, & S_{r}^{3} & =2 \\
S_{i}^{2} \cdot S_{i-1} & =-2, & S_{i-1}^{2} \cdot S_{i} & =2, \quad(i=2, \ldots, r) .
\end{aligned}
$$

For any $k \geq 4$, the divisors $S_{1}, \ldots, S_{r-1}$ are smooth genus-1 ruled surfaces. For the topology of the divisor $S_{r}$, we have the following cases:

1. $k=4 r-4:$ the divisor $S_{r}$ is a smooth $d P_{7}$.

2. $k=4 r-3:$ the resolved CY3 is smooth, but the divisor $S_{r}$ is a singular surface with the following equation:

$$
x_{1}^{2}+x_{2}^{4}+x_{3}^{4}=0,
$$

which has a point singularity at $x_{1}=x_{2}=x_{3}=0$.

3. $k=4 r-s(s=1,2)$ : the resolved CY3 has a terminal singularity at the point $x_{1}=x_{2}=x_{3}=\delta_{r}=0$ :

$$
x_{1}^{2}+x_{2}^{4}+x_{3}^{4}+\delta_{r}^{4-s}=0 .
$$

The divisor $S_{r}$ also has the same singular equation in (A.10). 
For $E_{8}^{(k)}$ theories (rank $r=\left\lfloor\frac{k}{6}\right\rfloor+1$ ) with the singular equation

$$
x_{1}^{2}+x_{2}^{3}+x_{3}^{6}+x_{4}^{6+k}=0,
$$

the resolution sequence is

$$
\left(x_{1}^{(3)}, x_{2}^{(2)}, x_{3}^{(1)}, x_{4}^{(1)} ; \delta_{1}\right), \quad\left(x_{1}^{(3)}, x_{2}^{(2)}, x_{3}^{(1)}, \delta_{i}^{(1)} ; \delta_{i+1}\right), \quad i=1, \ldots, r-1 .
$$

The triple intersection numbers among the divisors $S_{1}, \ldots, S_{r}$ are

$$
\begin{aligned}
S_{1}^{3} & =S_{2}^{3}=\cdots=S_{r-1}^{3}=0, \\
S_{i}^{2} \cdot S_{i-1} & =-1,
\end{aligned} \quad \begin{aligned}
S_{r}^{3} & =1 \\
S_{i-1}^{2} \cdot S_{i} & =1, \quad(i=2, \ldots, r) .
\end{aligned}
$$

For any $k \geq 6$, the divisors $S_{1}, \ldots, S_{r-1}$ are smooth genus- 1 ruled surfaces. For the topology of the divisor $S_{r}$, we have the following cases:

1. $k=6 r-6$ : the divisor $S_{r}$ is a smooth $d P_{8}$.

2. $k=6 r-5$ : the resolved CY3 is smooth, but the divisor $S_{r}$ is a singular surface with the following equation:

$$
x_{1}^{2}+x_{2}^{3}+x_{3}^{6}=0,
$$

which has a point singularity at $x_{1}=x_{2}=x_{3}=0$.

3. $k=6 r-s(s=1,2,3,4)$ : the resolved CY3 has a terminal singularity at the point $x_{1}=x_{2}=x_{3}=\delta_{r}=0$ :

$$
x_{1}^{2}+x_{2}^{3}+x_{3}^{6}+\delta_{r}^{6-s}=0 .
$$

The divisor $S_{r}$ also has the same singular equation in (A.15).

\section{A.2 13 sporadic trinion models}

We now determine the resolution for the 13 sporadic trinion theories from section 6 .

Sporadic theory \#(1). Starting from the singular equation

$$
x_{1}^{3}+x_{2}^{4}+x_{3}^{6}+x_{4}^{2} x_{3}=0,
$$

after the resolution

$$
\left(x_{1}, x_{2}, x_{3}, x_{4} ; \delta_{1}\right)
$$

there is still a residual terminal singularity at $x_{1}=x_{2}=\delta_{1}=x_{4}=0$ :

$$
x_{1}^{3}+x_{2}^{4} \delta_{1}+\delta_{1}^{3}+x_{4}^{2}=0,
$$

which is type II $(2,3,3,4)$, and equivalent to type I $(2,3,3,6)\left(E_{8}^{(-3)}\right)$. 
Sporadic theory \#(2). Starting from the singular equation

$$
x_{1}^{2}+x_{2}^{5}+x_{3}^{10}+x_{4}^{3} x_{3}=0
$$

the resolution sequence is

$$
\left(x_{1}^{(2)}, x_{2}^{(1)}, x_{3}^{(1)}, x_{4}^{(1)} ; \delta_{1}\right), \quad\left(x_{1}^{(3)}, x_{2}^{(1)}, x_{4}^{(2)}, \delta_{1}^{(1)} ; \delta_{2}\right) .
$$

After the resolution, the equation becomes

$$
x_{1}^{2}+x_{2}^{5} \delta_{1}+x_{3}^{10} \delta_{1}^{6}+x_{4}^{3} x_{3}=0,
$$

the divisor $\delta_{1}=0$ is singular at $x_{1}=x_{4}=0$ :

$$
\delta_{1}=0: x_{1}^{2}+x_{4}^{3} x_{3}=0 .
$$

The detailed geometry was already discussed in [36], which eventually gives rise to $5 \mathrm{~d}$ $\mathrm{SU}(2)-\mathrm{SU}(2)-5 \boldsymbol{F}$ gauge theory after a flop.

Sporadic theory \#(3). Starting from the singular equation

$$
x_{1}^{3}+x_{2}^{5}+x_{3}^{15}+x_{4}^{2} x_{3}=0,
$$

the resolution sequence is

$$
\left(x_{1}, x_{2}, x_{3}, x_{4} ; \delta_{1}\right), \quad\left(x_{1}^{(2)}, x_{2}^{(1)}, x_{4}^{(3)}, \delta_{1}^{(1)} ; \delta_{3}\right), \quad\left(x_{1}^{(2)}, x_{2}^{(1)}, x_{4}^{(3)}, \delta_{3}^{(1)} ; \delta_{4}\right), \quad\left(x_{1}, x_{4}, \delta_{1} ; \delta_{2}\right) .
$$

After the resolution, the equation becomes

$$
\delta_{2} x_{1}^{3}+\delta_{1}^{2} \delta_{3} x_{2}^{5}+\delta_{1}^{12} \delta_{2}^{10} \delta_{3}^{6} x_{3}^{15}+x_{3} x_{4}^{2}=0 .
$$

The divisor $\delta_{3}=0$ is singular $x_{1}=x_{4}=0$

$$
\delta_{3}=0: \delta_{2} x_{1}^{3}+x_{3} x_{4}^{2}=0 .
$$

Sporadic theory \#(4). Starting from the singular equation

$$
x_{1}^{3}+x_{2}^{5}+x_{3}^{2} x_{2}+x_{4}^{3} x_{2}=0,
$$

after the resolution

$$
\left(x_{1}, x_{2}, x_{3}, x_{4} ; \delta_{1}\right)
$$

there is a residual terminal singularity at $x_{1}=x_{3}=x_{4}=\delta_{1}=0$ :

$$
x_{1}^{3}+\delta_{1}^{2}+x_{3}^{2}+x_{4}^{3} \delta_{1}+x_{1} x_{3} x_{4}=0,
$$

which is Type I $(2,2,3,6)$ (corresponding to $\left.E_{8}^{(-4)}\right)$, and another terminal singularity at $x_{1}=x_{2}=x_{3}=\delta_{1}=0$ :

$$
x_{1}^{3}+x_{2}^{5} \delta_{1}^{2}+x_{3}^{2} x_{2}+x_{2} \delta_{1}+x_{1} x_{3}=0,
$$

which is the conifold singularity. 
Sporadic theory \#(5). Starting from the singular equation

$$
x_{1}^{2}+x_{2}^{7}+x_{3}^{3} x_{2}+x_{4}^{4} x_{2}+x_{3}^{2} x_{4}^{2}=0,
$$

after the resolution

$$
\left(x_{1}^{(2)}, x_{2}^{(1)}, x_{3}^{(1)}, x_{4}^{(1)} ; \delta_{1}\right),
$$

there is a residual terminal singularity at $x_{1}=x_{2}=x_{3}=\delta_{1}=0$ :

$$
x_{1}^{2}+x_{2}^{7} \delta_{1}^{3}+x_{3}^{3} x_{2}+x_{2} \delta_{1}+x_{3}^{2}=0,
$$

which is a conifold singularity. There is also a residual terminal singularity at $x_{1}=x_{3}=$ $x_{4}=\delta_{1}=0$ :

$$
x_{1}^{2}+\delta_{1}^{3}+x_{3}^{3}+x_{4}^{4} \delta_{1}+x_{3}^{2} x_{4}^{2}=0,
$$

which is type $\mathrm{I}(2,3,3,6)$, the same as $E_{8}^{(-3)}$.

Sporadic theory \#(6). From the singular equation

$$
x_{1}^{3}+x_{2}^{9}+x_{3}^{2} x_{2}+x_{4}^{4} x_{2}=0,
$$

the resolution sequence is

$$
\left(x_{1}, x_{2}, x_{3}, x_{4} ; \delta_{1}\right), \quad\left(x_{1}^{(2)}, x_{3}^{(3)}, x_{4}^{(1)}, \delta_{1}^{(1)} ; \delta_{3}\right), \quad\left(x_{1}, x_{3}, \delta_{1} ; \delta_{2}\right) .
$$

The resolved equation and exceptional divisors are smooth.

Sporadic theory \#(7). From the singular equation

$$
x_{1}^{4}+x_{2}^{10}+x_{3}^{2} x_{2}+x_{4}^{3} x_{2}+x 1 x 3 x 4=0,
$$

after the resolution

$$
\left(x_{1}, x_{2}, x_{3}, x_{4} ; \delta_{1}\right), \quad\left(x_{1}^{(1)}, x_{3}^{(2)}, x_{4}^{(1)}, \delta_{1}^{(1)} ; \delta_{3}\right), \quad\left(x_{3}, \delta_{1} ; \delta_{2}\right),
$$

the equation has a terminal singularity at $x_{1}=x_{3}=x_{4}=\delta_{3}=0$ :

$$
x_{1}^{4} \delta_{3}+\delta_{3}^{3}+x_{3}^{2}+x_{4}^{3}+x_{1} x_{3} x_{4}=0 .
$$

It is type $\mathrm{I}(2,3,3,6)$, which is the same as $E_{8}^{(-3)}$.

Sporadic theory \#(8). From the singular equation

$$
x_{1}^{2}+x_{2}^{16}+x_{3}^{3} x_{2}+x_{4}^{5} x_{2}+x_{3}^{2} x_{4}^{2}=0,
$$

the resolution sequence is

$$
\left(x_{1}^{(2)}, x_{2}^{(1)}, x_{3}^{(1)}, x_{4}^{(1)} ; \delta_{1}\right), \quad\left(x_{1}^{(3)}, x_{3}^{(2)}, x_{4}^{(1)}, \delta_{1}^{(1)} ; \delta_{3}\right), \quad\left(x_{1}^{(3)}, x_{3}^{(2)}, x_{4}^{(1)}, \delta_{3}^{(1)} ; \delta_{4}\right), \quad\left(x_{3}, \delta_{1} ; \delta_{2}\right) .
$$

The resolved equation is

$$
x_{1}^{2}+\delta_{1}^{12} \delta_{2}^{10} \delta_{3}^{6} x_{2}^{16}+\delta_{2} x_{2} x_{3}^{3}+\delta_{1}^{2} \delta_{3} x_{2} x_{4}^{5}+x_{3}^{2} x_{4}^{2}=0,
$$

and the divisor $\delta_{3}=0$ is singular at $x_{1}=x_{3}=0$ :

$$
x_{1}^{2}+\delta_{2} x_{2} x_{3}^{3}+x_{3}^{2} x_{4}^{2}=0 .
$$


Sporadic theory \#(9). From the singular equation

$$
x_{1}^{3}+x_{2}^{21}+x_{3}^{2} x_{2}+x_{4}^{5} x_{2}+x_{1} x_{3} x_{4}=0,
$$

the resolution sequence is

$$
\begin{aligned}
& \left(x_{1}, x_{2}, x_{3}, x_{4} ; \delta_{1}\right) \\
& \left(x_{1}^{(2)}, x_{3}^{(3)}, x_{4}^{(1)}, \delta_{1}^{(1)} ; \delta_{4}\right) \\
& \left(x_{1}^{(2)}, x_{3}^{(3)}, x_{4}^{(1)}, \delta_{4}^{(1)} ; \delta_{6}\right) \\
& \left(x_{1}^{(2)}, x_{3}^{(3)}, x_{4}^{(1)}, \delta_{6}^{(1)} ; \delta_{7}\right) \\
& \left(x_{1}, x_{3}, \delta_{1} ; \delta_{2}\right), \quad\left(x_{3}, \delta_{2} ; \delta_{3}\right), \quad\left(x_{1}, x_{3}, \delta_{4} ; \delta_{5}\right) .
\end{aligned}
$$

The resolved equation is

$$
\delta_{2} \delta_{5} x_{1}^{3}+x_{1} x_{3} x_{4}+\delta_{1}^{18} \delta_{2}^{16} \delta_{3}^{15} \delta_{4}^{12} \delta_{5}^{10} \delta_{6}^{6} x_{2}^{21}+\delta_{3} x_{2} x_{3}^{2}+\delta_{1}^{3} \delta_{2} \delta_{4}^{2} \delta_{6} x_{2} x_{4}^{5}=0 .
$$

The divisor $\delta_{6}=0$ is singular at $x_{1}=x_{3}=0$ :

$$
\delta_{6}=0: \quad \delta_{2} \delta_{5} x_{1}^{3}+x_{1} x_{3} x_{4}+\delta_{3} x_{2} x_{3}^{2}=0 .
$$

Sporadic theory \#(10). From the singular equation

$$
x_{1}^{5}+x_{2}^{25}+x_{3}^{2} x_{2}+x_{4}^{3} x_{2}=0
$$

the resolution sequence is

$$
\begin{aligned}
& \left(x_{1}, x_{2}, x_{3}, x_{4} ; \delta_{1}\right), \quad\left(x_{1}^{(1)}, x_{3}^{(2)}, x_{4}^{(1)}, \delta_{1}^{(1)} ; \delta_{4}\right), \quad\left(x_{1}^{(1)}, x_{3}^{(3)}, x_{4}^{(2)}, \delta_{4}^{(1)} ; \delta_{7}\right), \\
& \left(x_{1}^{(1)}, x_{3}^{(3)}, x_{4}^{(2)}, \delta_{7}^{(1)} ; \delta_{9}\right), \quad\left(x_{1}^{(1)}, x_{3}^{(3)}, x_{4}^{(2)}, \delta_{9}^{(1)} ; \delta_{10}\right), \\
& \left(x_{3}, \delta_{1} ; \delta_{2}\right), \quad\left(x_{4}, \delta_{2} ; \delta_{3}\right), \quad\left(x_{3}, x_{4}, \delta_{4} ; \delta_{5}\right), \quad\left(x_{3}, \delta_{5} ; \delta_{6}\right), \quad\left(x_{3}, x_{4}, \delta_{7} ; \delta_{8}\right) .
\end{aligned}
$$

The resolved equation is

$$
\delta_{1}^{2} \delta_{2} \delta_{4}^{3} \delta_{5} \delta_{7}^{2} \delta_{9} x_{1}^{5}+x_{1} x_{3} x_{4}+\delta_{1}^{22} \delta_{2}^{21} \delta_{3}^{20} \delta_{4}^{18} \delta_{5}^{16} \delta_{6}^{15} \delta_{7}^{12} \delta_{8}^{10} \delta_{9}^{6} x_{2}^{2} 5+\delta_{2} \delta_{6} x_{2} x_{3}^{2}+\delta_{1} \delta_{3}^{2} \delta_{5} \delta_{8} x_{2} x_{4}^{3}=0
$$

The divisor $\delta_{9}=0$ is singular at $x_{3}=x_{4}=0$ :

$$
\delta_{6}=0: x_{1} x_{3} x_{4}+\delta_{2} \delta_{6} x_{2} x_{3}^{2}+\delta_{1} \delta_{3}^{2} \delta_{5} \delta_{8} x_{2} x_{4}^{3}=0 .
$$

Sporadic theory \#(11). From the singular equation

$$
x_{1}^{7}+x_{2}^{2} x_{1}+x_{3}^{3} x_{1}+x_{4}^{3} x_{1}+x_{2} x_{3}^{2}+x_{2} x_{4}^{2}+x_{2} x_{3} x_{4}=0,
$$

the resolution sequence is

$$
\left(x_{1}, x_{2}, x_{3}, x_{4} ; \delta_{1}\right), \quad\left(x_{2}^{(2)}, x_{3}^{(1)}, x_{4}^{(1)}, \delta_{1}^{(1)} ; \delta_{3}\right), \quad\left(x_{2}, \delta_{1} ; \delta_{2}\right) .
$$

The resolved equation and exceptional divisors are smooth. 
Sporadic theory \#(12). From the singular equation

$$
x_{1}^{13}+x_{2}^{2} x_{1}+x_{3}^{3} x_{1}+x_{4}^{4} x_{1}+x_{3} x_{4}^{3}+2 x_{2} x_{3} x_{4}=0,
$$

the resolution sequence is

$$
\begin{aligned}
& \left(x_{1}, x_{2}, x_{3}, x_{4} ; \delta_{1}\right) \\
& \left(x_{2}^{(2)}, x_{3}^{(1)}, x_{4}^{(1)}, \delta_{1}^{(1)} ; \delta_{4}\right) \\
& \left(x_{2}^{(3)}, x_{2}^{(2)}, x_{4}^{(1)}, \delta_{4}^{(1)} ; \delta_{6}\right) \\
& \left(x_{2}, \delta_{1} ; \delta_{2}\right), \quad\left(x_{3}, \delta_{2} ; \delta_{3}\right), \quad\left(x_{2}, x_{3}, \delta_{4} ; \delta_{5}\right) .
\end{aligned}
$$

The resolved equation and exceptional divisors are smooth.

Sporadic theory \#(13). From the singular equation

$$
x_{1}^{31}+x_{2}^{2} x_{1}+x_{3}^{3} x_{1}+x_{4}^{5} x_{1}+3 x_{2} x_{3} x_{4}=0,
$$

the resolution sequence is

$$
\begin{aligned}
& \left(x_{1}, x_{2}, x_{3}, x_{4} ; \delta_{1}\right), \quad\left(x_{2}^{(2)}, x_{3}^{(1)}, x_{4}^{(1)}, \delta_{1}^{(1)} ; \delta_{5}\right), \quad\left(x_{2}^{(3)}, x_{2}^{(2)}, x_{4}^{(1)}, \delta_{5}^{(1)} ; \delta_{9}\right) \\
& \left(x_{2}^{(3)}, x_{2}^{(2)}, x_{4}^{(1)}, \delta_{9}^{(1)} ; \delta_{12}\right), \quad\left(x_{2}^{(3)}, x_{2}^{(2)}, x_{4}^{(1)}, \delta_{12}^{(1)} ; \delta_{14}\right), \quad\left(x_{2}^{(3)}, x_{2}^{(2)}, x_{4}^{(1)}, \delta_{14}^{(1)} ; \delta_{15}\right) \\
& \left(x_{2}, \delta_{1} ; \delta_{2}\right), \quad\left(x_{3}, \delta_{2} ; \delta_{3}\right), \quad\left(x_{2}, \delta_{3} ; \delta_{4}\right), \\
& \left(x_{2}, x_{3}, \delta_{5} ; \delta_{6}\right), \quad\left(x_{2}, \delta_{6} ; \delta_{7}\right), \quad\left(x_{3}, \delta_{7} ; \delta_{8}\right), \\
& \left(x_{2}, x_{3}, \delta_{9} ; \delta_{10}\right), \quad\left(x_{2}, \delta_{10} ; \delta_{11}\right), \quad\left(x_{2}, x_{3}, \delta_{12} ; \delta_{13}\right) .
\end{aligned}
$$

The resolved equation is

$$
\begin{aligned}
& \delta_{1}^{28} \delta_{2}^{27} \delta_{3}^{26} \delta_{4}^{25} \delta_{5}^{24} \delta_{6}^{22} \delta_{7}^{21} \delta_{8}^{20} \delta_{9}^{18} \delta_{10}^{16} \delta_{11}^{15} \delta_{12}^{12} \delta_{13}^{10} \delta_{14}^{6} x_{1}^{31}+\delta_{2} \delta_{4} \delta_{7} \delta_{11} x_{1} x_{2}^{2}+ \\
& \delta_{1} \delta_{3}^{2} \delta_{4} \delta_{6} \delta_{8}^{2} \delta_{10} \delta_{13} x_{1} x_{3}^{3}+\delta_{1}^{3} \delta_{2}^{2} \delta_{3} \delta_{5}^{4} \delta_{6}^{2} \delta_{7} \delta_{9}^{3} \delta_{10} \delta_{12}^{2} \delta_{14} x_{1} x_{4}^{5}+3 x_{2} x_{3} x_{4}=0 .
\end{aligned}
$$

The divisor $\delta_{14}=0$ is singular at $x_{2}=x_{3}=0$ :

$$
\delta_{14}=0: \delta_{2} \delta_{4} \delta_{7} \delta_{11} x_{1} x_{2}^{2}+\delta_{1} \delta_{3}^{2} \delta_{4} \delta_{6} \delta_{8}^{2} \delta_{10} \delta_{13} x_{1} x_{3}^{3}+3 x_{2} x_{3} x_{4}=0 .
$$

\section{A.3 Type $\operatorname{VIII}_{2}(N, N, 2,2)$ series}

Consider the type $\mathrm{VIII}_{2}(N, N, 2,2)$ series in table 10 , which are equivalent to type $\operatorname{IV}(N, 2, N, 2)$. We now resolve these models.

$\boldsymbol{N}=\mathbf{2 k}-\mathbf{1}$. Start with the singular equation

$$
x_{1}^{2 k-1}+x_{2}^{2 k-1}+x_{1} x_{3}^{2}+x_{1} x_{4}^{2}+x_{2} x_{3} x_{4}=0,
$$

the resolution sequence is

$$
\left(x_{1}, x_{2}, x_{3}, x_{4} ; \delta_{1}\right), \quad\left(x_{3}, x_{4}, \delta_{j} ; \delta_{j+1}\right), \quad j=1, \ldots, k-2 .
$$

The resolved CY3 and divisors are all smooth. 
$\boldsymbol{N}=\mathbf{2} \boldsymbol{k} . \quad$ Start with the singular equation

$$
x_{1}^{2 k}+x_{2}^{2 k}+x_{1} x_{3}^{2}+x_{1} x_{4}^{2}+x_{2} x_{3} x_{4}=0
$$

the resolution sequence is

$$
\left(x_{1}, x_{2}, x_{3}, x_{4} ; \delta_{1}\right), \quad\left(x_{3}, x_{4}, \delta_{j} ; \delta_{j+1}\right), \quad j=1, \ldots, k-2 .
$$

The resolved CY3 is

$$
\left(x_{1}^{2 k}+x_{2}^{2 k}\right) \prod_{i=1}^{k-1} \delta_{i}^{2 k-2 i-1}+x_{1} x_{3}^{2}+x_{1} x_{4}^{2}+x_{2} x_{3} x_{4}=0 .
$$

There are in total $2 k$ conifold singularities at the loci

$$
\delta_{k-1}=x_{3}=x_{4}=x_{1}+x_{2} e^{\frac{\pi(2 j+1)}{2 k}}=0, \quad j=1, \ldots, 2 k .
$$

\section{B Proof of the completeness of the $\mathrm{SU}(N)$ trinions}

In this section, we prove the completeness of our list of solutions to the conditions (6.6), (6.11) and (6.24) in section 6.

First of all, it is trivial to see that when $p_{1}, p_{2}, p_{3} \geq 3$, the l.h.s. of (6.6), (6.11), (6.24) is always strictly smaller than the right hand side. Hence one of $p_{\alpha}$ 's has to be smaller than 3 (equal to 2 ). We start with the equation (6.6)

$$
\frac{N}{p_{1}}+\frac{N}{p_{2}}+\frac{N-1}{p_{3}}=N
$$

we assume that $p_{1} \leq p_{2}$.

If $p_{1}=2$, we have

$$
\frac{N}{p_{2}}+\frac{N-1}{p_{3}}=\frac{N}{2} .
$$

If $p_{2}, p_{3}>3$, then $\frac{N}{p_{2}}+\frac{N-1}{p_{3}}<N / 2$, and the above equation cannot hold. If $p_{2}=3$, we have

$$
p_{3}=\frac{6 N-6}{N}<6
$$

which leads to sporadic solutions $\left(p_{1}, p_{2}, p_{3}\right)=(2,3,3),(2,3,4),(2,3,5)$.

On the other hand, if $p_{3}=2$, we have the infinite sequence $\left(p_{1}, p_{2}, p_{3}\right)=(2,2 N, 2)$. If $p_{3}=3$, we have

$$
p_{2}=\frac{6 N}{N+2}<6 .
$$

This leads to sporadic solutions $\left(p_{1}, p_{2}, p_{3}\right)=(2,3,3),(2,4,3),(2,5,3)$.

Finally, if $p_{3}=2$ and $p_{1}>2$, we have

$$
\frac{1}{p_{1}}+\frac{1}{p_{2}}=\frac{N+1}{2 N} .
$$


When $p_{1}, p_{2}>3$, this equation cannot hold. Hence one has to set $p_{1}=3$ and

$$
p_{2}=\frac{6 N}{N+3}<6 .
$$

It leads to sporadic solutions $\left(p_{1}, p_{2}, p_{3}\right)=(3,3,2),(3,4,2),(3,5,2)$. This concludes the enumeration of solutions to (6.6).

Next we consider (6.11)

$$
\frac{N}{p_{1}}+\frac{N-1}{p_{2}}+\frac{N-1}{p_{3}}=N,
$$

we assume that $p_{2} \leq p_{3}$. Then if $p_{1}=2$, we have

$$
\frac{1}{p_{2}}+\frac{1}{p_{3}}=\frac{N}{2(N-1)}>\frac{1}{2} .
$$

If $p_{2}, p_{3}>3$, then $\frac{1}{p_{2}}+\frac{1}{p_{3}} \leq 1 / 2$ and the above inequality cannot be satisfied. So we have to set $p_{2}=2$ or $p_{2}=3$. For the first case, we get the infinite sequence $\left(p_{1}, p_{2}, p_{3}\right)=$ $(2,2,2 N-2)$. For the second case, we have

$$
p_{3}=\frac{6 N-6}{N+2}<6 .
$$

Thus we have the sporadic solutions $\left(p_{1}, p_{2}, p_{3}\right)=(2,3,3),(2,3,4),(2,3,5)$.

If $p_{1}>2$ and $p_{2}=2$, then we have

$$
\frac{N}{p_{1}}+\frac{N-1}{p_{3}}=\frac{N+1}{2} .
$$

It is easy to see that if $p_{1}, p_{3}>3$, the l.h.s. above is strictly smaller than the r.h.s. If $p_{1}=3$, then we have

$$
p_{3}=\frac{6 N-6}{N+3}<6 .
$$

There are the sporadic solutions $\left(p_{1}, p_{2}, p_{3}\right)=(3,2,3),(3,2,4),(3,2,5)$. If $p_{3}=2$, we have the infinite sequence $\left(p_{1}, p_{2}, p_{3}\right)=(N, 2,2)$. If $p_{3}=3$, we have

$$
p_{1}=\frac{6 N}{N+5}<6 .
$$

This leads to the sporadic solutions $\left(p_{1}, p_{2}, p_{3}\right)=(3,2,3),(4,2,3),(5,2,3)$.

For (6.24)

$$
\frac{N-1}{p_{1}}+\frac{N-1}{p_{2}}+\frac{N-1}{p_{3}}=N
$$

we assume that $p_{1} \leq p_{2} \leq p_{3}$, and hence we have to set $p_{1}=2$. Now the equation is simplified to

$$
\frac{1}{p_{2}}+\frac{1}{p_{3}}=\frac{N+1}{2 N-2}>\frac{1}{2} .
$$

If $p_{2}, p_{3}>3$, the above inequality cannot be satisfied. So if $p_{2}=2$, we have the infinite sequence $\left(p_{1}, p_{2}, p_{3}\right)=(2,2, N-1)$. If $p_{2}=3$, we have the equation

$$
p_{3}=\frac{6 N-6}{N+5}<6
$$

which leads to the sporadic solutions $\left(p_{1}, p_{2}, p_{3}\right)=(2,3,3),(2,3,4),(2,3,5)$.

This concludes the enumeration of solutions. 


\section{Tables of trinions with $G=D, E$}

In this appendix we summarize the trinion theories for general $G=D, E$ found in section 7 . These are given in the following tables 15, 16, 17, 18, 19, 20, 21, 22, 23.

\begin{tabular}{|c|c|c|c|c|c|c|c|c|}
\hline $\mathrm{SO}(2 N): N$ & $\left(p_{2}, p_{3}\right)$ & $r$ & $f$ & $d_{\mathrm{H}}$ & $\widehat{r}$ & $\widehat{d}_{\mathrm{H}}$ & $b_{3}$ & $\mathfrak{f}$ \\
\hline $2 k$ & $(4,4)$ & $3 k-3$ & 6 & $9 k+3$ & $9 k-3$ & $3 k+3$ & 0 & 0 \\
$4 k+1$ & $(4,4)$ & $6 k-3$ & 3 & $18 k+6$ & $18 k+3$ & $6 k$ & 6 & $\mathbb{Z}_{2}^{3}$ \\
$4 k+3$ & $(4,4)$ & $6 k+1$ & 7 & $18 k+17$ & $18 k+10$ & $6 k+8$ & 0 & 0 \\
$6 k$ & $(3,6)$ & $6 k-2$ & 4 & $30 k+2$ & $30 k-2$ & $6 k+2$ & 0 & 0 \\
$6 k+1$ & $(3,6)$ & $6 k-2$ & 2 & $30 k+6$ & $30 k+4$ & $6 k$ & 8 & $\mathbb{Z}_{2}^{4}$ \\
$6 k+2$ & $(3,6)$ & $6 k$ & 4 & $30 k+12$ & $30 k+8$ & $6 k+4$ & 0 & 0 \\
$6 k+3$ & $(3,6)$ & $6 k$ & 2 & $30 k+16$ & $30 k+14$ & $6 k+2$ & 0 & $\mathbb{Z}_{2}$ \\
$6 k+4$ & $(3,6)$ & $6 k+2$ & 6 & $30 k+23$ & $30 k+17$ & $6 k+8$ & 2 & $\mathbb{Z}_{2}$ \\
$6 k+5$ & $(3,6)$ & $6 k+2$ & 2 & $30 k+26$ & $30 k+24$ & $6 k+4$ & 0 & $\mathbb{Z}_{2}$ \\
\hline
\end{tabular}

\begin{tabular}{|c|c|c|c|c|c|c|c|c|c|}
\hline$G$ & $\left(p_{2}, p_{3}\right)$ & $F$ & $r$ & $f$ & $d_{\mathrm{H}}$ & $\widehat{r}$ & $\widehat{d}_{\mathrm{H}}$ & $b_{3}$ & $\mathfrak{f}$ \\
\hline$E_{6}$ & $(4,4)$ & $x^{3}+t^{4}+z_{2}^{4}+z_{3}^{4}$ & 3 & 0 & 27 & 27 & 3 & 12 & $\mathbb{Z}_{3}^{3}$ \\
$E_{6}$ & $(3,6)$ & $x^{3}+t^{4}+z_{2}^{3}+z_{3}^{6}$ & 5 & 4 & 32 & 28 & 9 & 2 & $\mathbb{Z}_{2}$ \\
$E_{7}$ & $(4,4)$ & $x^{3}+x t^{3}+z_{2}^{4}+z_{3}^{4}$ & 9 & 3 & 33 & 30 & 12 & 0 & 0 \\
$E_{7}$ & $(3,6)$ & $x^{3}+x t^{3}+z_{2}^{3}+z_{3}^{6}$ & 8 & 2 & 36 & 34 & 10 & 4 & $\mathbb{Z}_{3}$ \\
$E_{8}$ & $(4,4)$ & $x^{3}+t^{5}+z_{2}^{4}+z_{3}^{4}$ & 12 & 0 & 36 & 36 & 12 & 0 & 0 \\
$E_{8}$ & $(3,6)$ & $x^{3}+t^{5}+z_{2}^{3}+z_{3}^{6}$ & 12 & 0 & 40 & 40 & 12 & 8 & $\mathbb{Z}_{5}$ \\
\hline
\end{tabular}

Table 15. Trinion models of the type described in section 7.2.1. with $b_{2}=b_{3}=h^{\vee}$.

\begin{tabular}{|c|c|cc|c|ccc|cc|cc|}
\hline$\left(b_{2}, b_{3}\right)$ & $G$ & $p_{2}$ & $p_{3}$ & $F$ & $r$ & $f$ & $d_{\mathrm{H}}$ & $\widehat{r}$ & $\widehat{d}_{\mathrm{H}}$ & $b_{3}$ & $\mathfrak{f}$ \\
\hline$(2 N-2, N)$ & $\mathrm{SO}(8)$ & 3 & 4 & $x^{3}+x t^{2}+z_{2}^{3}+z_{3}^{4} t$ & 2 & 6 & 23 & 17 & 8 & 2 & $\mathbb{Z}_{2}$ \\
& $\mathrm{SO}(8)$ & 6 & 2 & $x^{3}+x t^{2}+z_{2}^{6}+z_{3}^{2} t$ & 2 & 6 & 23 & 17 & 8 & 2 & $\mathbb{Z}_{2}$ \\
& $\mathrm{SO}(12)$ & 5 & 2 & $x^{5}+x t^{2}+z_{2}^{5}+z_{3}^{2} t$ & 4 & 4 & 30 & 26 & 8 & 4 & $\mathbb{Z}_{2}^{2}$ \\
\hline$(N, N)$ & $\mathrm{SO}(8)$ & 2 & 4 & $x^{3}+x t^{2}+z_{2}^{2} t+z_{3}^{4} t+z_{3}^{6}$ & 2 & 6 & 23 & 17 & 8 & 2 & $\mathbb{Z}_{2}$ \\
& $\mathrm{SO}(12)$ & 2 & 3 & $x^{5}+x t^{2}+z_{2}^{2} t+z_{3}^{3} t+z_{3}^{5}$ & 4 & 4 & 30 & 26 & 8 & 4 & $\mathbb{Z}_{2}^{2}$ \\
\hline
\end{tabular}

Table 16. Sporadic models for $G=\mathrm{SO}(N)$. Note that there are only two inequivalent theories in the table above, and the one with $r=2$ is the same as the rank- $2 E_{6}$ theory. 


\begin{tabular}{|c|c|cc|c|ccc|cc|cc|}
\hline$\left(b_{2}, b_{3}\right)$ & $G$ & $p_{2}$ & $p_{3}$ & $F$ & $r$ & $f$ & $d_{\mathrm{H}}$ & $\widehat{r}$ & $\widehat{d}_{\mathrm{H}}$ & $b_{3}$ & $\mathfrak{f}$ \\
\hline$(12,9)$ & $E_{6}$ & 4 & 3 & $x^{3}+t^{4}+z_{2}^{4}+z_{3}^{3} t$ & 3 & 0 & 27 & 27 & 3 & 12 & $\mathbb{Z}_{3}^{3}$ \\
& $E_{6}$ & 8 & 2 & $x^{3}+t^{4}+z_{2}^{8}+z_{3}^{2} t$ & 3 & 0 & 35 & 35 & 3 & 4 & $\mathbb{Z}_{3}^{2}$ \\
\hline$(12,8)$ & $E_{6}$ & 3 & 4 & $x^{3}+t^{4}+z_{2}^{3}+z_{3}^{4} x$ & 5 & 4 & 32 & 28 & 9 & 2 & $\mathbb{Z}_{2}$ \\
& $E_{6}$ & 6 & 2 & $x^{3}+t^{4}+z_{2}^{6}+z_{3}^{2} x$ & 5 & 4 & 32 & 28 & 9 & 2 & $\mathbb{Z}_{2}$ \\
\hline$(8,8)$ & $E_{6}$ & 2 & 4 & $x^{3}+t^{4}+z_{2}^{2} x+z_{3}^{4} x+z_{3}^{6}$ & 5 & 4 & 32 & 28 & 9 & 0 & $\mathbb{Z}_{2}$ \\
\hline$(9,9)$ & $E_{6}$ & 2 & 6 & $x^{3}+t^{4}+z_{2}^{2} t+z_{3}^{6} t+z_{3}^{8}$ & 3 & 0 & 35 & 35 & 3 & 2 & $\mathbb{Z}_{3}^{2}$ \\
& $E_{6}$ & 3 & 3 & $x^{3}+t^{4}+z_{2}^{3} t+z_{3}^{3} t+z_{3}^{4}$ & 3 & 0 & 27 & 27 & 3 & 4 & $\mathbb{Z}_{3}^{3}$ \\
\hline
\end{tabular}

Table 17. Sporadic models for $G=E_{6}$. Note that there are no solutions with $\left(b_{2}, b_{3}\right)=(8,9)$, and the two solutions for $\left(b_{2}, b_{3}\right)=(12,8)$ are the same.

\begin{tabular}{|c|c|cc|c|ccc|cc|cc|}
\hline$\left(b_{2}, b_{3}\right)$ & $G$ & $p_{2}$ & $p_{3}$ & $F$ & $r$ & $f$ & $d_{\mathrm{H}}$ & $\widehat{r}$ & $\widehat{d}_{\mathrm{H}}$ & $b_{3}$ & $\mathfrak{f}$ \\
\hline$(18,14)$ & $E_{7}$ & 9 & 2 & $x^{3}+x t^{3}+z_{2}^{9}+z_{3}^{2} t$ & 6 & 2 & 45 & 43 & 8 & 6 & $\mathbb{Z}_{2}^{3}$ \\
\hline$(14,14)$ & $E_{7}$ & 2 & 7 & $x^{3}+x t^{3}+z_{2}^{2} t+z_{3}^{7} t+z_{3}^{9}$ & 6 & 2 & 45 & 43 & 8 & 6 & $\mathbb{Z}_{2}^{3}$ \\
\hline
\end{tabular}

Table 18. Sporadic models for $G=E_{7}$. The two theories are equivalent.

\begin{tabular}{|c|c|cc|c|ccc|cc|cc|}
\hline$\left(b_{2}, b_{3}\right)$ & $G$ & $p_{2}$ & $p_{3}$ & $F$ & $r$ & $f$ & $d_{\mathrm{H}}$ & $\widehat{r}$ & $\widehat{d}_{\mathrm{H}}$ & $b_{3}$ & $\mathfrak{f}$ \\
\hline$(30,24)$ & $E_{8}$ & 10 & 2 & $x^{3}+t^{5}+z_{2}^{10}+z_{3}^{2} t$ & 10 & 0 & 54 & 54 & 10 & 8 & $\mathbb{Z}_{3}^{2}$ \\
\hline$(30,20)$ & $E_{8}$ & 3 & 4 & $x^{3}+t^{5}+z_{2}^{3}+z_{3}^{4} x$ & 12 & 0 & 40 & 40 & 12 & 8 & $\mathbb{Z}_{5}$ \\
& $E_{8}$ & 6 & 2 & $x^{3}+t^{5}+z_{2}^{6}+z_{3}^{2} x$ & 12 & 0 & 40 & 40 & 12 & 8 & $\mathbb{Z}_{5}$ \\
\hline$(24,24)$ & $E_{8}$ & 2 & 8 & $x^{3}+t^{5}+z_{2}^{2} t+z_{3}^{8} t+z_{3}^{10}$ & 10 & 0 & 54 & 54 & 10 & 4 & $\mathbb{Z}_{3}^{2}$ \\
\hline$(20,20)$ & $E_{8}$ & 2 & 4 & $x^{3}+t^{5}+z_{2}^{2} x+z_{3}^{4} x+z_{3}^{6}$ & 12 & 0 & 40 & 40 & 12 & 0 & $\mathbb{Z}_{5}$ \\
\hline
\end{tabular}

Table 19. Sporadic models with $G=E_{8}$. Note that there are no solutions with $\left(b_{2}, b_{3}\right)=(24,20)$. The two cases for $\left(b_{2}, b_{3}\right)=(30,20)$ are equivalent. 


\begin{tabular}{|c|c|c|c|c|c|c|c|c|c|c|c|}
\hline$\left(b_{2}, b_{3}\right)$ & $G$ & $p_{2}$ & $p_{3}$ & $F$ & $r$ & $f$ & $d_{\mathrm{H}}$ & $\widehat{r}$ & $\widehat{d}_{\mathrm{H}}$ & $b_{3}$ & $\mathfrak{f}$ \\
\hline \multirow[t]{12}{*}{$(2 N-2,2 N-2)$} & $\mathrm{SO}(8)$ & 4 & 12 & $x^{3}+y^{2}+z_{2}^{4}+z_{3}^{12}$ & 0 & 6 & 36 & 30 & 6 & 0 & 0 \\
\hline & $\mathrm{SO}(8)$ & 6 & 6 & $x^{3}+y^{2}+z_{2}^{6}+z_{3}^{6}$ & 1 & 8 & 29 & 21 & 9 & 0 & 0 \\
\hline & $\mathrm{SO}(10)$ & 3 & 24 & $x^{4}+y^{2}+z_{2}^{3}+z_{3}^{24}$ & 0 & 6 & 72 & 66 & 6 & 0 & 0 \\
\hline & $\mathrm{SO}(10)$ & 4 & 8 & $x^{4}+y^{2}+z_{2}^{4}+z_{3}^{8}$ & 2 & 7 & 35 & 28 & 9 & 2 & $\mathbb{Z}_{2}$ \\
\hline & $\mathrm{SO}(12)$ & 3 & 15 & $x^{5}+y^{2}+z_{2}^{3}+z_{3}^{15}$ & 0 & 0 & 56 & 56 & 0 & 0 & $\mathbb{Z}_{2}^{4}$ \\
\hline & $\mathrm{SO}(12)$ & 5 & 5 & $x^{5}+y^{2}+z_{2}^{5}+z_{3}^{5}$ & 2 & 0 & 32 & 32 & 2 & 12 & $\mathbb{Z}_{2}^{6}$ \\
\hline & $\mathrm{SO}(14)$ & 3 & 12 & $x^{6}+y^{2}+z_{2}^{3}+z_{3}^{12}$ & 2 & 8 & 59 & 51 & 10 & 2 & $\mathbb{Z}_{2}$ \\
\hline & $\mathrm{SO}(14)$ & 4 & 6 & $x^{6}+y^{2}+z_{2}^{4}+z_{3}^{6}$ & 4 & 5 & 40 & 35 & 9 & 4 & $\mathbb{Z}_{2}^{2}$ \\
\hline & $\mathrm{SO}(20)$ & 3 & 9 & $x^{9}+y^{2}+z_{2}^{3}+z_{3}^{9}$ & 3 & 0 & 64 & 64 & 3 & 14 & $\mathbb{Z}_{2}^{7}$ \\
\hline & $\mathrm{SO}(22)$ & 4 & 5 & $x^{10}+y^{2}+z_{2}^{4}+z_{3}^{5}$ & 6 & 4 & 56 & 52 & 10 & 4 & $\mathbb{Z}_{2}^{2}$ \\
\hline & $\mathrm{SO}(26)$ & 3 & 8 & $x^{12}+y^{2}+z_{2}^{3}+z_{3}^{8}$ & 5 & 6 & 80 & 74 & 11 & 2 & $\mathbb{Z}_{2}$ \\
\hline & $\mathrm{SO}(44)$ & 3 & 7 & $x^{21}+y^{2}+z_{2}^{3}+z_{3}^{7}$ & 6 & 0 & 120 & 120 & 6 & 12 & $\mathbb{Z}_{2}^{6}$ \\
\hline \multirow[t]{21}{*}{$(2 N-2, N)$} & $\mathrm{SO}(8)$ & 4 & 8 & $x^{3}+y^{2}+z_{2}^{4}+z_{3}^{12}$ & 0 & 6 & 36 & 30 & 6 & 0 & 0 \\
\hline & $\mathrm{SO}(8)$ & 5 & 5 & $x^{3}+y^{2}+z_{2}^{5}+z_{3}^{5} x$ & 0 & 0 & 26 & 26 & 0 & 0 & $\mathbb{Z}_{2}^{2}$ \\
\hline & $\mathrm{SO}(8)$ & 6 & 4 & $x^{3}+y^{2}+z_{2}^{6}+z_{3}^{6}$ & 1 & 8 & 29 & 21 & 9 & 0 & 0 \\
\hline & $\mathrm{SO}(8)$ & 9 & 3 & $x^{3}+y^{2}+z_{2}^{9}+z_{3}^{3} x$ & 0 & 0 & 28 & 28 & 0 & 0 & $\mathbb{Z}_{2}^{3}$ \\
\hline & $\mathrm{SO}(10)$ & 3 & 15 & $x^{4}+y^{2}+z_{2}^{3}+z_{3}^{24}$ & 0 & 6 & 72 & 66 & 6 & 0 & 0 \\
\hline & $\mathrm{SO}(10)$ & 4 & 5 & $x^{4}+y^{2}+z_{2}^{4}+z_{3}^{8}$ & 2 & 7 & 35 & 28 & 9 & 2 & $\mathbb{Z}_{2}$ \\
\hline & $\mathrm{SO}(10)$ & 6 & 3 & $x^{4}+y^{2}+z_{2}^{6}+z_{3}^{4} z_{2}$ & 2 & 3 & 30 & 27 & 5 & 0 & 0 \\
\hline & $\mathrm{SO}(10)$ & 16 & 2 & $x^{4}+y^{2}+z_{2}^{16}+z_{3}^{3} z_{2}$ & 1 & 7 & 53 & 46 & 8 & 0 & 0 \\
\hline & $\mathrm{SO}(12)$ & 3 & 9 & $x^{5}+y^{2}+z_{2}^{3}+z_{3}^{15}$ & 0 & 0 & 56 & 56 & 0 & 0 & $\mathbb{Z}_{2}^{4}$ \\
\hline & $\mathrm{SO}(12)$ & 4 & 4 & $x^{5}+y^{2}+z_{2}^{4}+z_{3}^{5} z_{2}$ & 2 & 4 & 36 & 32 & 6 & 0 & 0 \\
\hline & $\mathrm{SO}(12)$ & 5 & 3 & $x^{5}+y^{2}+z_{2}^{5}+z_{3}^{5}$ & 2 & 0 & 32 & 32 & 2 & 12 & $\mathbb{Z}_{2}^{6}$ \\
\hline & $\mathrm{SO}(12)$ & 10 & 2 & $x^{5}+y^{2}+z_{2}^{10}+z_{3}^{3} z_{2}$ & 2 & 8 & 46 & 38 & 10 & 0 & 0 \\
\hline & $\mathrm{SO}(14)$ & 3 & 7 & $x^{6}+y^{2}+z_{2}^{3}+z_{3}^{12}$ & 2 & 8 & 59 & 51 & 10 & 2 & $\mathbb{Z}_{2}$ \\
\hline & $\mathrm{SO}(14)$ & 8 & 2 & $x^{6}+y^{2}+z_{2}^{8}+z_{3}^{3} z_{2}$ & 3 & 5 & 45 & 40 & 8 & 0 & 0 \\
\hline & $\mathrm{SO}(16)$ & 3 & 6 & $x^{7}+y^{2}+z_{2}^{3}+z_{3}^{9} x$ & 1 & 0 & 57 & 57 & 1 & 0 & $\mathbb{Z}_{2}^{3}$ \\
\hline & $\mathrm{SO}(16)$ & 7 & 2 & $x^{7}+y^{2}+z_{2}^{7}+z_{3}^{3} x$ & 3 & 0 & 45 & 45 & 3 & 12 & $\mathbb{Z}_{2}^{6}$ \\
\hline & $\mathrm{SO}(18)$ & 4 & 3 & $x^{8}+y^{2}+z_{2}^{4}+z_{3}^{4} z_{2}$ & 5 & 5 & 48 & 43 & 10 & 2 & $\mathbb{Z}_{2}$ \\
\hline & $\mathrm{SO}(20)$ & 3 & 5 & $x^{9}+y^{2}+z_{2}^{3}+z_{3}^{9}$ & 3 & 0 & 64 & 64 & 3 & 14 & $\mathbb{Z}_{2}^{7}$ \\
\hline & $\mathrm{SO}(20)$ & 6 & 2 & $x^{9}+y^{2}+z_{2}^{6}+z_{3}^{3} z_{2}$ & 5 & 6 & 55 & 49 & 11 & 0 & 0 \\
\hline & $\mathrm{SO}(32)$ & 3 & 4 & $x^{15}+y^{2}+z_{2}^{3}+z_{3}^{4} z_{2}$ & 2 & 4 & 72 & 68 & 6 & 4 & $\mathbb{Z}_{5}$ \\
\hline & $\mathrm{SO}(32)$ & 5 & 2 & $x^{15}+y^{2}+z_{2}^{5}+z_{3}^{3} z_{2}$ & 6 & 0 & 77 & 77 & 6 & 10 & $\mathbb{Z}_{2}^{5}$ \\
\hline \multirow[t]{7}{*}{$(N, N)$} & $\mathrm{SO}(8)$ & 3 & 6 & $x^{3}+y^{2}+z_{2}^{4} z_{3}+z_{3}^{9}$ & 0 & 0 & 28 & 28 & 0 & 0 & $\mathbb{Z}_{2}^{3}$ \\
\hline & $\mathrm{SO}(8)$ & 4 & 4 & $x^{3}+y^{2}+z_{2}^{6}+z_{3}^{6}$ & 1 & 8 & 29 & 21 & 9 & 0 & 0 \\
\hline & $\mathrm{SO}(10)$ & 2 & 10 & $x^{4}+y^{2}+z_{2}^{3} z_{3}+z_{3}^{16}$ & 1 & 7 & 53 & 46 & 8 & 0 & 0 \\
\hline & $\mathrm{SO}(12)$ & 2 & 6 & $x^{5}+y^{2}+z_{2}^{3} z_{3}+z_{3}^{10}$ & 2 & 8 & 46 & 38 & 10 & 0 & 0 \\
\hline & $\mathrm{SO}(12)$ & 3 & 3 & $x^{5}+y^{2}+z_{2}^{5}+z_{3}^{5}$ & 2 & 0 & 32 & 32 & 2 & 12 & $\mathbb{Z}_{2}^{6}$ \\
\hline & $\mathrm{SO}(16)$ & 2 & 4 & $x^{7}+y^{2}+z_{2}^{3} z_{3}+z_{3}^{7}$ & 3 & 0 & 45 & 45 & 3 & 12 & $\mathbb{Z}_{2}^{6}$ \\
\hline & $\mathrm{SO}(24)$ & 2 & 3 & $x^{11}+y^{2}+z_{2}^{3} z_{3}+z_{3}^{4} z_{2}$ & 5 & 0 & 60 & 60 & 5 & 10 & $\mathbb{Z}_{2}^{5}$ \\
\hline
\end{tabular}

Table 20. $G=\mathrm{SO}(2 N)$ models with $b_{1}=N, p_{1}=1$. 


\begin{tabular}{|c|c|c|c|c|c|c|c|c|c|c|}
\hline$\left(b_{1}, b_{2}, b_{3}\right)$ & $p_{2}$ & $p_{3}$ & $F$ & $r$ & $f$ & $d_{\mathrm{H}}$ & $\widehat{r}$ & $\widehat{d}_{\mathrm{H}}$ & $b_{3}$ & $\mathfrak{f}$ \\
\hline \multirow[t]{3}{*}{$(9,12,12)$} & 5 & 20 & $x^{3}+y^{2}+z_{2}^{5}+z_{3}^{20}$ & 0 & 0 & 76 & 76 & 0 & 0 & $\mathbb{Z}_{3}^{2}$ \\
\hline & 6 & 12 & $x^{3}+y^{2}+z_{2}^{6}+z_{3}^{12}$ & 2 & 8 & 59 & 51 & 10 & 2 & $\mathbb{Z}_{2}$ \\
\hline & 8 & 8 & $x^{3}+y^{2}+z_{2}^{8}+z_{3}^{8}$ & 2 & 0 & 49 & 49 & 2 & 6 & $\mathbb{Z}_{3}^{3}$ \\
\hline \multirow[t]{6}{*}{$(9,12,9)$} & 5 & 15 & $x^{3}+y^{2}+z_{2}^{5}+z_{3}^{20}$ & 0 & 0 & 76 & 76 & 0 & 0 & $\mathbb{Z}_{3}^{2}$ \\
\hline & 6 & 9 & $x^{3}+y^{2}+z_{2}^{6}+z_{3}^{12}$ & 2 & 8 & 59 & 51 & 10 & 2 & $\mathbb{Z}_{2}$ \\
\hline & 7 & 7 & $x^{3}+y^{2}+z_{2}^{7}+z_{3}^{8} z_{2}$ & 1 & 0 & 50 & 50 & 1 & 0 & $\mathbb{Z}_{3}$ \\
\hline & 8 & 6 & $x^{3}+y^{2}+z_{2}^{8}+z_{3}^{8}$ & 2 & 0 & 49 & 49 & 2 & 6 & $\mathbb{Z}_{3}^{3}$ \\
\hline & 10 & 5 & $x^{3}+y^{2}+z_{2}^{10}+z_{3}^{6} z_{2}$ & 1 & 0 & 51 & 51 & 1 & 0 & $\mathbb{Z}_{3}$ \\
\hline & 16 & 4 & $x^{3}+y^{2}+z_{2}^{16}+z_{3}^{5} z_{2}$ & 1 & 0 & 65 & 65 & 1 & 0 & $\mathbb{Z}_{3}^{2}$ \\
\hline \multirow[t]{3}{*}{$(9,12,8)$} & 6 & 8 & $x^{3}+y^{2}+z_{2}^{6}+z_{3}^{8} x$ & 2 & 8 & 59 & 51 & 10 & 2 & $\mathbb{Z}_{2}$ \\
\hline & 12 & 4 & $x^{3}+y^{2}+z_{2}^{12}+z_{3}^{4} x$ & 2 & 8 & 59 & 51 & 10 & 2 & $\mathbb{Z}_{2}$ \\
\hline & 36 & 3 & $x^{3}+y^{2}+z_{2}^{36}+z_{3}^{3} x$ & 0 & 7 & 126 & 119 & 7 & 0 & 0 \\
\hline \multirow[t]{2}{*}{$(9,9,9)$} & 4 & 12 & $x^{3}+y^{2}+z_{2}^{5} z_{3}+z_{3}^{16}$ & 1 & 0 & 65 & 65 & 1 & 0 & $\mathbb{Z}_{3}^{2}$ \\
\hline & 6 & 6 & $x^{3}+y^{2}+z_{2}^{8}+z_{3}^{8}$ & 2 & 0 & 49 & 49 & 2 & 6 & $\mathbb{Z}_{3}^{3}$ \\
\hline \multirow[t]{2}{*}{$(9,9,8)$} & 9 & 4 & $x^{3}+y^{2}+z_{2}^{12}+z_{3}^{4} x$ & 2 & 8 & 59 & 51 & 10 & 2 & $\mathbb{Z}_{2}$ \\
\hline & 27 & 3 & $x^{3}+y^{2}+z_{2}^{36}+z_{3}^{3} x$ & 0 & 7 & 126 & 119 & 7 & 0 & 0 \\
\hline \multirow[t]{2}{*}{$(9,8,8)$} & 3 & 24 & $x^{3}+y^{2}+z_{2}^{3} x+z_{3}^{24} x+z_{3}^{36}$ & 0 & 7 & 126 & 119 & 7 & 0 & 0 \\
\hline & 4 & 8 & $x^{3}+y^{2}+z_{2}^{4} x+z_{3}^{8} x+z_{3}^{12}$ & 2 & 8 & 59 & 51 & 10 & 2 & $\mathbb{Z}_{2}$ \\
\hline \multirow[t]{2}{*}{$(8,12,12)$} & 4 & 12 & $t^{4}+y^{2}+z_{2}^{4}+z_{3}^{12}$ & 3 & 7 & 53 & 46 & 10 & 4 & $\mathbb{Z}_{3}$ \\
\hline & 6 & 6 & $t^{4}+y^{2}+z_{2}^{6}+z_{3}^{6}$ & 4 & 5 & 40 & 35 & 9 & 4 & $\mathbb{Z}_{2}^{2}$ \\
\hline \multirow[t]{2}{*}{$(8,12,9)$} & 4 & 9 & $t^{4}+y^{2}+z_{2}^{4}+z_{3}^{9} t$ & 3 & 7 & 53 & 46 & 10 & 4 & $\mathbb{Z}_{3}$ \\
\hline & 12 & 3 & $t^{4}+y^{2}+z_{2}^{12}+z_{3}^{3} t$ & 3 & 7 & 53 & 46 & 10 & 4 & $\mathbb{Z}_{3}$ \\
\hline \multirow[t]{4}{*}{$(8,12,8)$} & 4 & 8 & $t^{4}+y^{2}+z_{2}^{4}+z_{3}^{12}$ & 3 & 7 & 53 & 46 & 10 & 4 & $\mathbb{Z}_{3}$ \\
\hline & 5 & 5 & $t^{4}+y^{2}+z_{2}^{5}+z_{3}^{6} z_{2}$ & 3 & 2 & 40 & 38 & 5 & 0 & 0 \\
\hline & 6 & 4 & $t^{4}+y^{2}+z_{2}^{6}+z_{3}^{6}$ & 4 & 5 & 40 & 35 & 9 & 4 & $\mathbb{Z}_{2}^{2}$ \\
\hline & 9 & 3 & $t^{4}+y^{2}+z_{2}^{9}+z_{3}^{4} z_{2}$ & 4 & 4 & 44 & 40 & 8 & 0 & 0 \\
\hline$(8,9,9)$ & 3 & 9 & $t^{4}+y^{2}+z_{2}^{3} t+z_{3}^{9} t+z_{3}^{12}$ & 3 & 7 & 53 & 46 & 10 & 4 & $\mathbb{Z}_{3}$ \\
\hline$(8,9,8)$ & 3 & 8 & $t^{4}+y^{2}+z_{2}^{3} t+z_{3}^{12}$ & 3 & 7 & 53 & 46 & 10 & 4 & $\mathbb{Z}_{3}$ \\
\hline \multirow[t]{2}{*}{$(8,8,8)$} & 3 & 6 & $t^{4}+y^{2}+z_{2}^{4} z_{3}+z_{3}^{9}$ & 4 & 4 & 44 & 40 & 8 & 0 & 0 \\
\hline & 4 & 4 & $t^{4}+y^{2}+z_{2}^{6}+z_{3}^{6}$ & 4 & 5 & 40 & 35 & 9 & 4 & $\mathbb{Z}_{2}^{2}$ \\
\hline
\end{tabular}

Table 21. Models for $G=E_{6}$ with $b_{1}=8,9$. 


\begin{tabular}{|c|cc|c|ccc|cc|cc|}
\hline$\left(b_{2}, b_{3}\right)$ & $p_{2}$ & $p_{3}$ & $F$ & $r$ & $f$ & $d_{\mathrm{H}}$ & $\widehat{r}$ & $\widehat{d}_{\mathrm{H}}$ & $b_{3}$ & $\mathfrak{f}$ \\
\hline$(18,18)$ & 5 & 45 & $x^{3}+y^{2}+z_{2}^{5}+z_{3}^{45}$ & 0 & 0 & 176 & 176 & 0 & 0 & $\mathbb{Z}_{2}^{4}$ \\
& 6 & 18 & $x^{3}+y^{2}+z_{2}^{6}+z_{3}^{18}$ & 3 & 8 & 89 & 81 & 11 & 4 & $\mathbb{Z}_{3}$ \\
& 9 & 9 & $x^{3}+y^{2}+z_{2}^{9}+z_{3}^{9}$ & 3 & 0 & 64 & 64 & 3 & 14 & $\mathbb{Z}_{2}^{7}$ \\
\hline$(18,14)$ & 5 & 35 & $x^{3}+y^{2}+z_{2}^{5}+z_{3}^{45}$ & 0 & 0 & 176 & 176 & 0 & 0 & $\mathbb{Z}_{2}^{4}$ \\
& 6 & 14 & $x^{3}+y^{2}+z_{2}^{6}+z_{3}^{18}$ & 3 & 8 & 89 & 81 & 11 & 4 & $\mathbb{Z}_{3}$ \\
& 8 & 8 & $x^{3}+y^{2}+z_{2}^{8}+z_{3}^{9} z_{2}$ & 3 & 2 & 66 & 64 & 5 & 0 & 0 \\
& 9 & 7 & $x^{3}+y^{2}+z_{2}^{9}+z_{3}^{9}$ & 3 & 0 & 64 & 64 & 3 & 14 & $\mathbb{Z}_{2}^{7}$ \\
& 15 & 5 & $x^{3}+y^{2}+z_{2}^{15}+z_{3}^{6} z_{2}$ & 2 & 0 & 76 & 76 & 2 & 0 & $\mathbb{Z}_{2}$ \\
& 36 & 4 & $x^{3}+y^{2}+z_{2}^{36}+z_{3}^{5} z_{2}$ & 1 & 8 & 149 & 141 & 9 & 0 & 0 \\
\hline$(14,14)$ & 4 & 28 & $x^{3}+y^{2}+z_{2}^{5} z_{3}+z_{3}^{36}$ & 1 & 8 & 149 & 141 & 9 & 0 & 0 \\
& 7 & 7 & $x^{3}+y^{2}+z_{2}^{9}+z_{3}^{9}$ & 3 & 0 & 64 & 64 & 3 & 14 & $\mathbb{Z}_{2}^{7}$ \\
\hline
\end{tabular}

Table 22. $G=E_{7}$ with $b_{1}=14$ models. 


\begin{tabular}{|c|c|c|c|c|c|c|c|c|c|c|}
\hline$\left(b_{1}, b_{2}, b_{3}\right)$ & $p_{2}$ & $p_{3}$ & $F$ & $r$ & $f$ & $d_{\mathrm{H}}$ & $\widehat{r}$ & $\widehat{d}_{\mathrm{H}}$ & $b_{3}$ & $\mathfrak{f}$ \\
\hline \multirow[t]{2}{*}{$(24,30,30)$} & 6 & 30 & $x^{3}+y^{2}+z_{2}^{6}+z_{3}^{30}$ & 5 & 8 & 149 & 141 & 13 & 8 & $\mathbb{Z}_{5}$ \\
\hline & 10 & 10 & $x^{3}+y^{2}+z_{2}^{10}+z_{3}^{10}$ & 5 & 0 & 81 & 81 & 5 & 16 & $\mathbb{Z}_{3}^{4}$ \\
\hline \multirow[t]{6}{*}{$(24,30,24)$} & 6 & 24 & $x^{3}+y^{2}+z_{2}^{6}+z_{3}^{30}$ & 5 & 8 & 149 & 141 & 13 & 8 & $\mathbb{Z}_{5}$ \\
\hline & 7 & 14 & $x^{3}+y^{2}+z_{2}^{7}+z_{3}^{15} z_{2}$ & 4 & 0 & 99 & 99 & 4 & 0 & 0 \\
\hline & 9 & 9 & $x^{3}+y^{2}+z_{2}^{9}+z_{3}^{10} z_{2}$ & 6 & 0 & 82 & 82 & 6 & 0 & $\mathbb{Z}_{2}$ \\
\hline & 10 & 8 & $x^{3}+y^{2}+z_{2}^{10}+z_{3}^{10}$ & 5 & 0 & 81 & 81 & 5 & 16 & $\mathbb{Z}_{3}^{4}$ \\
\hline & 15 & 6 & $x^{3}+y^{2}+z_{2}^{15}+z_{3}^{7} z_{2}$ & 5 & 0 & 91 & 91 & 5 & 12 & $\mathbb{Z}_{2}^{6}$ \\
\hline & 25 & 5 & $x^{3}+y^{2}+z_{2}^{25}+z_{3}^{6} z_{2}$ & 4 & 0 & 126 & 126 & 4 & 0 & 0 \\
\hline \multirow[t]{3}{*}{$(24,30,20)$} & 6 & 20 & $x^{3}+y^{2}+z_{2}^{6}+z_{3}^{20} x$ & 5 & 8 & 149 & 141 & 13 & 8 & $\mathbb{Z}_{5}$ \\
\hline & 15 & 5 & $x^{3}+y^{2}+z_{2}^{15}+z_{3}^{5} x$ & 5 & 0 & 91 & 91 & 5 & 12 & $\mathbb{Z}_{2}^{6}$ \\
\hline & 30 & 4 & $x^{3}+y^{2}+z_{2}^{30}+z_{3}^{4} x$ & 5 & 8 & 149 & 141 & 13 & 8 & $\mathbb{Z}_{5}$ \\
\hline \multirow[t]{3}{*}{$(24,24,24)$} & 5 & 20 & $x^{3}+y^{2}+z_{2}^{6} z_{3}+z_{3}^{25}$ & 4 & 0 & 126 & 126 & 4 & 0 & 0 \\
\hline & 6 & 12 & $x^{3}+y^{2}+z_{2}^{7} z_{3}+z_{3}^{15}$ & 5 & 0 & 91 & 91 & 5 & 12 & $\mathbb{Z}_{2}^{6}$ \\
\hline & 8 & 8 & $x^{3}+y^{2}+z_{2}^{10}+z_{3}^{10}$ & 5 & 0 & 81 & 81 & 5 & 16 & $\mathbb{Z}_{3}^{4}$ \\
\hline \multirow[t]{4}{*}{$(24,24,20)$} & 6 & 10 & $x^{3}+y^{2}+z_{2}^{7} z_{3}+z_{3}^{10} x$ & 5 & 0 & 91 & 91 & 5 & 12 & $\mathbb{Z}_{2}^{6}$ \\
\hline & 9 & 6 & $x^{3}+y^{2}+z_{2}^{10} z_{3}+z_{3}^{6} x$ & 6 & 0 & 82 & 82 & 6 & 0 & $\mathbb{Z}_{2}$ \\
\hline & 12 & 5 & $x^{3}+y^{2}+z_{2}^{15}+z_{3}^{5} x$ & 5 & 0 & 91 & 91 & 5 & 12 & $\mathbb{Z}_{2}^{6}$ \\
\hline & 24 & 4 & $x^{3}+y^{2}+z_{2}^{30}+z_{3}^{4} x$ & 5 & 8 & 149 & 141 & 13 & 8 & $\mathbb{Z}_{5}$ \\
\hline \multirow[t]{2}{*}{$(24,20,20)$} & 4 & 20 & $x^{3}+y^{2}+z_{2}^{4} x+z_{3}^{20} x+z_{3}^{30}$ & 5 & 8 & 149 & 141 & 13 & 8 & $\mathbb{Z}_{5}$ \\
\hline & 5 & 10 & $x^{3}+y^{2}+z_{2}^{5} x+z_{3}^{10} x+z_{3}^{15}$ & 5 & 0 & 91 & 91 & 5 & 4 & $\mathbb{Z}_{2}^{6}$ \\
\hline \multirow[t]{2}{*}{$(20,30,30)$} & 4 & 12 & $t^{5}+y^{2}+z_{2}^{4}+z_{3}^{12}$ & 8 & 0 & 66 & 66 & 8 & 4 & $\mathbb{Z}_{5}$ \\
\hline & 6 & 6 & $t^{5}+y^{2}+z_{2}^{6}+z_{3}^{6}$ & 6 & 0 & 50 & 50 & 6 & 16 & $\mathbb{Z}_{5}^{2}$ \\
\hline \multirow[t]{2}{*}{$(20,30,24)$} & 5 & 6 & $t^{5}+y^{2}+z_{2}^{5}+z_{3}^{6} t$ & 10 & 0 & 52 & 52 & 10 & 4 & $\mathbb{Z}_{2}^{2}$ \\
\hline & 15 & 3 & $t^{5}+y^{2}+z_{2}^{15}+z_{3}^{3} t$ & 6 & 0 & 77 & 77 & 6 & 10 & $\mathbb{Z}_{2}^{5}$ \\
\hline \multirow[t]{4}{*}{$(20,30,20)$} & 4 & 8 & $t^{5}+y^{2}+z_{2}^{4}+z_{3}^{12}$ & 8 & 0 & 66 & 66 & 8 & 4 & $\mathbb{Z}_{5}$ \\
\hline & 5 & 5 & $t^{5}+y^{2}+z_{2}^{5}+z_{3}^{6} z_{2}$ & 10 & 0 & 52 & 52 & 10 & 4 & $\mathbb{Z}_{2}^{2}$ \\
\hline & 6 & 4 & $t^{5}+y^{2}+z_{2}^{6}+z_{3}^{6}$ & 6 & 0 & 50 & 50 & 6 & 16 & $\mathbb{Z}_{5}^{2}$ \\
\hline & 9 & 3 & $t^{5}+y^{2}+z_{2}^{9}+z_{3}^{4} z_{2}$ & 8 & 0 & 56 & 56 & 8 & 0 & 0 \\
\hline \multirow[t]{2}{*}{$(20,24,24)$} & 3 & 12 & $t^{5}+y^{2}+z_{2}^{3} t+z_{3}^{12} t+z_{3}^{15}$ & 6 & 0 & 77 & 77 & 6 & 10 & $\mathbb{Z}_{2}^{5}$ \\
\hline & 4 & 6 & $t^{5}+y^{2}+z_{2}^{4} t+z_{3}^{6} t+z_{2}^{5}$ & 10 & 0 & 52 & 52 & 10 & 4 & $\mathbb{Z}_{2}^{2}$ \\
\hline \multirow[t]{2}{*}{$(20,24,20)$} & 3 & 10 & $t^{5}+y^{2}+z_{2}^{3} t+z_{3}^{15}$ & 6 & 0 & 77 & 77 & 6 & 10 & $\mathbb{Z}_{2}^{5}$ \\
\hline & 4 & 5 & $t^{5}+y^{2}+z_{2}^{4} t+z_{3}^{6} t+z_{2}^{5}$ & 10 & 0 & 52 & 52 & 10 & 4 & $\mathbb{Z}_{2}^{2}$ \\
\hline \multirow[t]{2}{*}{$(20,20,20)$} & 3 & 6 & $t^{5}+y^{2}+z_{2}^{4} z_{3}+z_{3}^{9}$ & 8 & 0 & 56 & 56 & 8 & 0 & 0 \\
\hline & 4 & 4 & $t^{5}+y^{2}+z_{2}^{6}+z_{3}^{6}$ & 6 & 0 & 50 & 50 & 6 & 16 & $\mathbb{Z}_{5}^{2}$ \\
\hline
\end{tabular}

Table 23. $G=E_{8}$ models with $b_{1}=20,24$. 
Open Access. This article is distributed under the terms of the Creative Commons Attribution License (CC-BY 4.0), which permits any use, distribution and reproduction in any medium, provided the original author(s) and source are credited.

\section{References}

[1] E. Witten, Phase transitions in M-theory and F-theory, Nucl. Phys. B 471 (1996) 195 [hep-th/9603150] [INSPIRE].

[2] D.R. Morrison and N. Seiberg, Extremal transitions and five-dimensional supersymmetric field theories, Nucl. Phys. B 483 (1997) 229 [hep-th/9609070] [INSPIRE].

[3] M.R. Douglas, S.H. Katz and C. Vafa, Small instantons, del Pezzo surfaces and type-I-prime theory, Nucl. Phys. B 497 (1997) 155 [hep-th/9609071] [INSPIRE].

[4] K.A. Intriligator, D.R. Morrison and N. Seiberg, Five-dimensional supersymmetric gauge theories and degenerations of Calabi-Yau spaces, Nucl. Phys. B 497 (1997) 56 [hep-th/9702198] [INSPIRE].

[5] H. Hayashi, C. Lawrie and S. Schäfer-Nameki, Phases, Flops and F-theory: SU(5) Gauge Theories, JHEP 10 (2013) 046 [arXiv:1304.1678] [InSPIRE].

[6] H. Hayashi, C. Lawrie, D.R. Morrison and S. Schäfer-Nameki, Box Graphs and Singular Fibers, JHEP 05 (2014) 048 [arXiv: 1402.2653] [INSPIRE].

[7] M. Del Zotto, J.J. Heckman and D.R. Morrison, 6D SCFTs and Phases of 5D Theories, JHEP 09 (2017) 147 [arXiv: 1703.02981] [INSPIRE].

[8] D. Xie and S.-T. Yau, Three dimensional canonical singularity and five dimensional $\mathcal{N}=1$ SCFT, JHEP 06 (2017) 134 [arXiv:1704.00799] [INSPIRE].

[9] P. Jefferson, H.-C. Kim, C. Vafa and G. Zafrir, Towards Classification of 5d SCFTs: Single Gauge Node, arXiv:1705.05836 [INSPIRE].

[10] P. Jefferson, S. Katz, H.-C. Kim and C. Vafa, On Geometric Classification of 5d SCFTs, JHEP 04 (2018) 103 [arXiv: 1801.04036] [INSPIRE].

[11] L. Bhardwaj and P. Jefferson, Classifying 5d SCFTs via 6d SCFTs: Rank one, JHEP 07 (2019) 178 [Addendum JHEP 01 (2020) 153] [arXiv:1809.01650] [INSPIRE].

[12] L. Bhardwaj and P. Jefferson, Classifying 5d SCFTs via 6d SCFTs: Arbitrary rank, JHEP 10 (2019) 282 [arXiv:1811.10616] [INSPIRE].

[13] F. Apruzzi, L. Lin and C. Mayrhofer, Phases of $5 d$ SCFTs from M-/F-theory on Non-Flat Fibrations, JHEP 05 (2019) 187 [arXiv: 1811.12400] [INSPIRE].

[14] C. Closset, M. Del Zotto and V. Saxena, Five-dimensional SCFTs and gauge theory phases: an M-theory/type IIA perspective, SciPost Phys. 6 (2019) 052 [arXiv:1812.10451] [INSPIRE].

[15] F. Apruzzi, C. Lawrie, L. Lin, S. Schäfer-Nameki and Y.-N. Wang, 5d Superconformal Field Theories and Graphs, Phys. Lett. B 800 (2020) 135077 [arXiv: 1906.11820] [INSPIRE].

[16] F. Apruzzi, C. Lawrie, L. Lin, S. Schäfer-Nameki and Y.-N. Wang, Fibers add Flavor, Part I: Classification of 5d SCFTs, Flavor Symmetries and BPS States, JHEP 11 (2019) 068 [arXiv: 1907.05404$]$ [INSPIRE]. 
[17] F. Apruzzi, C. Lawrie, L. Lin, S. Schäfer-Nameki and Y.-N. Wang, Fibers add Flavor, Part II: 5d SCFTs, Gauge Theories, and Dualities, JHEP 03 (2020) 052 [arXiv: 1909.09128] [INSPIRE].

[18] L. Bhardwaj, On the classification of 5d SCFTs, JHEP 09 (2020) 007 [arXiv:1909.09635] [INSPIRE].

[19] L. Bhardwaj, P. Jefferson, H.-C. Kim, H.-C. Tarazi and C. Vafa, Twisted Circle Compactifications of 6d SCFTs, JHEP 12 (2020) 151 [arXiv:1909.11666] [INSPIRE].

[20] L. Bhardwaj, Dualities of 5d gauge theories from S-duality, JHEP 07 (2020) 012 [arXiv: 1909.05250] [INSPIRE].

[21] V. Saxena, Rank-two 5d SCFTs from M-theory at isolated toric singularities: a systematic study, JHEP 04 (2020) 198 [arXiv: 1911.09574] [INSPIRE].

[22] F. Apruzzi, S. Schäfer-Nameki and Y.-N. Wang, 5d SCFTs from Decoupling and Gluing, JHEP 08 (2020) 153 [arXiv: 1912.04264] [INSPIRE].

[23] C. Closset and M. Del Zotto, On 5d SCFTs and their BPS quivers. Part I: B-branes and brane tilings, arXiv:1912.13502 [INSPIRE].

[24] L. Bhardwaj, Do all 5d SCFTs descend from 6d SCFTs?, JHEP 04 (2021) 085 [arXiv: 1912.00025] [INSPIRE].

[25] L. Bhardwaj and G. Zafrir, Classification of $5 d \mathcal{N}=1$ gauge theories, JHEP 12 (2020) 099 [arXiv: 2003. 04333] [INSPIRE].

[26] J. Eckhard, S. Schäfer-Nameki and Y.-N. Wang, Trifectas for $T_{N}$ in 5d, JHEP 07 (2020) 199 [arXiv : 2004.15007] [INSPIRE].

[27] D.R. Morrison, S. Schäfer-Nameki and B. Willett, Higher-Form Symmetries in 5d, JHEP 09 (2020) 024 [arXiv: 2005.12296] [INSPIRE].

[28] F. Albertini, M. Del Zotto, I. García Etxebarria and S.S. Hosseini, Higher Form Symmetries and M-theory, JHEP 12 (2020) 203 [arXiv:2005.12831] [INSPIRE].

[29] L. Bhardwaj, More 5d KK theories, arXiv:2005.01722 [InSPIRE].

[30] M. van Beest, A. Bourget, J. Eckhard and S. Schäfer-Nameki, (Symplectic) Leaves and (5d Higgs) Branches in the Poly(go)nesian Tropical Rain Forest, JHEP 11 (2020) 124 [arXiv : 2008. 05577] [INSPIRE].

[31] M. Hübner, 5d SCFTs from $\left(E_{n}, E_{m}\right)$ conformal matter, JHEP 12 (2020) 014 [arXiv: 2006. 01694] [INSPIRE].

[32] L. Bhardwaj and S. Schäfer-Nameki, Higher-form symmetries of $6 d$ and $5 d$ theories, JHEP 02 (2021) 159 [arXiv:2008.09600] [INSPIRE].

[33] L. Bhardwaj, Flavor Symmetry of 5d SCFTs, Part 1: General Setup, arXiv:2010.13230 [INSPIRE].

[34] L. Bhardwaj, Flavor symmetry of 5d SCFTs. Part II. Applications, JHEP 04 (2021) 221 [arXiv: 2010.13235] [INSPIRE].

[35] M. Van Beest, A. Bourget, J. Eckhard and S. Schäfer-Nameki, (5d RG-flow) Trees in the Tropical Rain Forest, JHEP 03 (2021) 241 [arXiv:2011.07033] [INSPIRE].

[36] C. Closset, S. Schäfer-Nameki and Y.-N. Wang, Coulomb and Higgs Branches from Canonical Singularities: Part 0, JHEP 02 (2021) 003 [arXiv:2007.15600] [INSPIRE]. 
[37] C. Closset, S. Schäfer-Nameki and Y.-N. Wang, On SCFTs at canonical singularities: Part 1, in preparation.

[38] M. Caibar, Minimal models of canonical 3-fold singularities and their Betti numbers, Int. Math. Res. Not. 2005 (2005) 1563.

[39] P. Arras, A. Grassi and T. Weigand, Terminal Singularities, Milnor Numbers, and Matter in F-theory, J. Geom. Phys. 123 (2018) 71 [arXiv:1612.05646] [INSPIRE].

[40] A. Grassi and T. Weigand, On topological invariants of algebraic threefolds with (QQ-factorial) singularities, arXiv:1804.02424 [INSPIRE].

[41] G. Ferlito, A. Hanany, N. Mekareeya and G. Zafrir, $3 d$ Coulomb branch and $5 d$ Higgs branch at infinite coupling, JHEP 07 (2018) 061 [arXiv:1712.06604] [INSPIRE].

[42] S. Cabrera, A. Hanany and F. Yagi, Tropical Geometry and Five Dimensional Higgs Branches at Infinite Coupling, JHEP 01 (2019) 068 [arXiv:1810.01379] [INSPIRE].

[43] E. Witten, $\mathrm{SL}(2, \mathbb{Z})$ action on three-dimensional conformal field theories with Abelian symmetry, in proceedings of the From Fields to Strings: Circumnavigating Theoretical Physics: A Conference in Tribute to Ian Kogan, 8-10 January 2004, Oxford, U.K., hep-th/0307041 [INSPIRE].

[44] K. Hori, H. Ooguri and C. Vafa, NonAbelian conifold transitions and $N=4$ dualities in three-dimensions, Nucl. Phys. B 504 (1997) 147 [hep-th/9705220] [INSPIRE].

[45] M. Akhond, F. Carta, S. Dwivedi, H. Hayashi, S.-S. Kim and F. Yagi, Five-brane webs, Higgs branches and unitary/orthosymplectic magnetic quivers, JHEP 12 (2020) 164 [arXiv: 2008.01027] [INSPIRE].

[46] A. Bourget et al., The Higgs mechanism - Hasse diagrams for symplectic singularities, JHEP 01 (2020) 157 [arXiv: 1908.04245] [INSPIRE].

[47] A. Bourget, S. Cabrera, J.F. Grimminger, A. Hanany and Z. Zhong, Brane Webs and Magnetic Quivers for SQCD, JHEP 03 (2020) 176 [arXiv: 1909.00667] [INSPIRE].

[48] A. Bourget, J.F. Grimminger, A. Hanany, M. Sperling and Z. Zhong, Magnetic Quivers from Brane Webs with O5 Planes, JHEP 07 (2020) 204 [arXiv: 2004.04082] [INSPIRE].

[49] A. Bourget, J.F. Grimminger, A. Hanany, M. Sperling, G. Zafrir and Z. Zhong, Magnetic quivers for rank 1 theories, JHEP 09 (2020) 189 [arXiv:2006.16994] [INSPIRE].

[50] A. Bourget, J.F. Grimminger, A. Hanany, R. Kalveks, M. Sperling and Z. Zhong, Magnetic Lattices for Orthosymplectic Quivers, JHEP 12 (2020) 092 [arXiv:2007.04667] [INSPIRE].

[51] A. Bourget, S. Giacomelli, J.F. Grimminger, A. Hanany, M. Sperling and Z. Zhong, S-fold magnetic quivers, JHEP 02 (2021) 054 [arXiv: 2010.05889] [INSPIRE].

[52] O. Bergman and D. Rodríguez-Gómez, The Cat's Cradle: deforming the higher rank $E_{1}$ and $\tilde{E}_{1}$ theories, JHEP 02 (2021) 122 [arXiv:2011.05125] [INSPIRE].

[53] O.J. Ganor, D.R. Morrison and N. Seiberg, Branes, Calabi-Yau spaces, and toroidal compactification of the $N=1$ six-dimensional $E_{8}$ theory, Nucl. Phys. B 487 (1997) 93 [hep-th/9610251] [inSPIRE].

[54] M. Kreuzer and H. Skarke, No mirror symmetry in Landau-Ginzburg spectra!, Nucl. Phys. $B 388$ (1992) 113 [hep-th/9205004] [INSPIRE].

[55] I.C. Davenport and I.V. Melnikov, Landau-Ginzburg skeletons, JHEP 05 (2017) 050 [arXiv: 1608.04259] [INSPIRE]. 
[56] M. Del Zotto, C. Vafa and D. Xie, Geometric engineering, mirror symmetry and $6 \mathrm{~d}_{(1,0)} \rightarrow 4 \mathrm{~d}_{(\mathcal{N}=2)}$, JHEP 11 (2015) 123 [arXiv: 1504.08348] [INSPIRE].

[57] S. Giacomelli, RG flows with supersymmetry enhancement and geometric engineering, JHEP 06 (2018) 156 [arXiv:1710.06469] [INSPIRE].

[58] S. Cecotti and M. Del Zotto, Infinitely many $N=2$ SCFT with ADE flavor symmetry, JHEP 01 (2013) 191 [arXiv:1210.2886] [InSPIRE].

[59] S. Cecotti, M. Del Zotto and S. Giacomelli, More on the $N=2$ superconformal systems of type $D_{p}(G)$, JHEP 04 (2013) 153 [arXiv:1303.3149] [INSPIRE].

[60] M. Del Zotto, I. García Etxebarria and S.S. Hosseini, Higher form symmetries of Argyres-Douglas theories, JHEP 10 (2020) 056 [arXiv: 2007.15603] [INSPIRE].

[61] D. Gaiotto and E. Witten, S-duality of Boundary Conditions In $N=4$ Super Yang-Mills Theory, Adv. Theor. Math. Phys. 13 (2009) 721 [arXiv:0807.3720] [InSPIRE].

[62] B. Assel and S. Cremonesi, The Infrared Physics of Bad Theories, SciPost Phys. 3 (2017) 024 [arXiv: 1707.03403] [INSPIRE].

[63] F. Benini, S. Benvenuti and Y. Tachikawa, Webs of five-branes and $N=2$ superconformal field theories, JHEP 09 (2009) 052 [arXiv:0906. 0359] [INSPIRE].

[64] D. Gaiotto, $N=2$ dualities, JHEP 08 (2012) 034 [arXiv:0904.2715] [InSPIRE].

[65] O. Chacaltana and J. Distler, Tinkertoys for Gaiotto Duality, JHEP 11 (2010) 099 [arXiv: 1008.5203] [INSPIRE].

[66] D. Gaiotto and S.S. Razamat, Exceptional Indices, JHEP 05 (2012) 145 [arXiv:1203.5517] [INSPIRE].

[67] S. Cecotti, A. Neitzke and C. Vafa, R-Twisting and 4d/2d Correspondences, arXiv: 1006.3435 [INSPIRE].

[68] P.C. Argyres and N. Seiberg, S-duality in $N=2$ supersymmetric gauge theories, JHEP 12 (2007) 088 [arXiv: 0711.0054] [INSPIRE].

[69] M. Buican, S. Giacomelli, T. Nishinaka and C. Papageorgakis, Argyres-Douglas Theories and S-duality, JHEP 02 (2015) 185 [arXiv:1411.6026] [INSPIRE].

[70] M. Buican and T. Nishinaka, Conformal Manifolds in Four Dimensions and Chiral Algebras, J. Phys. A 49 (2016) 465401 [arXiv:1603.00887] [INSPIRE].

[71] M. Buican, Z. Laczko and T. Nishinaka, $\mathcal{N}=2$ S-duality revisited, JHEP 09 (2017) 087 [arXiv:1706.03797] [INSPIRE].

[72] N. Nekrasov and V. Pestun, Seiberg-Witten geometry of four dimensional $N=2$ quiver gauge theories, arXiv:1211.2240 [INSPIRE].

[73] A.D. Shapere and C. Vafa, BPS structure of Argyres-Douglas superconformal theories, hep-th/9910182 [INSPIRE].

[74] D. Xie and S.-T. Yau, $4 d N=2$ SCFT and singularity theory Part I: Classification, arXiv:1510.01324 [INSPIRE].

[75] A.D. Shapere and Y. Tachikawa, Central charges of $N=2$ superconformal field theories in four dimensions, JHEP 09 (2008) 109 [arXiv:0804.1957] [INSPIRE].

[76] S. Benvenuti and S. Giacomelli, Lagrangians for generalized Argyres-Douglas theories, JHEP 10 (2017) 106 [arXiv:1707.05113] [INSPIRE]. 
[77] S. Benvenuti and S. Giacomelli, Abelianization and sequential confinement in $2+1$ dimensions, JHEP 10 (2017) 173 [arXiv:1706.04949] [INSPIRE].

[78] S. Giacomelli, N. Mekareeya and M. Sacchi, New aspects of Argyres-Douglas theories and their dimensional reduction, JHEP 03 (2021) 242 [arXiv: 2012.12852] [INSPIRE].

[79] D. Xie and S.-T. Yau, New $N=2$ dualities, arXiv:1602.03529 [InSPIRE].

[80] D. Xie, General Argyres-Douglas Theory, JHEP 01 (2013) 100 [arXiv:1204.2270] [INSPIRE].

[81] P.C. Argyres, K. Maruyoshi and Y. Tachikawa, Quantum Higgs branches of isolated $N=2$ superconformal field theories, JHEP 10 (2012) 054 [arXiv: 1206.4700] [INSPIRE].

[82] M. Buican and T. Nishinaka, Argyres-Douglas theories, $S^{1}$ reductions, and topological symmetries, J. Phys. A 49 (2016) 045401 [arXiv: 1505.06205] [INSPIRE].

[83] M. Buican and T. Nishinaka, On the superconformal index of Argyres-Douglas theories, J. Phys. A 49 (2016) 015401 [arXiv: 1505.05884] [INSPIRE].

[84] M. Dedushenko and Y. Wang, $4 d / 2 d \rightarrow 3 d / 1 d:$ A song of protected operator algebras, arXiv: 1912.01006 [INSPIRE].

[85] S.S.-T. Yau and Y. Yu, Classification of 3-dimensional isolated rational hypersurface singularities with $C^{*}$-action, Rocky Mt. J. Math. 35 (2005) 1795 [math.AG/0303302] [INSPIRE].

[86] S. Katz, P. Mayr and C. Vafa, Mirror symmetry and exact solution of $4 D N=2$ gauge theories: 1, Adv. Theor. Math. Phys. 1 (1998) 53 [hep-th/9706110] [INSPIRE].

[87] O. Aharony, N. Seiberg and Y. Tachikawa, Reading between the lines of four-dimensional gauge theories, JHEP 08 (2013) 115 [arXiv: 1305.0318] [INSPIRE].

[88] D. Gaiotto, A. Kapustin, N. Seiberg and B. Willett, Generalized Global Symmetries, JHEP 02 (2015) 172 [arXiv: 1412.5148] [INSPIRE].

[89] A. Kapustin, Wilson-'t Hooft operators in four-dimensional gauge theories and S-duality, Phys. Rev. D 74 (2006) 025005 [hep-th/0501015] [INSPIRE].

[90] S. Cremonesi, A. Hanany and A. Zaffaroni, Monopole operators and Hilbert series of Coulomb branches of $3 d \mathcal{N}=4$ gauge theories, JHEP 01 (2014) 005 [arXiv:1309.2657] [INSPIRE].

[91] A. Hanany and R. Kalveks, Quiver Theories for Moduli Spaces of Classical Group Nilpotent Orbits, JHEP 06 (2016) 130 [arXiv: 1601.04020] [INSPIRE].

[92] J.A. Minahan and D. Nemeschansky, An $N=2$ superconformal fixed point with $E_{6}$ global symmetry, Nucl. Phys. B 482 (1996) 142 [hep-th/9608047] [InSPIRE].

[93] J.A. Minahan and D. Nemeschansky, Superconformal fixed points with $E_{n}$ global symmetry, Nucl. Phys. B 489 (1997) 24 [hep-th/9610076] [InSPIRE].

[94] I. Yaakov, Redeeming Bad Theories, JHEP 11 (2013) 189 [arXiv:1303.2769] [INSPIRE].

[95] A. Dey and P. Koroteev, Good IR Duals of Bad Quiver Theories, JHEP 05 (2018) 114 [arXiv: 1712.06068] [INSPIRE].

[96] U. Lindström, M. Roček and R. von Unge, HyperKähler quotients and algebraic curves, JHEP 01 (2000) 022 [hep-th/9908082] [INSPIRE]. 
[97] S. Cabrera and A. Hanany, Quiver Subtractions, JHEP 09 (2018) 008 [arXiv:1803.11205] [INSPIRE].

[98] F. Benini, Y. Tachikawa and D. Xie, Mirrors of 3d Sicilian theories, JHEP 09 (2010) 063 [arXiv: 1007.0992] [INSPIRE].

[99] O. Aharony, A. Hanany and B. Kol, Webs of $(p, q)$ five-branes, five-dimensional field theories and grid diagrams, JHEP 01 (1998) 002 [hep-th/9710116] [INSPIRE].

[100] O. DeWolfe, A. Hanany, A. Iqbal and E. Katz, Five-branes, seven-branes and five-dimensional $E_{n}$ field theories, JHEP 03 (1999) 006 [hep-th/9902179] [INSPIRE].

[101] N.C. Leung and C. Vafa, Branes and toric geometry, Adv. Theor. Math. Phys. 2 (1998) 91 [hep-th/9711013] [INSPIRE].

[102] D. Gaiotto, G.W. Moore and Y. Tachikawa, On $6 d \mathcal{N}=(2,0)$ theory compactified on a Riemann surface with finite area, Prog. Theor. Exp. Phys. 2013 (2013) $013 \mathrm{~B} 03$ [arXiv:1110.2657] [INSPIRE].

[103] S. Cremonesi, G. Ferlito, A. Hanany and N. Mekareeya, Coulomb Branch and The Moduli Space of Instantons, JHEP 12 (2014) 103 [arXiv:1408.6835] [INSPIRE].

[104] A. Hanany and R. Kalveks, Quiver Theories and Formulae for Nilpotent Orbits of Exceptional Algebras, JHEP 11 (2017) 126 [arXiv:1709.05818] [INSPIRE].

[105] S. Cabrera, A. Hanany and A. Zajac, Minimally Unbalanced Quivers, JHEP 02 (2019) 180 [arXiv: 1810.01495] [INSPIRE].

[106] C. Lawrie and S. Schäfer-Nameki, The Tate Form on Steroids: Resolution and Higher Codimension Fibers, JHEP 04 (2013) 061 [arXiv: 1212.2949] [INSPIRE]. 